\title{
Cardiology 2010, 13th Annual Update on Pediatric Cardiovascular Disease: Abstracts
}

\section{The Value of Pediatric Autopsy in the Diagnosis of Congenital Heart Disease: A Morphologic-Echocardiographic Correlation of 33 Cases}

Felipe Templo Jr, Marissa Orillaza, Carmela Tan, Danilo Giron

\section{Section of Cardiovascular Pathology, Division of} Laboratory Medicine, Philippine Heart Center

Background: The contrasting trend in autopsy is the global decline in autopsy rates mainly due to improved imaging while proofs are continuously generated that it clearly provides clinically relevant information. The morphologic-echocardiographic correlation of the autopsy findings in pediatric patients with congenital heart disease is one of the remaining aspects in clinical medicine where autopsy is still very much valuable. Methods: This is a cross-sectional study of 33 pediatric autopsies evaluated for congenital heart disease during a 7-year period. The pre-operative 2D-echocardiographic findings are compared with the post-mortem morphologic findings. The degree of agreement between the over-all autopsy findings and 2Dechocardiographic findings is correlated by a devised grading adapted from Underwood's criteria of clinicalnecropsy audit while the strength of the agreement of the individual isolated lesions is evaluated by kappa coefficient. Results: Over all, only 57.6\% (19) of cases have good to high morphologic-echocardiographic agreement, $12.1 \%$ (4) have some agreement and $30.3 \%$ (10) with minor to major disagreement. $9.1 \%$ (3) of cases with disagreement have significant clinical implications. For the individual lesions, all cases of Pulmonary Valve Atresia and Total Anomalous Pulmonary Venous Return have been detected preoperatively by 2D-echocardiography while all cases of Coarctation of Aorta and Persistent Left Superior Vena Cava were all missed pre-operatively and uncovered only by autopsy. The accuracy of 2D-echocardiography in the diagnosis of congenital heart disease only has $67 \%$ sensitivity, $54.8 \%$ specificity, $86.27 \%$ positive predictive value and $28.3 \%$ negative predictive value. Conclusion: There is relatively good correlation between the 2D-echocardiographic studies and morphologic findings in autopsies of pediatric patients with congenital heart disease. Missed individual lesions and discrepant diagnoses with clinical implications however still stand as problems. Thus, the autopsy should remain as an important learning and quality assurance tool in the future diagnosis and management of patients with congenital heart disease.

\section{Assessment of Ventricular Function After Percutaneous Pulmonary Valve Implantation}

Nasser Moiduddin, Karen M. Texter, John

P. Kovalchin, John P. Cheatham, Joanne L. Chisolm, Bethany L. Hashiguchi, Clifford L. Cua

\section{The Heart Center, Nationwide Children's Hospital, The Obio State University}

Background: Transcatheter pulmonary valve implantation (PVI) is an emerging therapy for pulmonary valve dysfunction. Minimal data are available assessing changes in ventricular function after PVI. We hypothesize that right and left ventricular (RV, LV) function measured with speckle tracking echo, tissue Doppler imaging (TDI), and MRI would improve after PVI. Methods: Patients who met inclusion criteria of the FDA Phase 1 Feasibility Clinical Trail were consented. Patients were prospectively studied by echocardiograms and MRI at baseline and post PVI at 3 months (echocardiogram) and 6 months (MRI). Longitudinal strain and strain rate were measured by 2D speckle tracking at basal, mid, and apical portions of the RV, interventricular septum, and LV. Standard TDI annular velocity measurements were obtained. Cardiac MRI and hemodynamic data were collected. Paired t-tests were used for analysis. Results: Ten patients were analyzed; mean age was $23.8 \pm 7.7 \mathrm{yrs}$ and mean weight was $73.0 \pm 22.1 \mathrm{~kg}$. Diagnoses included 9 patients with tetralogy of Fallot and 1 patient with complex LV outflow obstruction requiring a Ross and RV-pulmonary artery conduit. Indication for PVI was pulmonary regurgitation in 6 patients, stenosis in 2 patients, and stenosis/ regurgitation in 2 patients. After PVI, RV systolic pressure decreased $(67.3 \pm 21.3$ to $46.1 \pm 13.3 \mathrm{mmHg}$, $\mathrm{p}<0.0002$ ), and RV to pulmonary artery pressure gradient decreased $(30.3 \pm 20.6$ to $10.2 \pm 7.0 \mathrm{mmHg}$, 
$\mathrm{p}=0.002)$. By MRI RV end diastolic volume (EDV) decreased $(85.8 \pm 21.4$ to $66 \pm 15.3 \mathrm{ml} / \mathrm{m} 2, \mathrm{p}=0.01)$, while $\operatorname{RVEF}(55.5 \pm 11.7$ to $52.3 \pm 14.4 \%$ ) and LVEF $(57.2 \pm 8.1$ to $61.5 \pm 14.6 \%)$ did not meaningfully change. Echocardiographic TDI RV $(0.5 \pm 0.1$ to $0.4 \pm 0.1, p=0.045)$ and $\operatorname{LV}(0.5 \pm 0.1$ to $0.4 \pm$ $0.1, \mathrm{p}=0.008)$ Tei index improved after PVI. No other TDI measurements significantly changed. There were no significant changes in longitudinal segmental or global strain or strain rate measurements in the RV or LV. Conclusion: RVEDV and RV and LV Tei indexes showed improvement over short-term follow-up after PVI. All other non-invasive measurements showed no significant changes. Additional longer-term investigations of systolic and diastolic indices with larger sample sizes are needed to better characterize changes in ventricular function post PVI.

\section{Pulmonary Artery Development After the Norwood Operation: Comparison of Blalock-Taussig Shunt and Right Ventricle-to-Pulmonary Artery Conduit}

\author{
Remi Kowalski, Christian P. Brizard, \\ Michael Cheung, Yves D'Udekem, \\ Igor Konstantinov
}

\section{Royal Children's Hospital, Melbourne, Australia}

Background: The Norwood operation for hypoplastic left heart syndrome is associated with significant morbidity and mortality. Several retrospective studies have suggested that right ventricle-topulmonary artery (RV-PA) conduit is associated with lower post-operative mortality and improved pulmonary artery growth compared with modified Blalock-Taussig (BT) shunt. There is scant data comparing pulmonary artery growth prior to the bidirectional cavopulmonary shunt (BCPS) in these two groups. Methods: The medical records and imaging of post-Norwood infants who survived to BCPS at our hospital between January 1999 and August 2009 were reviewed. Patients were included in the study if there was adequate pre-BCPS cardiac catheterization, MRI or CT angiographic data of pulmonary artery anatomy for analysis. Results: There were 48 patients in the shunt group and 28 in the RV-PA conduit group who met the study criteria. The pulmonary artery index (Nakata index) was $194+/-30 \mathrm{~mm} 2 / \mathrm{m} 2$ in the RV-PA conduit group and $170+/-23 \mathrm{~mm} 2 / \mathrm{m} 2$ in the BT shunt group $(p=0.09)$. The RV-PA conduit patients had uniformly similar right and left pulmonary artery (LPA) diameters. The BT shunt patients had significantly smaller left than right pulmonary arteries $(4.8+1$ $-0.4 \mathrm{~mm}$ vs. $6.0+/-0.4 \mathrm{~mm}, \mathrm{p}=<0.001)$. The
LPA diameters of the shunt group were significantly smaller than those of the RV-PA conduit group $(4.8+/-0.4 \mathrm{~mm}$ vs. $5.7+/-0.7 \mathrm{~mm}, \mathrm{p}=0.01)$. There was no significant difference between the groups in terms of subsequent surgical pulmonary artery augmentation $(5 / 28$ vs. $3 / 48, \mathrm{p}=0.12)$ or tricuspid valve repair $(1 / 28$ vs. $6 / 48, \mathrm{p}=0.19)$. There was no difference in age at Norwood operation $(3.5+/-0.7$ days vs. $4.3+/-1.5$ days, $\mathrm{p}=0.18)$, timing of the pre-BCPS study $(97+/-11$ days vs. $87+/-5$ days, $\mathrm{p}=0.10)$ nor body surface area at the time of study $(0.29+/-0.01 \mathrm{~m} 2$ in both groups, $\mathrm{p}=0.46$ ). Conclusion: There is no significant difference in global pre-BCPS pulmonary artery dimensions as assessed by the Nakata index in post-Norwood patients treated with BT shunt compared with RV-PA conduit. Although the BT shunt group experienced less uniform growth of the pulmonary arteries, this was not associated with a significantly increased incidence of surgical pulmonary artery augmentation.

\section{Coronary Artery Dilatation in Toxic Shock-like Syndrome: The Kawasaki Disease Shock Syndrome}

Deane Yim, David Burgner, James Ramsay, Darshan Kothari

\section{Children's Cardiac Centre, Princess Margaret Hospital for Children, Perth, Australia}

Background: Kawasaki disease (KD) is an acute systemic vasculitis of infants and children causing coronary artery abnormalities, which may have lifelong sequelae in up to $25 \%$ of untreated patients. KD has clinical features similar to toxic shock syndrome (TSS), but they are generally considered distinct phenotypes; shock is not a feature of KD and coronary artery dilatation is not evident in TSS. However there is increasing recognition of a toxic shock-like illness with features of KD - the 'Kawasaki disease shock syndrome' - characterized by myocardial dysfunction, early onset and more severe coronary artery involvement and poor response to standard therapy. We describe two older children who satisfied diagnostic criteria for TSS and were subsequently diagnosed with $\mathrm{KD}$ when coronary artery abnormalities were found on echocardiography. Methods: Case 1 discusses the presentation of a previously well 10-year-old boy.

Case 2 discusses the presentation of a 12 -year-old boy.

The two cases presented within 5 weeks to different outer metropolitan hospitals; we could identify no other epidemiological relationships between them. Results: Both cases presented with a toxic shock-like illness requiring intensive care 
admission, aggressive resuscitation and inotropic support. They were subsequently diagnosed with Kawasaki Disease with significant ventricular dysfunction and coronary artery dilatation. The patients responded to high dose intravenous immunoglobulin and aspirin, with improvement of global function over the course of their admission. Conclusion: Kawasaki Disease (KD) misdiagnosed as toxic shock syndrome (TSS) may result in failure to assess for coronary artery (CA) damage, leading to potentially life-threatening sequelae. These cases highlight that children with KD can present with a TSS-like illness. KD should be considered in all children with TSS and echocardiography should be performed to look for CA involvement. Aggressive treatment is warranted, as long-term outcomes in untreated $\mathrm{KD}$ patients are poor. It is unknown whether CA dilatation occurs in adult TSS patients, but assessment of the CA in these patients may also be warranted.

\section{ACE Inhibitors and the Risk of Acute Kidney Injury in Infants and Children Following Cardiac Surgery}

Christina Phelps, Jennifer Eshelman, Eduardo da Cruz, Zhaoxing Pan, Jon Kaufman

\section{The Children's Hospital, Denver}

Background: Children with congenital heart disease who undergo cardiac surgery are vulnerable to acute kidney injury (AKI). AKI may result from a combination of low cardiac output and poor systemic perfusion, the effects of cardio-pulmonary bypass (CPB), or the administration of nephrotoxic medications. This study sought to evaluate the risk of developing AKI in neonates and children undergoing cardiac surgery. Methods: An IRB approved retrospective review was conducted on patients admitted to The Children's Hospital Cardiac Intensive Care Unit (CICU) following cardiac surgery from March 2007-September 2008. All patients $<2$ years of age who received furosemide alone or in combination with ACE inhibition (ACEi) were included. Data collected included: age, type of surgical repair or palliation, CPB time, cross-clamp time, circulatory arrest time, the initial and peak serum creatinine and BUN, the peak daily furosemide dose, and the timeline of ACEi initiation, peak dose, and addition of other nephrotoxic medications. Creatinine clearance $(\mathrm{CrCl})$ was calculated by the modified Schwartz criteria and the patient's maximal degree of AKI was classified by pRIFLE. A p-value of $<0.05$ was considered significant. Results: A total of 315 patients were identified. Of those, $138(44 \%)$ received furosemide therapy (furosemide group) alone while
177 (56\%) received a combination of furosemide and ACE inhibitors (ACEi group). The mean age in the furosemide group (5 months) was significantly older than in the ACEi group (3.7 months; $\mathrm{p}=0.025)$. No difference was found in the initial $\mathrm{CrCl}(\mathrm{p}=0.08)$. The CPB time was statistically longer in the ACEi group (118 minutes) versus the furosemide group (100 minutes; $\mathrm{p}=0.038)$. The cross-clamp time was also different between the two groups (66.5 minutes vs. 54.4 minutes respectively; $p=0.034)$. ACEi group had a lower post-operative $\mathrm{CrCl}(54.4 \mathrm{ml} /$ $\mathrm{min} / 1.73 \mathrm{~m} 2)$ than the furosemide group $(64.3 \mathrm{ml} /$ $\min / 1.73 \mathrm{~m} 2 ; \mathrm{p}=0.01)$ and a higher incidence of pRIFLEmax score of "F" (32.2\% vs. $21 \%, \mathrm{OR}=$ $1.78, \mathrm{p}=0.027)$. More patients in the ACEi group (45\%) developed AKI in the pRIFLE categories of either injury ("I") or "F" than patients in the furosemide group $(33 \%, \mathrm{OR}=1.6, \mathrm{p}=0.042)$. Despite more severe AKI, there was no mortality in the ACEi group in contrast to $5.8 \%$ mortality in the furosemide group. Longer cross clamp time, circulatory arrest, and the use of gentamicin were associated with an increased risk of renal failure while older age and the use of ketorolac were linked with a lower risk. After adjusting for these variables there was no difference $(\mathrm{OR}=1.000, \mathrm{p}=0.9994)$ in the incidence of AKI in patients receiving ACEi. Conclusion: ACEi are frequently used in the post-operative management of young children with congenital heart disease. Almost half of the patients receiving ACEi developed AKI with a pRIFLE score of "I" or "F". Despite an increased incidence of pRIFLEmax score of " $F$ " in patients receiving ACEi in the univariate analysis, this group did not experience an increase in mortality. The use of ACEi is not associated with AKI when the analysis is adjusted for risk factors. Finally, in contrast to other reports, ketorolac was not associated with an increased risk for developing AKI.

\section{Post-operative Electrocardiogram ST and $\mathrm{T}$ Wave Changes in Pediatric Congenital Heart Disease Undergoing Patent Ductus Arteriosus Stenting and Bilateral Pulmonary Artery Banding}

Abigail Fischer, Mark Galantowicz, John Cheatham, Beth Hashiguchi, Lawrence Schwartz, David Chan

\section{The Heart Center, Nationwide Children's Hospital, The Obio State University}

Background: The management of neonates with hypoplastic left heart syndrome (HLHS) continues to be challenging. The hybrid procedure with PDA stenting and bilateral branch pulmonary artery bands (Hybrid) is evolving as an acceptable palliative 
approach in infants with HLHS. The Hybrid does result in unique physiologic/anatomic hemodynamic concerns including coronary perfusion. We have observed EKG changes in the management of our growing experience in this population. This retrospective study sought to determine the incidence of ST-T wave changes (STC) in the early post-operative course after Hybrid. We hypothesized that STC were associated with depressed ventricular wall motion, need for early aortic arch intervention and decreased success in transitioning to stage II palliative surgery. Methods: Our cohort included patients who underwent the hybrid and who had pre and post Hybrid EKGs (within 24 hours). Demographics, echocardiographic data, clinical course, and EKG's were collected. The EKG's were analyzed by one of the authors in blinded fashion. STC was defined as $>2 \mathrm{~mm}$ depression or elevation. Pearson's Correlation and t-test were used with $\mathrm{P}<0.05$ acceptable as significant. Results: 56 patients were included and $11(19.6 \%)$ had STC postoperatively. There was no correlation between those with STC and depressed right ventricular function post-operatively. Only 2 of the STC subgroup required arch intervention. There was also no statistical significance between STC and aortic atresia. There were no differences between those with new STC and those without, in regards to needing arch intervention or proceeding to stage II palliation. Conclusion: Our study shows that significant number of patients do develop new STC in the postoperative period. These early postoperative changes did not correlate with increased morbidity. Furthermore the subgroup with STC had equal successful survival to stage II palliation. We speculate that Hybrid is associated with evolving physiology that may influence coronary perfusion. Future studies to measure other indices of ischemia and longer follow up are needed to understand the significance of these observations.

\section{Prevalence of Sinus Tachycardia in Duchene Muscular Dystrophy}

Kathi Kinnett, Richard Ittenbach, Jeanne James, Linda Cripe

\section{Cincinnati Children's Hospital Medical Center}

Background: Duchene muscular dystrophy (DMD) is a progressive myopathy resulting from mutations in the dystrophin gene. Myocardial dysfunction as well as dysrhythmias is a frequent cause of death. Sinus tachycardia has been reported in the DMD patient though the natural history of heart rate elevation in these patients has been poorly defined. The objective of this study was twofold: first, to estimate heart rate values for selected samples of boys with
DMD by age group, and second, to compare heart rate values for selected subgroups of DMD patients with established population averages. Methods: Subjects were retrospectively identified in the Cincinnati Children's Hospital Comprehensive Neuromuscular Care Center clinical database. From 1993-2009, 365 DMD males were identified and had 1,111 ECG's performed. Data were analyzed using a two-sample t-test for each of six age groups. Results are shown in Table 1 . There were statistically significant differences between age group mean heart rate estimates and population normals for the three oldest groups (8-11 yrs, $12-15$ yrs, $16+$ yrs $) \quad(\mathrm{p}<0.001)$. Results: Table 1. Comparison of Normal Heart Rates to DMD Patients.

\begin{tabular}{llll}
\hline Age (years) & Normal & DMD & N of DMD ECGs \\
\hline $1-2$ & $119(89-151)$ & $116(98-166)$ & 19 \\
$3-4$ & $108(73-137)$ & $108(89-37)$ & 90 \\
$5-7$ & $100(65-133)$ & $99(62-135)$ & 253 \\
$8-11$ & $91(62-130)$ & $99(62-147)$ & 353 \\
$12-15$ & $85(60-119)$ & $100(50-181)$ & 225 \\
$\geqslant 16$ & $70(60-100)$ & $101(58-161)$ & 171 \\
\hline
\end{tabular}

Conclusion: Heart rate increases with age in the DMD patient. Average heart rate was not increased in the younger patient less than 7 years of age and should prompt additional investigation when noted. In addition, sinus tachycardia is common in the older DMD patient. Additional studies are needed to more clearly establish the link between heart rate and dystrophin deficiency.

\section{The Utility of a 15-Lead Electrocardiogram in Determining the Laterality of an Accessory Pathway in Wolff-Parkinson- White Syndrome}

Peter Aziz, Tammy Wieand, Jamie Ganley, Maully Shah

\section{The Cardiac Center, The Children's Hospital of Philadelphia}

Background: Several algorithms have been developed to describe the location of accessory pathways (AP) in Wolff-Parkinson-White (WPW). Standard 12 lead ECG assessments have been utilized to predict AP location in published algorithms. In ECGs showing sub-maximal ventricular pre-excitation, conventional AP algorithms may not be applicable. The objective of this study is to validate a simple algorithm for determining AP laterality (right vs. left) using the addition of right pre-cordial leads $(\mathrm{V} 3 \mathrm{R}, \mathrm{V} 4 \mathrm{R})$ to the standard ECG. Methods: A retrospective study of 
patients with structurally normal hearts who had undergone successful catheter ablation (CA) for WPW from 1/2002-1/2008 was performed. Patients with multiple AP or patients with congenital heart disease were eliminated from the study. Pre-ablation 15-lead ECGs of all patients were assessed. Delta wave polarity (first $40 \mathrm{~ms}$ of QRS), QRS duration, PR duration and QRS polarity in leads V3R and V4R were specifically evaluated by two readers. Readers were blinded to the electronic ECG read and to pathway laterality. An algorithm was then tested using positive QRS polarity in leads V3R and $\mathrm{V} 4 \mathrm{R}$ as predictive of a left-sided pathway and a negative QRS polarity in leads V3R and V4R as predictive of a right-sided pathway. Results: 185 patients fulfilled criteria for study. Right-sided AP was confirmed during CA in 107 patients (58\%) and left-sided AP in 78 patients (42\%). Using our algorithm, the positive predictive value of a rightsided AP was 88\% (105/119) and 89\% (105/118) for both readers. The positive predictive value of a left-sided AP was $97 \%(60 / 62)$ and $98 \%(60 / 61)$ for both readers. In addition, 4 to 6 patients (depending on reader interpretation) had equivocal polarities in leads V3R and V4R. Of these patients, 3/4 or $5 / 6$ had left-sided pathways. Delta wave polarity in V3R and V4R was not predictive. Conclusion: A simple algorithm for determining laterality of an AP in WPW can be obtained by adding right precordial leads to a standard ECG prior to catheter ablation. This information may help plan mapping and ablation strategies as well as counsel families regarding procedural outcome and risk.

\section{Optimizing Surgical Infection Prevention in Pediatric Cardiovascular Surgery Patients}

Sandra Staveski, K. Mathews, S. Trotter, J. Buckle, R. Simon

\section{Lucile Packard Children's Hospital at Stanford}

Background: The consequences of surgical site infections (SSIs) can be significant ranging from discharge delays to mediastinitis. Meticulous interdisciplinary care and vigilance are the keys to reducing SSIs. A cluster of SSIs led us to critically evaluate our practice. We outline our systemic approach in reducing SSIs. Methods: An interdisciplinary quality improvement initiative was developed to optimize care delivery for our patients. We surveyed best practices, performed a literature search, and benchmarked industry standards for pediatric cardiovascular surgery SSI rates. We then traced patient movement through our care continuum and identified areas for practice improvements. We collaboratively developed a new interdisciplinary surgical infection prevention standard for pediatric cardiovascular patients utilizing the Children's Healthcare Corporation of America (CHCA) surgical infection prevention (SIP) bundle. The new care standard included: 1) implementation of age-appropriate antimicrobial pre-operative baths; 2) implementation of a pre-operative order set; 3) updating our pre-operative checklist; 4) development and distribution of a letter to our families; 5) implementation of clippers for hair removal; 6) improved compliance with prophylactic antibiotic choice, timing, redosing during prolonged surgical cases, and discontinuation post-operatively; 7) implementation of Chloroprep operative scrub for specific patient populations in place of Betadine; 8) post-operative nursing wound care education; and 9) use of disposable ECG leads. The institutional infection control department, quality department, and patient safety program tracked compliance with the practice changes and SSI rates. Data were reviewed by the team routinely. Results: Our baseline SSI rate was $3.8 \%$. During the cluster, increased to $9.2 \%$. After SIP implementation, compliance with best practice increased from $40 \%$ to $100 \%$ and our SSI rate has reduced to $2.8 \%$. Conclusion: This patient safety initiative has improved our patient outcomes and enhanced collaborative interdisciplinary practice.

\section{Echocardiographic Parameters that Predict Outcome in Aortic Atresia Patients Undergoing Comprehensive Stage II Procedure}

Brian Birnbaum, Glen Berger, Bernadette Fenstermaker, Daniel G. Rowland, Bethany Boettner, Vince Olshove, Mark Galantowicz, John P. Cheatham, Clifford L. Cua

\section{The Heart Center, Nationwide Children's Hospital, The Obio State University}

Background: The hybrid procedure is one option for palliating patients with hypoplastic left heart syndrome. The next surgical procedure in this pathway consists of stent removal, pda ligation, pulmonary artery band removal, aortic arch reconstruction, and a primary cavopulmonary connection. There is limited information regarding the correlation of echocardiographic findings prior to this major surgery. The goal of this study was to determine if echocardiographic parameters correlate with post-operative variables in patients with aortic atresia undergoing the comprehensive stage II procedure. Methods: Retrospective chart review on all patients with aortic atresia, who underwent the comprehensive stage II procedure from January 2002 to December 2008, was performed. 
Patients were excluded if they did not have the diagnosis of aortic atresia, if they did not undergo the comprehensive stage II procedure, or if there was not an echocardiogram performed within one month of the procedure. Echocardiographic indices evaluated included end systolic and diastolic RV area, change in $\mathrm{RV}$ area, eccentricity index, tricuspid inflow velocities, antegrade and retrograde volume-time integral through the stented ductus and calculated cardiac output. Peri-operative surgical indices evaluated included pre- and post-bypass lactate levels, maximum lactate level, length of stay in the intensive care unit and in the hospital, length of intubation, maximum inotrope score, duration of inotropes, chest tube drainage, urine output and fluid balance for post operative days one through five, use of ECMO, renal or hepatic insufficiency, dialysis, and in-hospital death. Pairwise Pearson's correlation tests were used to analyze the associations between continuous measures. Variables that were not normally distributed were logged to normalize the distribution before doing correlation tests. $\mathrm{P}$ value $<0.05$ was considered significant. Results: Thirty-four patients met inclusion criteria. Age at comprehensive stage II procedure was $0.45+0.13$ years and body surface area was $0.30+$ $0.03 \mathrm{~m} 2$. RV \% change was $45+10 \%$, eccentricity index was $0.54+0.17$, estimated cardiac output was $8.7+6.69 \mathrm{~L} / \mathrm{min} / \mathrm{m} 2$ and estimated effective cardiac output was $5.8+4.24 \mathrm{~L} / \mathrm{min} / \mathrm{m} 2$. Retrograde PDA VTI correlated with $\log$ pre-bypass lactate and maximum lactate $(r=0.53,0.44)$. PDA regurgitant fraction correlated with log post-bypass lactate, length of intubation, and urine output on day four $(\mathrm{r}=0.39$, $0.46,-0.37)$. RV \% change correlated with log pre-bypass lactate, and urine output on days four and five $(r=-0.38,0.43,0.54)$. No echocardiographic parameter predicted renal or liver insufficiency, dialysis, open sternum, ECMO use, or hospital death. Conclusion: Retrograde PDA VTI and RV \% change correlated with some peri-operative variables though no echocardiographic parameter was associated with any major morbidities or mortality. Newer echocardiographic techniques with a larger patient population are needed to better define echocardiographic parameters that may be predictive of comprehensive stage II outcomes.

\section{Cardiogenetics: The Past, Present and Future}

Esma Paljevic

\section{Children's Hospital at Montefiore Medical Center}

Background: According to the American Heart Association sudden cardiac death (SCD) is a sudden, unexpected death caused by loss of heart function. Sudden death occurs most frequently in adults in their mid-30s to mid- $40 \mathrm{~s}$, and affects men twice as often as it does women (AHA). SCD is rare in children, affecting only 1 to 2 per 100,000 children each year. The most common cause of sudden cardiac death in the young has been identified as an arrhythmogenic event, such as ventricular fibrillation resulting from cardiac channelopathies. Sudden cardiac death in one family member increases the chance of this occurring in other family members, including children. Research regarding families who have experienced sudden death indicates that these families require a great deal of social support. Typically, relatives of young sudden death victims are referred to cardiologists for a history and physical. Until quite recently it was one referral after another. The families were referred to various sub-specialists to be seen in different clinics on different days as well as a social worker for family support. Methods: The team was put together by discussions and meetings of the specialties. The team includes: a pediatric and a separate adult cardiologist, geneticist, nurse practitioner, genetic counselor, social worker and research fellows. The nurse practitioner coordinates the clinic and speaks to the families before, during and after the clinic. She presents the cases and does all the follow-up on the families. The team discusses the role of genetic testing, the implications of the results and many other very interesting issues. This new testing allows health care providers to identify those patients in families who had a family member die from sudden cardiac death who are at increased risk for also experiencing sudden cardiac death in the future. Results: At present we have evaluated over twenty (20) families. The team has submitted an $\mathrm{NIH}$ grant proposal to look at the social implications of genetic testing in the families that have experienced sudden death in a family member. I am also submitting a doctoral dissertation proposal looking at anxiety and quality of life issues in the families that are referred to the Cardiogenetics Clinic. The team is starting to formally look at the implications of this clinic. Anecdotally, the families are grateful for having a better understating of the information that has been given to them regarding the true incidence of sudden death. They state that they feel more "in control" and can at least take steps to gain information whereas before this clinic they always heard "we just don't have that information". Conclusion: In the past families who had a member suffer sudden cardiac death were told the potential for them to have the same condition was unknown. With new DNA testing health care providers can offer families the ability to identify 
those members 'at risk' for suffering sudden cardiac death. The purpose of a Cardiogenetics clinic is to provide these families with appropriate clinical screening, testing (when indicated) and counseling. This clinic aims to identify and to educate family members and hopefully address their anxiety and stress. The difficulty arises in that the actual potential for sudden death cannot be predicted or prevented. This is were the Cardiogenetics Team at Montefiore Children's Hospital would like to make a difference.

\section{Defining Clinically Valid Thresholds for Body Fatness Related to Dyslipidemia in Adolescents}

Ashish Sureka, Tom Robinson

\section{Lucille Packard Children's Hospital, Stanford University}

Background: Many measures of obesity have been proposed, including body mass index (BMI), BMI percentile, waist circumference (WC), triceps skinfold thickness (TST), and waist-to-height ratio (WTHR). Methods: Anthropometric measures were analyzed in their ability to predict dyslipidemia (total cholesterol (TC) $200 \mathrm{mg} / \mathrm{dL}$ or above, LDL $130 \mathrm{mg} / \mathrm{dL}$ or above, HDL $35 \mathrm{mg} / \mathrm{dL}$ or below, or triglycerides (TRIG) $150 \mathrm{mg} / \mathrm{dL}$ or above) in 413 preadolescent girls. Using ROC analysis, optimal cutpoints for BMI, percentile, WC, TST and WTHR were determined; these cutpoints were then compared to current screening practices (BMI of 85th percentile and BMI of 95th percentile). Results: WTHR had a better AUC than BMI percentile for predicting abnormal TC, LDL, and TRIG, and a better odds ratio (OR) than BMI percentile in all dyslipidemia categories. Ethnicity, age, and sexual maturity did not affect the cutpoints. Conclusion: WTHR is a useful screening tool for dyslipidemia in the pediatric population and, in some respects, superior to BMI and BMI percentile. Ethnicity and age did not have a significant effect on the cutpoints to predict dyslipidemia.

\section{Angiotensin II Receptor Blocker Slows the Rate of Progression of Aortic Root Dilatation in Marfan Syndrome}

Praveen Kumar, Andrew Griffin, Catherine Harris, Huda Elshershari

\section{University of Illinois at Chicago}

Background: Marfan syndrome is an autosomal dominant connective tissue disorder in which abnormalities occur in the cardiovascular, ocular and musculoskeletal systems. Majority of mutations occur in FBN1 gene located at chromosome 15q21.1. However 10\% mutations occur in Transforming growth factorbeta-receptor 2 (TGFBR2) and TGFBR1 gene. The fibrillin -1 is an important constituent of connective tissues. The histopathology of aortic tissues shows fragmentation of elastic lamellae and fibrosis. The exact molecular mechanism is not understood but one of the proposed mechanisms is increased bioavailability of Transforming growth factor-beta (TGF- $\beta$ ). Recent research from mouse models of Marfan syndrome demonstrates aortic root enlargement due to excessive signaling by TGF- $\beta$. Angiotensin II type 1 receptor blocker (Losartan) is a TGF- $\beta$ antagonist. We present 2 cases of Marfan syndrome with aortic root dilatation stabilized on losartan treatment. Methods: Case 1: This infant was born to a Hispanic family where father was diagnosed with Marfan syndrome for many years. He has had aortic valve replacement and multiple eye surgeries. The mother and her other 2 children from other partner are healthy. The infant was born at term by spontaneous vaginal delivery with a birth weight of 3200 gram (25th percentile) and a length of $51 \mathrm{~cm}(>50 \mathrm{th}$ percentile). Physical examination revealed long and protruding ears, micrognathia, high palate, arachnodactyly and undescended testicles. No heart murmur. Echocardiogram at 1 month of age showed aortic root dilatation measured $1.60 \mathrm{~cm}(\mathrm{Z}=2.94)$. Molecular genetic studies showed a mutation in exon 31 of the FBN1 gene in the infant and Father. The infant is treated with losartan and propranolol. During follow up, serial echocardiograms showed stable aortic root sinus dilatation. At 9 and half months Aortic sinuses measured $1.86 \mathrm{~cm}(\mathrm{Z}=3.4)$.

Case 2: A 3 and half-year-old Hispanic male presented to ophthalmology department with chief complaint of failed school visual examination which was diagnosed as bilateral ectopia lentis. Physical examination revealed tall stature $(>95 \%)$, large ears, high arched palate and hyper extensible elbow. No heart murmur. Further genetic testing confirmed FBN1 gene mutation in the child and mother. Echocardiogram at 4 and half years showed aortic sinus dilatation of $2.45 \mathrm{~cm}(\mathrm{Z}=2.37)$ and was started on losartan. Subsequent follow up at 4 years and 10 months showed stable aortic sinus dilatation, measured as $2.63 \mathrm{~cm}(\mathrm{Z}=2.55)$. Results: Serial aortic sinus diameters of case 1

\begin{tabular}{lll}
\hline Age (Month) & Aortic Sinus $(\mathrm{cm})$ & $\mathrm{Z}$ score \\
\hline 3.5 & $1.67 *$ & 3.40 \\
6.5 & $1.77 \mu$ & 3.39 \\
9.5 & $1.86 \mu$ & 3.40 \\
\hline
\end{tabular}


Serial aortic sinus diameters of case 2

\begin{tabular}{lll}
\hline Age & Aortic Sinus $(\mathrm{cm})$ & $\mathrm{Z}$ score \\
\hline 4 year and 6 month & $2.45 *$ & 2.37 \\
4 year and 8 month & $2.55 \mu$ & 2.53 \\
4 year and 10 month & $2.63 \mu$ & 2.55 \\
\hline
\end{tabular}

*Before losratan therapy; $\mu$ after losartan therapy

Conclusion: Losartan is a new therapy for stabilizing aortic root dilatation in Marfan syndrome. We stress the importance of early recognition in order to anticipate and treat the cardiac abnormalities that are likely to arise and were detected soon after birth in the infant reported in this report. The infant (Case1) is youngest till date to receive the treatment for aortic root dilatation due to the rapidity of the progression which contributed to the decision to use in this very young age. Losartan significantly slowed the rate of enlargement of the aorta in our patients.

\section{Efficacy of Car Seat Testing Following Reparative Congenital Heart Surgery: A Cross-Sectional Analysis of 152 Consecutive Neonates}

Megan Gilbert, Phillip Nevin, Velva Woodward, Jeffrey Pearl, Mitchell Cohen

\section{Phoenix Children's Hospital}

Background: Car seat challenge testing is completed for all neonates prior to discharge following congenital heart surgery by the bedside cardiac nurse for a duration equivalent to one hour or the length of transit time to their home. Failure of the test from apnea, bradycardia or hypoxemia indicates a need for further evaluation prior to discharge. A prior study reported a $4 \%$ failure rate though the sample population was substantially smaller and no follow-up testing assessed efficacy. Methods: A search of the Cardiology/CV surgery database at Phoenix Children's Hospital identified all neonates who underwent surgery from January 2006December 2008. A detailed chart review of all demographic data was obtained including evaluation of the car seat test. Following IRB approval, a questionnaire was sent to the families querying them on any events (cyanosis, apnea, seizures) that may have taken place while in their car seat following discharge. Failure to return questionnaire prompted a second mailing. For families whose neonate had an "event" while in the car seat, a phone call to understand the exact circumstances was performed. Statistical analysis was performed comparing patients who responded to the questionnaire to those who did not to assess the adequacy of the sample population; as well as analysis of single ventricle versus two-ventricle patients. Results: 152 neonates (age at surgery 10.4 \pm 8.4 days; birth weight $3.13 \pm 0.56 \mathrm{~kg}$ ) underwent heart surgery \{RACHS \#1 (5) \#2 (34) \#3 (51) \#4 (33) \#5 (1) \#6 (28)\}. All RACHS-6 patients underwent Norwood palliation. Single-ventricle physiology existed in 48 patients $(32 \%)$. The median length of stay was 11 days (range 2-152 days). Major reoperations occurred in $8(7.6 \%) 2$-ventricle patients and in $6(12.5 \%)$ single-ventricle patients. Two patients failed initial car seat testing ( 7 day old obstructed supracardiac TAPVR and 11 day old arterial switch). All other neonates were discharged having passed their initial car seat test. Follow up surveys were obtained in $38(25 \%)$ patients. There was no significant difference in the percentage of returned questionnaires between 1-ventricle and 2-ventricle patients. $29 \%$ of parents did not understand the purpose of discharge car seat testing. Five patients had perceived cyanotic episodes shortly after discharge from the hospital while in their car seat, 4 of these patients passed their initial car seat test; the 5th patient (the TAPVR) failed his initial car seat test. All of these patients were admitted to the hospital following these events. Conclusion: Car seat testing appears to be a relatively safe intervention performed by the bedside nurse prior to discharge. The observation that $1 / 3$ of families did not understand the reason the car seat test was performed supports the need for better parental education by nursing. The false positive rate for car seat testing after discharge was $10.5 \%$. While initial passing of the car seat test is reassuring it does not guarantee that the child's hemodynamics might not change and result in an event that warrants further evaluation.

\section{Children and Adolescents with Attention Deficit Hyperactivity Disorder in a Pediatric Cardiology Office}

Sudhir Mehta, Nancy Richards, Irwin Jacob

\section{Cleveland Clinic/Fairview Hospital}

Background: Despite the heightened awareness since 2006 of the cardiac controversy concerning the use of stimulant medication in children and adolescents with Attention-Deficit/Hyperactivity Disorder (ADHD), there is a paucity of information regarding any consequent change in the reasons for patient referrals to a pediatric cardiologist. Objective: To compare the relative frequency of common reasons for referral to a pediatric cardiologist before and 
after 2006 and to compare the reasons for referral of those patients with a co-morbid diagnosis of ADHD with those who did not have this co-morbid diagnosis. Additionally, to investigate predictors of heart rate in subject taking stimulants with other confounding factors like age, blood pressure, and Body Mass Index (BMI) or Waist Circumference (WC). Methods: From March 2004 to May 2009, data of 74 subjects with co-morbid ADHD who were referred for a pediatric cardiology evaluation (Group I) in a community hospital were compared with a random sample of 74 control subjects of similar age range (Group II). Results: During and after 2006, as compared to pre-2006, a higher percentage of patients were referred for cardiology evaluation who had co-morbid ADHD (53 vs. 21 or $11.6 \%$ vs. $6.9 \%, \mathrm{P}=0.0337)$. A significantly higher number of subjects in Group I vs. Group II were referred because of an abnormal ECG $(\mathrm{N}=13$ vs. $0 ; \mathrm{P}<0.0001)$. Stimulant use in 56 subjects with ADHD was the strongest predictor of heart rate in our population. Conclusion: Our preliminary observations show a relative increase of referral of patients with co-morbid ADHD to a pediatric cardiologist since 2006. Stimulants, rather than obesity, cause higher heart rates in these individuals. Usefulness of routine ECG and its possible outcome benefits require further studies.

\section{Economic Impact on Research: How Do You Optimize Your Resources to Conduct and Produce Quality Clinical Research?}

Donna Sylvester, Kathy Gilmartin

\section{The Cardiac Center, The Children's Hospital of Philadelphia}

Background: The economy is not going to turn around overnight, so it is vital to be creative and to tap into all of your available resources to conduct research. Flexibility and restructuring may be the key to your success. Having the right people in the right position, collaboration and study design are key components to a successful trial and research center. Methods: Initially make sure that those individuals in key positions are the right people for those jobs. Develop a team approach, which optimizes cross coverage. Responsibilities must be clearly defined, inculcating ownership. At the same time, the team must adopt the concept that no team member is too good to perform another team member's job. This will allow a site to increase its number of active trials, ultimately increasing enrollment and productivity. It is critical to share one's knowledge with other team members. Sharing experiences both good and bad is a forum that can be an invaluable learning tool.

Begin to evaluate systems. This cannot be a long, arduous process. Time is money. Once the failed systems are identified, determine how and if they can be rectified or is it more efficient to eliminate and restructure? You will discover systems that are not in place, which will need to be developed. Allow the team to participate in the development phase.

Developing trials with subspecialties and other disciplines will provide you with a plethora of data from many areas. The key is to be organized and systematic. It may seem to be intuitive but you will see many investigators collect multiple data points and only a small percentage will yield anything valuable. It is important to stay focused. Remember time is money and if you are spending all of your time collecting useless data you are wasting your time. It is critical to develop timelines and stick to them. Results: In the 1st 4 months -

Number of Cardiology studies in the Division: 98 Number of Studies Supported by Cardiology Core

Team (4 Coordinators): 23

MDs supported through the Core Team: 9

Data Abstraction (no consent): 402

Subjects Enrolled: 114

Active Subjects on Study (in follow-up): 72

Amendments: 18

Continuing Reviews: 9

New submissions: 3

Developed Clinical Trial Operations Manual - Study

Specific: 8

SAE Reports: 8

CRC Submissions: 2

Additional IRB Consults: 4

Protocol Consults: 4

Grant Submissions - RO1: 7

Grant Submissions - RC2: 2

Grant Submissions - U01: 2

Grant Submissions - P50: 1

Grant Submissions - T32: 2

Grant Submissions - Foundation: 2

Grant Submissions - Cardiac Center Grants: 4

Multi-center trials: 1 with 14 external sites

Conclusion: In conclusion, key personnel who are sometimes underestimated and undervalued are volunteers, students, statisticians and database personnel. Have your statistician assist with study design and review your case report forms. Students and volunteers are a tremendous resource that is greatly underutilized. Database development and management are vital. Many investigators feel this piece of the trial is unproblematic. It is a good idea to include all of the aforementioned in your budget; it will be well worth the investment. 
Coping While Caring for the Dying Child: Nurses' Experiences

Katherine Cook, Patricia Lawrence, Mary Rose Grady, Denise Norton, Kimberly Liner, Julie Jablonski, Jennifer Cioffi, Patricia Hickey, Suzanne Reidy, Jean Connor

\section{Children's Hospital Boston}

Background: The purpose of this study is to describe and understand the coping mechanisms used by nurses when caring for dying children on an inpatient acute care cardiology unit. We sought to understand the resources and support that nurses feel are necessary to maintain a healthy work environment.

There is a minimal amount of research in the area of nurse's experiences and their ability to cope while caring for a dying child. Many patients spend months in a chronically ill state on an acute care unit before moving to the intensive care unit or dying on the acute care unit. During prolonged hospitalizations of chronically ill patients on an acute care unit, many nurses develop strong relationships with patients and their families. Most studies have focused on how intensive care nurses and oncology nurses cope with loss. However, few look at the coping abilities of acute care unit nurses. Methods: Qualitative methods using focus groups were conducted with acute care nurses who had cared for a dying child after a prolonged illness within the Cardiac Unit at a large tertiary care pediatric hospital. Chronic illness was defined as any medical condition that requires long-term management of greater than six months and one or more periods of hospitalization. Nurse participants cared for at least one chronically ill child who died at Children's Hospital Boston. Informed consent was obtained. Semi-structured focus groups lasting approximately 60 minutes were scheduled during the nurses participating shift and were conducted within the inpatient Cardiac unit at Children's Hospital Boston. Data was audio taped, transcribed verbatim and categorized into domains. During the first interpretive phase, the researchers reviewed comments from the identified data source, highlighting illustrative instances in the comments, and tracking patterns and categories that appeared within and between comments. The researchers then interpreted and contextualized the data for further analysis, organization and coding. Results: Twentytwo nurses participated in focus groups prior to reaching saturation. Four main categories were identified: Disconnecting, Memories, Boundaries, and Labeling. Prior experiences or exposures were found to inform coping and behaviors used by nurses. Colleague support and institutional resources were critical to the coping and grieving process. Years of practice influenced the nurse's ability to disconnect and set boundaries. Conclusion: Many experiences were identified that impacted nursing coping behaviors and mechanisms. Coping and grieving are a critical part of the practice of nurses in a cardiac acute care unit. Colleague support and adequate resources allow for an adequate grieving process. We hope that certain categories and impacts that were identified may be used to improve nursing coping behaviors and mechanisms. It is well described in the literature that job dissatisfaction, stress and burnout result from an inadequate grieving process. Further studies may be conducted to further look at this relationship.

\section{Physical Activity and Self-Efficacy in Children with CHD: Is There a Connection}

Trenda Ray, Angela Green, Leanne WhitesideMansell, Karen Henry

\section{Arkansas Children's Hospital, University of Arkansas for Medical Sciences}

Background: Children with even the most complex congenital heart disease (CHD) commonly survive into adulthood. With the improved survival, new long-term healthcare issues are emerging including low physical activity self-efficacy which impacts not only the children's participation in physical activity, but also their quality of life. This, in combination with their underlying cardiac disease and limitations in exercise capacity, may be a mechanism that leads to an increased risk of overweight or obesity in children with CHD. These concepts and their relationships have yet to be fully explored in this population. The purpose of this study is to describe the specific relationships among the following variables in children with CHD who are 10-14 years of age: physical activity self-efficacy, physical activity habits, and body mass index (BMI). Methods: A cross-sectional study is currently underway. Participants are being recruited from the Cardiology Clinic at Arkansas Children's Hospital with a targeted sample size of 84 . Children with medically prescribed activity restrictions are excluded. Participating children complete the Previous Day Physical Activity Recall (PDPAR) Instrument, the physical activity items from the Youth Risk Behavior Survey (YRBS), the SelfEfficacy Scale (SES) Instrument (which is specific for physical activity), and an investigator developed Demographic form. Participant BMI is collected from the clinic record. Results: Forty-two children 
have been enrolled to date (average age 12 years). The sample is evenly divided between males and females and is predominantly white (83\%). Diagnostic categories of participants include: (1) mild requiring no therapy or treated without surgical intervention $(16.7 \%)$, (2) moderate requiring no therapy or surgically corrected $(21.5 \%)$, (3) surgically treated need for future surgical interventions $(40.5 \%)$, (4) complex or severe, uncorrectable or palliated $(21.4 \%)$. The average physical activity of participants, as defined by metabolic equivalent tasks (METS) was $33(\mathrm{SD}=13)$ METs per day based on the PDPAR. The average total self-efficacy score was $30.7(\mathrm{SD}=5.6)$ out of a maximum of 40 (range 15-38). Both measures were approximately 50\% lower than what is described for healthy children in the literature. The average absolute BMI was $23(\mathrm{SD}=7.8)$. Self-efficacy and physical activity were positively correlated indicating that children with higher self-efficacy had higher levels of physical activity $(\mathrm{r}(42)=0.33 ; \mathrm{p}=0.042)$. However, self-efficacy and absolute BMI were not significantly correlated $(\mathrm{r}(42)=0.004 ; \mathrm{p}=0.98)$. Conclusion: In this small sample of children with $\mathrm{CHD}$, self-efficacy and physical activity were lower than published data for healthy children. This is concerning, given the risk for overweight and obesity. Results suggest that children with CHD have similar links between self-efficacy and physical activity (positive correlation) as found in children without CHD. Therefore, it is possible that interventions that increase self-efficacy may have important effects on long-term physical health. This may be as simple as encouraging the children to participate given that verbal persuasion is key to the development of selfefficacy. While self-efficacy and absolute BMI were not correlated, this may reflect that absolute BMI rather than BMI percentile was used. This analysis is planned with the final analysis.

\section{Twenty-three Year Experience of Acute Rheumatic Fever in a Tertiary Hospital in Australia}

Deane Yim, B. Chidlow, M. Tallon, S. Woods, J. Ramsay

\section{Children's Cardiac Centre, Princess Margaret Hospital for Children, Perth, Australia}

Background: Acute rheumatic fever (ARF) is a leading cause of paediatric acquired heart disease amongst Indigenous populations of Australia. It results from an autoimmune response to a Group $A$ streptococcal infection and occurs predominantly in children aged 5-14 years. Of major significance is the development of long-term valvular heart disease. To date there has been no published data describing ARF presentations from tertiary hospitals in Australia. Princess Margaret Hospital (PMH) is the only tertiary children's hospital in Western Australia (WA), and is the centre of patient transfers from WA peripheral hospitals. The aim of our study is to describe ARF presentations to Princess Margaret Hospital in Perth, from 1986 to 2008. Methods: A retrospective review of ARF presentations to PMH between January 1986 and December 2008 was conducted. Cases were identified if they were assigned an ICD diagnosis of primary ARF or recurrence of $\mathrm{ARF}$ on hospitalisation discharge data. Only cases meeting the modified Jones criteria (1986-2005) or National Heart Foundation of Australia (NHFA) diagnostic criteria (2005-2008) for ARF were included. Results: There were 97 admissions in 72 patients had an ICD code for ARF, and 86 cases in 69 patients satisfied NHFA criteria. 96\% identified as Aboriginal and Torres Strait Islanders (ATSI) and 4\% of Caucasian descent. 87\% of cases were between 5 and 14 years of age and the median age was 10 years. $29 \%$ of cases were recurrent episodes of ARF. $46 \%$ of $\mathrm{PMH}$ admissions were from the metropolitan area, with the rest transferred from peripheral centres. There was no indication that the rates of admission were reducing over time. The presenting features included carditis or subclinical carditis in $81 \%$, polyarthritis in $40 \%$ and chorea in $35 \%$. Two patients presented with subcutaneous nodules and two presented with erythema marginatum. The average length of stay was 17 days for ARF. $10 \%$ of patients who presented with carditis required surgery. To our knowledge there has been one late death associated with rheumatic heart disease. Antibiotic prophylaxis was prescribed in $98 \%$, however compliance to prophylaxis has been poor. Over the last 10 years, only $35 \%$ of patients have been documented to be compliant. We observed that patients who were compliant to prophylaxis did not have an episode of ARF recurrence. Conclusion: ARF remains a significant health issue affecting Indigenous children. There is a striking ethic disparity and is considered to be a disease of poverty. There is no indication that rates of admission are reducing over time, which may suggest that there has been little improvement in primary and secondary preventative measures in 23 years. Non-compliance to prophylaxis and follow-up remains a major issue, and there is an unaaceptably high recurrence rate which is most certainly underestimated. Our research reflects the need for effective discharge planning and promoting transition to Aboriginal Health services involved in continuing follow-up. The WA surveillance registry 
has been established to support patients with ARF, and to implement ongoing initiatives for health promotion and education.

\section{Octreotide for the Treatment of Chylothorax in Pediatric Patients Following Cardiothoracic Surgery}

Christopher Rausch, Lindsay Caverly, Eduardo da Cruz, Jon Kaufman

\section{The Children's Hospital, Denver}

Background: The incidence of chylothorax in pediatric patients after cardiothoracic surgery is variable but has been reported to be up to $6 \%$. Treatment options vary, however despite drainage of the effusion, dietary manipulation, or bowel rest and total parenteral nutrition, successful resolution may be difficult to achieve. Many cases of chylothorax are persistent or severe enough to require surgical intervention such as thoracic duct ligation or pleurodesis. Several reports have shown varied results in the efficacy of octreotide therapy leading to resolution of chylothorax. This study sought to evaluate the efficacy of octreotide for persistent chylothorax in children following cardiothoracic surgery. Methods: An IRB approved retrospective review was conducted on patients admitted to the The Children's Hospital Cardiac Intensive Care Unit between 1998-2008. Data collected included age, sex, type of cardiac lesion and surgical procedure, postoperative day octreotide therapy was initiated, maximum drainage prior to octreotide therapy and maximal drainage during octreotide therapy, dose range of octreotide, days to resolution, and complications.

\begin{tabular}{|c|c|c|c|}
\hline & Resolved & $\begin{array}{l}\text { Did Not } \\
\text { Resolve }\end{array}$ & $\mathbf{P}$ \\
\hline $\mathrm{n}$ & 12 & 7 & \\
\hline Male & $8(67 \%)$ & $6(85 \%)$ & \\
\hline Age (m) & $\begin{array}{c}3.9 \\
(0.4-48)\end{array}$ & $\begin{array}{c}6 \\
(0.36-48)\end{array}$ & \\
\hline POD & $10(2-22)$ & $7(1-240)$ & \\
\hline $\begin{array}{l}\text { Days prior to } \\
\text { starting } \\
\text { Octreotide }\end{array}$ & $5(1-26)$ & $10(2-33)$ & 0.12 \\
\hline $\begin{array}{l}\text { Maximum } \\
\text { drainage prior to } \\
\text { Octreotide } \\
(\mathrm{mL} / \mathrm{kg} / \text { day })\end{array}$ & $42(+25)$ & $95(+79)$ & 0.05 \\
\hline $\begin{array}{l}\text { Maximum } \\
\text { drainage during } \\
\text { Octreotide } \\
(\mathrm{mL} / \mathrm{kg} / \text { day })\end{array}$ & $20(+15)$ & $73(+81)$ & 0.04 \\
\hline Days to resolution & $8(4-21)$ & $\mathrm{n} / \mathrm{a}$ & \\
\hline Side effects & 2 & 0 & \\
\hline Deceased & 0 & 2 & \\
\hline
\end{tabular}

Results: Nineteen patients were identified who underwent treatment with octreotide for chylothorax following cardiothoracic surgery. All 19 patients had undergone dietary therapy and pleural tube placement prior to starting octreotide in an attempt to treat the chylous drainage. In $63 \%(12 / 19)$ there was resolution of the chylothorax. $74 \%$ (14/19) demonstrated a decrease in the peak effusion drainage rate following initiation of octreotide, however in 2 patients the drainage continued at a significant enough level to require further intervention. One patient demonstrated a clear decrease in overall drainage, but no change in peak drainage. $21 \%$ (4/19) showed no change in, or increased, effusion drainage rate while on octreotide. $16 \%$ $(3 / 19)$ were treated with intermittent subcutaneous injections, the remainder were treated with continuous infusion. No patients treated with subcutaneous injections demonstrated resolution. Two patients exhibited side effects temporally correlated to the initiation of octreotide - one patient with transient hypoglycemia, and one patient with diarrhea requiring decreased octreotide dosing. $37 \%(7 / 19)$ continued to have persistent drainage and subsequently progressed to surgical intervention (5) and/or death (2). Ten of the 19 patients had single ventricle physiology, 6 of whom responded to octreotide therapy. Conclusion: In children with chylous effusions after cardiothoracic surgery, treatment of the chylothorax with octreotide, in combination with dietary modification, resulted in a reduction of peak effusion drainage and eventual resolution in the majority of cases. The side effects observed were few and transient. This study would suggest that a future prospective trial be undertaken in order to determine the optimal dosing and timing of octreotide therapy. Until then, octreotide should be considered in treatment algorithms for chylothorax following cardiothoracic surgery.

\section{Children and Adolescents with Repaired Tetralogy of Fallot Report Health- Related Quality of Life Similar to Healthy Peers}

Elena Kwon, Cheryl Brosig, Mahua Dasgupta, Pippa Simpson, Kathy Mussatto, Margaret Samyn

Children's Hospital of Wisconsin, Medical College of Wisconsin

Background: Most patients with repaired Tetralogy of Fallot (TOF) have pulmonary regurgitation (PR) 
and right ventricular (RV) dilatation, but often lack symptoms. This study aimed to evaluate and compare parent-reported and self-reported quality of life (QOL) for pediatric patients with repaired TOF and determine the relationship with disease severity. Methods: Pediatric Quality of Life Inventory (PedsQL) Generic Core Scales were administered to 21 children with repaired TOF and their parents. Recent clinical data from cardiac magnetic resonance imaging (CMR), exercise testing, and B-Natriuretic Peptide (BNP) were reviewed. PedsQL scores were compared with normative data for healthy and chronically ill pediatric subjects. Results: Twenty-one parent-child pairs (8 males, 13 females) were assessed at a mean age of 11.4 yrs (8-18 yrs). Most could be considered healthy (76\% New York Heart Association class 1, with normal BNP $(25.8 \pm 30.1 \mathrm{pg} / \mathrm{ml})$, reasonable exercise tolerance, and preserved RV ejection fraction $(57.0 \pm 8.5 \%)$, despite moderate PR (35.6 $\pm 13.2 \%)$ and RV dilation $(114.4 \pm 26.8 \mathrm{ml} / \mathrm{m} 2)$ ). Self-reported QOL was similar to healthy subjects and, even better for school function. Compared to those with chronic illness, children with TOF overall reported better QOL, although not statistically significant for emotional function. Parental assessment of QOL for children with TOF was significantly lower than parents of healthy children in all categories except Emotional. Perceptions of TOF parents did not significantly differ from parents of children with chronic illness (Table 1). Social and School scores were significantly lower for TOF parents than for child self-report $(\mathrm{p}=0.015$ and 0.010 , respectively.) Age at initial right ventricular outflow tract surgery, number of operations, and prior shunt palliation negatively correlated with the parents' perceptions of school performance $(p=0.008,0.04$, and 0.007 , respectively). Parental scores for overall QOL, Physical, Emotional, and Social scores positively correlated with their child's VO2 max peak $\%$.

Table 1

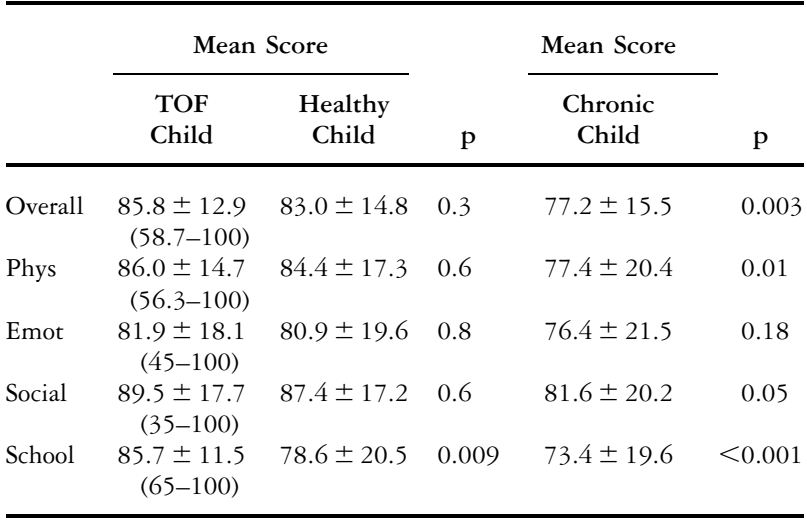

\begin{tabular}{|c|c|c|c|c|c|}
\hline & \multicolumn{2}{|c|}{ Mean Score } & \multirow[b]{2}{*}{$\mathrm{p}$} & \multirow{2}{*}{$\begin{array}{c}\text { Mean Score } \\
\begin{array}{c}\text { Chronic } \\
\text { Parent }\end{array}\end{array}$} & \multirow[b]{2}{*}{$\mathrm{p}$} \\
\hline & TOF Parent & $\begin{array}{l}\text { Healthy } \\
\text { Parent }\end{array}$ & & & \\
\hline Overall & $\begin{array}{r}78.1 \pm 14.9 \\
(42.4-100)\end{array}$ & $87.6 \pm 12.3$ & 0.004 & $74.2 \pm 18.4$ & 0.24 \\
\hline Phys & $\begin{array}{c}78.8 \pm 19.2 \\
(25-100)\end{array}$ & $89.3 \pm 16.4$ & 0.01 & $73.3 \pm 27.0$ & 0.2 \\
\hline Emot & $\begin{array}{c}76.2 \pm 17.2 \\
(45-100)\end{array}$ & $82.6 \pm 17.5$ & 0.09 & $73.1 \pm 23.3$ & 0.42 \\
\hline Social & $\begin{array}{c}73.6 \pm 22 \\
(30-100)\end{array}$ & $91.6 \pm 14.2$ & $<0.001$ & $79.8 \pm 21.9$ & 0.2 \\
\hline School & $\begin{array}{c}74.3 \pm 17.8 \\
(45-100)\end{array}$ & $85.5 \pm 17.6$ & 0.005 & $71.1 \pm 24.0$ & 0.42 \\
\hline
\end{tabular}

Conclusion: Clinically well children with repaired TOF consistently reported QOL similar to healthy children, while parental scores were lower. Thus, to ensure an active lifestyle, improve health perception, and prevent later acquired heart disease, comprehensive follow up should include psychosocial evaluation with cardiac rehabilitation.

\section{Nursing Interventions for Implementing the Berlin Heart in the Pediatric Intensive Care Unit}

Joshua Campbell, Michael Maymi, Melissa Reynolds

\section{Shands Children's Hospital}

Background: The Berlin Heart EXCOR ventricular assist device was approved by the Food and Drug Administration for compassionate use in children in the United States in 2000. In 2005, the FDA authorized a 15 center clinical research trial to determine the safety and effectiveness of the device in children. Currently, there are 52 centers in the United States who have used the device for compassionate use. Shands Children's Hospital at the University of Florida was not one of the designated 15 sites. However, the hospital was the first center in Florida to receive compassionate use approval in 2006 to place the Berlin Heart in a child with end stage cardiac failure as a bridge to transplantation. In the ensuing two years, three more children required placement of the device for severe circulatory failure.

Introduction of this new technology required an immediate, organized plan to address the educational needs of the Pediatric Intensive Care Unit (PICU) nursing staff through "just in time" training. Determining the priorities for care and understanding the effect of this therapy on these patients and their families proved to be difficult because of the paucity of literature regarding nursing management. Methods: The educational preparation for nurses and unique nursing care interventions for four children in the PICU who 
required placement of the device as a bridge to cardiac transplantation will be described. Nutritional requirements, protocol for laboratory specimens to guide therapy, cannula site dressing procedure, and the impact on parental caregiver stress and family dynamics will be explored. Four patients were admitted to the PICU with end stage cardiac failure from September, 2006, to the present ranging in age from 6 months to 9 years who required placement of the Berlin Heart EXCOR system. Medical records were reviewed to identify specific care needs of these children with particular attention to nutritional requirements, family dynamics, dressing to cannula sites, and laboratory values. The steps taken to educate the nursing staff to care for these patients were examined to determine their effectiveness for inclusion in an organized educational bundle for use in the future. Results: We found that nursing education for the entire PICU nursing staff initially focused on management of the ventricular assist device. Education evolved to include a holistic approach to the nursing care of these children and their families. Unique nursing interventions were required to address nutritional requirements, protocol for laboratory specimens to guide therapy, cannula site dressing procedure, and the impact on parental caregiver stress and family dynamics were identified. Conclusion: An organized, "just in time" educational bundle is required to adequately prepare nursing staff to care for children who require a ventricular assist device. To effectively meet the needs of the child and family, a multidisciplinary approach is essential. Attention to the nursing interventions required for care of cannula site dressings, nutritional needs, anticoagulation, and understanding of thromboelastogram (TEG) values will greatly improve patient outcomes.

\section{Let's Talk: Promoting Effective Communication in the Cardiac Operating Room Through Simulation}

Patricia Galvin, Emile Bacha, Robert LaPierre

\section{Children's Hospital Boston}

Background: Non-technical skills necessary for effective team work include leadership, communication, decision making and vigilance. Today's complex clinical environment requires enhanced communication skills to support teamwork and optimize outcomes. The operating room (OR) setting and cultural hierarchy present particular challenges to effective communication. Results of observational surveys in the Cardiac OR demonstrated below hospital benchmarks for effective communication skills. Recent publications highlight the need to improve communication through team training and use of simulation. Methods: Thirty-five articles relating to simulation and team training were reviewed and analyzed. Incorporation of simulation training was proposed to co-leaders of the Cardiac OR. Evaluation of simulation options pointed to high fidelity simulation being the most effective method to replicate the complex cardiac OR setting. A one day pilot was implemented to evaluate the benefits of simulation team training.

A pre-survey to identify challenging issues faced in the clinical setting was given to nursing and perfusion staff. A presentation including the presurvey results, a description of the importance of communication in patient safety, an introduction to the concept of team training to improve communication, an overview of simulation technology and the proposal for a pilot program using multidisciplinary simulation was put together. The use of Center for Medical Simulation (CMS) facility (Cambridge, MA) cost $\$ 6000$ charge per day. The proposal was accepted and financial support provided.

On the pilot day, nursing, anesthesia, perfusion and cardiac surgery were participants. CMS staff took the lead on the scenarios and discussions based on our communication goals. The team included two surgeons, one anesthesiologist, three nurses and two perfusionists. Three clinical scenarios were conducted. Debriefings led by a third party, provided a comfort level that allowed the team to develop trust with the experience and with each other. Results: Each scenario included a debriefing using guided discussion. Communication elements discussed included situational awareness, preoccupation, closed loop communication, use of names and leadership in the scenario. All eight participants completed a written Likert scale rating evaluation tool. The program was rated "excellent" by $100 \%$ of participants. The course goals and realism of scenarios was rated excellent by $87 \%$. The preview lecture and quality of debriefings were rated excellent by $100 \%$. The reflective discussions during the debriefings were considered a valuable part of the experience. Comments made at final debriefing and guided reflective discussion confirmed the value of training the entire team in simulation to improve communication with a focus on pediatric cardiac surgical issues. Conclusion: Effective communication is the key to effective teamwork. Promoting effective communication with the support of simulation is critical to maintaining patient safety. Training is supported by ongoing practice in simulation. The simulation was enhanced by comparing simulation experience to real clinical events. The debriefings were described as excellent opportunities. Engaging leaders in safety initiatives supports safe, reliable care and team building. Simulation training of Cardiac OR team members was approved and funded for 2009. 
Every member of the Cardiac OR team will participate in at least one simulation session. Observation measurements will determine outcome.

\section{An Early Warning Scoring System for Identification of Pediatric Cardiac Inpatients at Risk of Arrest}

\author{
Mary McLellan, J.C. Romano, R.E. Breitbart, \\ S. Reidy
}

\section{Children's Hospital Boston}

Background: Evidence indicates a majority of patients have signs of clinical deterioration 1-6 hours prior to arrest events. In 2007, Children's Hospital Boston participated in the Child Health Corporation of America Collaborative "Eliminating Codes and Associated Mortality on Inpatient Units" and developed the Children's Hospital Early Warning System (CHEWS). This scoring system combines objective and subjective assessment data that enhance the ability to detect clinical deterioration and potentially prevent it through the application of an escalation-of-care algorithm. In a collaboration between the Cardiovascular Program and the Program for Patient Safety \& Quality at Children's Hospital Boston, we assessed the implementation and efficacy of CHEWS in the pediatric cardiac population. Methods: Assessment of CHEWS and subsequent revisions occurred as 24 hour validation events. During validation, CHEWS scores were obtained by nursing every four hours on inpatient cardiology patients. Separately, the charge nurses, cardiac surgical nurse practitioners, cardiology fellows, and staff nurses identified patients they considered most acute and/or concerning. The patients' clinical courses during validation were also recorded. The clinicians' lists and patient clinical courses were compared to each other and to the CHEWS scores. Physicians and nurses modified the CHEWS tool to maximize the correspondence between the scores and the assessments of experienced clinicians. Results: Validation events demonstrated close correlation across clinical staff and patients' clinical courses. The initial validation event identified significant discrepancies between CHEWS scores and clinical data. Several of the patients identified on the clinicians' lists required an escalation of care during this 24 period; however, the CHEWS scores did not change. Analysis determined that CHEWS was not sensitive enough for the evolving changes of the cardiac population; specifically: 1) arrhythmias, a major risk factor for critical events, were not included; 2) oxygen supplementation scoring was not appropriate for infants, which represented $50 \%$ of the cardiac inpatients; and 3) the presence of cyanosis was not included. The tool was modified to the Cardiac CHEWS (C-CHEWS) to improve the capture of key data peculiar to this patient population. The second validation event, using C-CHEWS, had fewer discrepancies between clinical data and C-CHEWS scores. Analysis identified the presence of baseline versus acute onset cyanosis and/or arrhythmias were the variances between clinicians' concerns and CHEWS scores. An additional C-CHEWS revision encompassed changes in patients' baseline rhythms, oxygen saturation and oxygen supplementation. The last validation event had close correlation between clinical data and C-CHEWS score. Conclusion: A pediatric early warning system tool was adapted to pediatric cardiology patients through tool validation with clinician judgment and patients' clinical courses.

C-CHEWS helps identify early warning signs prior to deterioration in some pediatric cardiac patients. The tool does not replace clinical judgment, rather provides clinicians with an additional set of data to consider while caring for cardiac patients. Escalation of care based on early warning signs of deterioration enables faster proactive management of pediatric cardiology patients.

\section{Influence of Dedicated Pediatric Cardiac Intensive Care Unit on Patients' Outcome}

Maher Eldadah, Sheryl Leo, Kristina Kovach, Ricardo Argueta, Harun Fakioglu, William DeCampli

\section{Arnold Palmer Hospital for Children}

Background: The impact of a designated ICU for postoperative cardiac care in children is not clear. Our hospital (Arnold Palmer Hospital for Children APH) started a new Pediatric Cardiac Surgery program 5 years ago (September 2004). During the first 2 years postoperative care was accomplished within the confines of the general PICU (c-ICU). Subsequently, (starting 9/2006), a dedicated Cardiac ICU (d-ICU) was established. We looked at our experience during these 2 periods to see if the designation improved the outcome. Methods: We reviewed all medical records for postoperative cardiac admissions to the ICU during the first 4 years of the program (9/2004-9/2008). Data collected were: age, gender, diagnosis, type of cardiac surgery, RACHS, ventilator use, length of hospital stay, infections (wound, lines or ventilator related), need of cardiopulmonary support, returns to the operating room, emergent re-exploration of 
chest, delayed sternal closure, accidental extubations, re-intubation, and mortality. Statistical analysis was done using Pearson Chi square method. Results: There were 199 cases in the first 2 years and 244 in the following 2 years. We saw an increase in the number and complexity of cases during the second period (Table 1). However, incidence of morbidity declined as evidenced by the decrease in wound infection and need for chest re-exploration. In addition, mortality declined from $7 / 199$ (3.5\%) to $2 / 244(0.8 \%)$. $\quad \mathrm{p}<0.04$ and less children required $\mathrm{CPR}$ in the unit $(\mathrm{p}<0.01)$.

Table 1

\begin{tabular}{lrrl}
\hline Variable & c ICU & d ICU & P \\
\hline Age (mean D) & 839 & 764 & 0.44 \\
RACHS 4-6 & 28 & 47 & 0.08 \\
\#Ventilated Pts. & 113 & 149 & 0.32 \\
\#Extubation in OR & 69 & 95 & 0.8 \\
LOS (mean) & 19.4 & 26 & 0.26 \\
\#Line infections & 17 & 18 & 0.65 \\
\#VAP & 17 & 28 & 0.31 \\
\#Wound infection & 15 & 2 & 0.0003 \\
\#CPS & 8 & 4 & 0.13 \\
\#Re-exploration & 19 & 5 & 0.001 \\
\#Open sternum & 26 & 30 & 0.81 \\
\#Accidental extub. & 2 & 4 & 0.57 \\
\#Re-intubation & 28 & 27 & 0.34 \\
\#Death & 7 & 2 & 0.04 \\
\#Needed CPR & 12 & 4 & 0.01 \\
\hline
\end{tabular}

Conclusion: The designation of a specific area for postoperative cardiac care was instrumental in the growth and development of our cardiac program. This rapid change accomplished several crucial elements that lead to accelerated improvement in patient care thus a decline in morbidity and mortality. The program rapidly attained the critical manpower it needed to provide top quality medical and nursing care on a 24 hours basis.

\section{Evolving Approaches in the Management of Hypoplastic Left Heart Syndrome: Early and Intermediate Term Outcomes}

Evonne Krushansky, Diego Moguillansky, Ricardo Munoz, Victor Morell, Jacqueline Kreutzer

\section{Children's Hospital of Pittsburgh}

Background: Surgical palliation for hypoplastic left heart syndrome (HLHS) has evolved from Norwood procedure with BT shunt (NB), to Sano modification (NS) and hybrid procedure. Long-term outcomes and specific indications of each are not well known. We explored our results after NB and NS for HLHS and assessed risk factors for poor outcome. Methods: A retrospective review was performed on 45 consecutive patients who underwent surgical palliation for HLHS between July 2004 and March 2009. Data collection included surgical strategy, initial echocardiogram (right ventricular function, tricuspid regurgitation, aortic diameter, and atrial level shunt), birth weight, gestational age and survival. Kaplan-Meier curves were constructed for transplant free survival. A Cox model was used to evaluate predictors of survival. Results: NS $(\mathrm{n}=15)$ and NB $(\mathrm{n}=31)$ were performed with $11 \% 30$ day surgical mortality (all in NB group). There were 3 interstage deaths after NB and 1 after NS. Following Glenn procedure, there was 1 death in NB group, and 2 deaths and 2 transplants in the NS group. There was no mortality at Fontan. Although NS initial survival was better, there was less ongoing attrition after NB. The difference was however not statistically significant $(\mathrm{p}=0.68)$. The only predictor of survival in the Cox model was birth weight (hazard ratio 0.23 , $\mathrm{p}=0.004)$. Conclusion: Even though surgical mortality is lower for NS, the outcome is not different at intermediate follow up, and there appears to be a trend for ongoing steeper attrition for the NS group. Birth weight is the strongest predictor of outcome. Although complex surgical procedures can technically be performed on small neonates, low weight appears to still play a major impact on outcome.

\section{Indication for Advanced Cardiopulmonary Support in Infants and Children Determines Post-Support Survival}

Maher Eldadah, Monica Olson, Harun Fakioglu, William M. DeCampli

\section{Arnold Palmer Hospital for Children}

Background: Mechanical cardiopulmonary support (CPS) is utilized for hemodynamic collapse refractory to conventional medical management. In infants and children, CPS may be instituted before ("electively") or after ("emergently") loss of adequate spontaneous vital signs.

Hypothesis: Survival following CPS is higher when initiated "electively" than when performed "emergently". Methods: We reviewed the records of infants and children requiring CPS in our program from $6 / 2005$ to $6 / 2009$. Data collected included demographics, diagnosis, timing/place of initiating CPS, 30-day or in-hospital mortality, indications, complications and duration of CPS. Elective causes included failure to wean from cardiopulmonary bypass after operation or steady deterioration of cardiac function. Emergent causes included sudden 
cardiac arrest or sudden unrelenting hypoxemia, usually requiring closed chest compressions. We used chi-square test to compare groups. Results: 21 patients required CPS. Eight children were placed on CPS in the OR, 9 in the ICU, 3 in the catheterization lab and one in the emergency department.

\begin{tabular}{lclc}
\hline & \#Survived (\%) & \#Expired (\%) & Total \\
\hline Elective & $11(73.3)$ & $4(26.7)$ & 15 \\
Emergent & $1(16.7)$ & $5(83.3)$ & 6 \\
Mean CPS & 3.3 & 5.3 & \\
\hline
\end{tabular}

chi square $=5.6, p=0.018$

The location of CPS initiation and duration of CPS were not significant factors determining mortality. Conclusion: Outcome of CPS support in children depends strongly on whether CPS is instituted before or after loss of adequate spontaneous vital signs. With deteriorating heomdynamics, every effort should be made to institute CPS before loss of vital signs.

\section{Improved Nutritional Management of Infants with Congenital Heart Defects.}

Megan Donley, Jeff Anderson, Robert Beekman, Catherine Krawczeski

\section{Cincinnati Children's Hospital Medical Center}

Background: Failure to thrive is common in infants with congenital heart disease (CHD) and is especially prevalent among infants with a large ventricular septal defect (VSD) or complete atrioventricular canal defect (AVC). We recently began routinely involving a nutritional specialist at each clinic visit for infants with VSD or AVC. The purpose of this study was to assess the association between involvement of nutrition specialists as a part of the outpatient team caring for children with congenital heart disease and their pre-surgical weight gain. Methods: We performed a retrospective case series of all patients who underwent surgical repair of a VSD or AVC at Cincinnati Children's Hospital Medical Center (CCHMC) between 1/08 and 10/09 and who received their preoperative care at the Heart Institute at CCHMC. Implementation of routine outpatient nutrition consultation began in $9 / 08$. Infants who were premature ( $<36$ weeks gestational age) were excluded from the study. Weight and weight-for-age $\mathrm{z}$-score (WAZ) were recorded at birth, at each clinic visit and at the time of surgery. Comprehensive nutrition information including concentration of formula and route of delivery were recorded at each outpatient visit and at the time of surgery. Outcome variables included
WAZ at surgery, average daily weight gain between initial clinic visit and presentation for surgical repair and total post-operative hospital length of stay. Results: Thirty-five patients underwent surgical repair of a VSD or AVC during the study period; thirty-four patients met inclusion criteria. Patients cared for after implementation of routine nutrition consultation $(n=17)$ were similar to those cared for prior to this change $(n=17)$ in demographics $(53 \%$ female vs. $53 \%$ female), diagnosis of Trisomy 21 (23\% vs. 23\%), age at surgery $(5.2$ months vs. 5.0 months, $\mathrm{p}=0.9)$ and cardiac diagnosis (78\% VSD vs. 65\% VSD, $\mathrm{p}=0.13$ ). Patients who had routine involvement of a nutrition specialist prior to surgical repair had better pre-operative average daily weight gain, $17.4 \mathrm{~g} /$ day vs. $11.9 \mathrm{~g} /$ day $(\mathrm{p}=0.002)$ and shorter median hospital length of stay following surgical repair, 4 days vs. 7 days $(\mathrm{p}=0.02)$. Conclusion: Multidisciplinary involvement of a nutritional specialist routinely in the outpatient management of infants with a large ventricular septal defect or complete atrioventricular canal defect is associated with better preoperative weight gain and shorter hospital length of stay after surgery.

\section{The Impact of Fetal Diagnosis of Congenital Heart Disease on Parental Anxiety, Knowledge and Attachment}

Michael Willers, Bevin Weeks, Kathy Samuels, Linda Mayes, Lynette Tay

\section{Baystate Children's Hospital, Yale School of Medicine} and Yale Child Study Center

Background: Fetal diagnosis of congenital heart disease $(\mathrm{CHD})$ requires a significant investment of resources. Many reasons have been postulated to justify this use of resources, including the assumption that a parent whose child is diagnosed with CHD fetally, rather than postnatally, will be better prepared, resulting in improved parental well-being and enhancement of parent-infant attachment. This study tests the hypothesis that fetal diagnosis of CHD positively affects knowledge of the lesion, anxiety and parent-child attachment. Methods: Two groups of parents whose children who were 1 yearold and had CHD were enrolled. The first group ("fetal diagnosis," or FD) comprised 20 parents whose children were diagnosed fetally. The second group comprised 18 parents whose children were diagnosed postnatally ("postnatal diagnosis," or PND). Three instruments were administered: a modified Leuven Congenital Heart Questionnaire (MLCHQ) to assess parental knowledge; the Parenting Stress Index/Short Form (PSI/SF) to assess stress and 
attachment; and the Spielberger State-Trait Anxiety Inventory Form $\mathrm{X}$ (STAI) to assess anxiety. Results: There were no significant differences in MLCHQ scores between the FD and PND groups. However, significantly higher subscores on the MLCHQ were attained by those parents who were at home $(85 \%+$ / $-15 \%)$ and by those parents who worked in healthcare $(97 \%+1-8 \%)$, versus those who worked outside of healthcare $(66 \%+/-31 \%, \mathrm{p}=0.039$ and 0.01$)$.

There were no significant differences in PSI/SF scores between the FD and PND groups. However, parents who had completed college or graduate were less stressed than parents who had completed high school only $(11 \%$ vs. $0 \%$ vs. $43 \%, p=0.028)$. Those parents whose child was aneuploidic had significantly higher scores on the Parental Distress subscale $(43 \%$ vs. $6 \%, \mathrm{p}=0.035)$. Additionally, parents who scored lower on the MLCHQ scored higher on the Parental Distress subscale ( $40 \%$ vs. $4 \%, \mathrm{p}=0.014)$.

There were no significant differences in STAI scores between the FD and PND groups. However, there was an inverse association between educational level and current anxiety $(36.6+/-9.3$ for high school completion vs. $29.7+/-6.7$ for college completion, $\mathrm{p}=0.041$ ). Parents of aneuploidic children were more anxious in general $(48.8+/-2$ vs. $46.5+/-2.9$, $\mathrm{p}=0.44$ by 2-tailed t-test), and there was a trend toward higher current anxiety levels $(36.9+/-7.6$ vs. $31.2+/-7.8, \mathrm{p}=0.095)$. Those parents who scored higher on the MLCHQ when describing the heart lesion had lower current anxiety levels $(36.7+/-10.1$ vs. $30.1+/-6.2, \mathrm{p}=0.021)$. Conclusion: Fetal diagnosis of CHD does not affect parental knowledge of the lesion, parental anxiety, or parent-child attachment. However, certain demographic factors do impact these variables and can be useful when counseling families. Being a homemaker or employed in healthcare is associated with an increased understanding of a child's CHD. Being more knowledgeable about a child's CHD was associated with less parental stress. Parents of aneuploidic children were more likely to be anxious and stressed, as were those parents who had not completed college.

\section{How a High Risk Clinic in Addition to Home Surveillance Monitoring, Potentiates Care of Single Ventricle Patients}

Nancy Dobrolet, Jo Ann Nieves, Elizabeth Welch, Danyal Khan, Anthony Rossi, Redmond Burke, Evan Zahn

Congenital Heart Institute at Miami Children's Hosp and Arnold Palmer Hosp for Children
Background: Home surveillance monitoring (HSM) has been shown to identify pts at risk for inter-stage death following stage I palliation for hypoplastic left heart syndrome (HLHS); thereby, allowing for further evaluation and intervention. We developed a high risk clinic (HRC), combined with HSM, for all pts following any type of staged, neonatal heart operation or catheterization procedure. Methods: From January 2006 to the present, pts following a staged operation/catheterization (Norwood, shunt +/- Damus Kaye Stansel, hybrid Norwood or ductal stent), are discharged with scheduled follow up in our HRC, in addition to follow up with the referring cardiologist, until the time of their stage II or corrective operation. This clinic is in combination with our HSM of pulse oximetry and weight. Patients were compared to a historical cohort of pts who had undergone the same types of procedures between 01/2002-12/2005 (preHRC) but were followed solely by their referring cardiologist. Near miss events (NME) were defined as unplanned admissions which required an intervention, operation or cardiopulmonary support. Results: Interstage At Home Death

Inter-stage, at home deaths occurred in 2/44 (5\%) Norwood and 4/65 (6\%) shunt pts during the preHRC years. For those pts followed in our HRC, there were no Norwood inter-stage deaths $(\mathrm{n}=17)$ and only $1 / 34(3 \%)$ shunt pts who died at home.

Near Miss Events: During pre-HRC years, NME occurred in 2/44 (5\%) Norwood pts and 7/65 (11\%) shunt pts; compared to $7 / 17$ (41\%) Norwood pts $(\mathrm{p}<0.001)$ and $5 / 34(15 \%)$ shunt pts followed in HRC. Of 22 NME during the HRC years (including those in ductal stent and hybrid Norwood pts below), 7 (32\%) were direct admits from the HRC to our cardiac intensive care unit.

Inter-stage Catheter Intervention: Inter-stage catheter interventions were performed in the pre-HRC years in 8/44 (18\%) Norwood and 5/65 (8\%) shunt pts compared to $7 / 17(41 \%)$ and $4 / 34(12 \%)$ during the HRC years respectively.

Ductal stents and Hybrid Norwoods: No ductal stents or hybrid Norwood procedures were performed during the pre HRC years. Of the pts who had ductal stents $(\mathrm{n}=10)$ or a hybrid Norwood $(\mathrm{n}=4)$ followed in our HRC, $7 / 10(70 \%)$ and $3 / 4(75 \%)$ pts had a NME and $3 / 10(33 \%)$ and $4 / 4(100 \%)$ underwent an inter-stage catheter intervention respectively. All pts with ductal stents and hybrid Norwood procedures survived to the second stage of palliation. Conclusion: Initiation of a HRC, in combination with HSM, to a congenital heart center resulted in a statistically significant increase in the identification of near miss events in pts following Norwood operations, and in the identification of residual hemodynamic lesions amenable to therapy in other types of palliative procedures. 
Neonatal Correction of Tetralogy of Fallot in Slovakia - Early and Mid-term Results

Peter Skrak, M. Zahorec, L. Kovacikova

Pediatric Cardiac Center, National Institute of

Cardiovascular Diseases, Bratislava, Slovakia

Background: The optimal management of neonates with tetralogy of Fallot (TOF) who require surgical intervention remains debatable. The purpose of the study was to review the results of our institutional protocol to offer corrective surgery for patients suitable for complete repair. Methods: Retrospective analysis included neonates with variants of TOF: TOF with pulmonary stenosis (PS), TOF with pulmonary atresia (PA) and TOF with aplastic pulmonary valve (APV) who underwent surgery during period 1997-2008. Data are provided as median (range). Results: During the study period 34 neonates underwent surgical intervention: 1) corrective surgery 29 patients with weight of $3.5 \mathrm{~kg}$ $(2.7-3.8)$ at age 4 days $(2-27)$ at the time of surgery, and 2) modified Blalock-Taussig (MBT) shunt because of low weight $(\mathrm{n}=4$, weight $2.2-2.5 \mathrm{~kg}$ ) or diminutive pulmonary arteries $(\mathrm{n}=1)$. In all patients with corrective surgery the ventricular septal defect was closed completely and atrial septal defect partially. For right ventricular outflow tract various methods were used. Patients with TOF/PS $(\mathrm{n}=10)$ underwent transanular patch (TAP) placement $(n=9)$ or xenograft placement $(\mathrm{n}=1)$. Patients with TOF/PA $(\mathrm{n}=13)$ underwent TAP placement $(\mathrm{n}=8)$, right ventricle-pulmonary artery (RV-PA) direct anastomosis $(\mathrm{n}=4)$ or pulmonary homograft placement $(n=1)$. Patients with TOF/APV $(n=6)$ underwent anteposition of pulmonary arteries $(n=4)$, pulmonary $(n=1)$ or aortic $(n=1)$ homograft placement, TAP placement $(\mathrm{n}=2)$, RV-PA direct anastomosis $(\mathrm{n}=2)$, and aortopexy $(n=1)$. Patients were followed up for 6.3 years (0-12.3). Three patients (two with TOF/PS, one with TOF/APV) died in postoperative period. One patient (TOF/PA) died five months after surgery. Thirty-day survival of the whole group is $89.6 \%$, five-year survival is $86.2 \%$. During postoperative period patients required mechanical ventilation for 4 days (1-20) and inotropic support for 5 days (1-14). Delayed sternal closure was used in five patients. Length of stay in intensive care unit was 7 days (1-25) and hospital stay was 14 days (1-84). During follow-up 8 patients required 7 surgical and 6 interventional procedures. Freedom from reintervention is $80.7 \%$ at one year and $71.2 \%$ at five years after surgery for the whole group. One of five patients with MBT shunt died on the first postoperative day, three patients underwent later corrective surgery and are alive, and one patient was lost in follow-up. Conclusion: Neonatal correction of TOF can be accomplished with acceptable mortality, however further effort is needed to minimize later reinterventions.

\section{A Multidisciplinary Approach to Establishing a Clinical Practice Guideline for Endotracheal Tube Suctioning of the Pediatric Cardiac Patient}

Meena LaRonde, Annette Imprescia, Ravi Thiagarajan, Jeanne P. Ahern, Kevin Bullock

\section{Children's Hospital Boston}

Background: In response to a series of critical events following endotracheal tube (ETT) suctioning, a multidisciplinary task force was established within our pediatric cardiac intensive care unit (CICU) to evaluate the current practice of ETT suctioning in neonates and children with congenital and acquired heart disease. The task force consisted of CICU intensivists, cardiothoracic surgeons, nurse practitioners, clinical nurse specialists, nurse educators, respiratory therapists, and staff nurses. Methods: Over a period of six months an evidence based review of the literature was conducted with consultation from clinical experts in other like pediatric CICUs around the practice of ETT suctioning. Observations of ETT suctioning along with a survey exploring perceptions of best practice in the CICU were conducted. Key aspects of the suctioning process were then identified and examined. These included: identification of high-risk patients, frequency of "routine" suctioning, pre and post oxygenation, sedation, normal saline instillation, hemodynamic stability pre and post ETT suctioning, and documentation. Results: No evidence guiding the best practice of ETT suctioning was identified from the literature review and consultation with outside institutions. Wide variation in ETT suctioning was revealed through data from the conducted surveys. Guidelines were subsequently developed based on consensus of expert opinion within our task force. These included: clearly defining routine suctioning, pre and post oxygenation practices, criteria for normal saline instillation, measured depth suctioning, consistent use of end tidal $\mathrm{CO} 2$ monitoring, and individual sedation plans. In addition, patients meeting the following criteria were identified as vulnerable to adverse events occurring after ETT suctioning: pulmonary hypertension, shunted single ventricle physiology, $<$ 1year of age for the first 24 hours following cardiopulmonary bypass (CPB), risk for or actual left atrial (LA) hypertension, hypoxemia, hypercarbia, on inhaled 
Nitric Oxide (iNO), low cardiac output syndrome (LCOS), and on high frequency oscillating ventilation (HFOV). Conclusion: Establishing a clinical practice guideline has raised awareness of the high-risk nature of ETT suctioning in our patient population and has prompted daily discussions amongst the multidisciplinary care team. Ongoing assessment of these guidelines with possible modifications continues. Dissemination of this clinical practice guideline across disciplines remains a focus of ongoing education and communication.

\section{Beating the Bugs: Process Improvement to Decrease Catheter-Associated Bloodstream Infection in a Large Pediatric Cardiac Intensive Care Unit}

Torzone Andrea, Jodie Lantz, James Allard, Jean Storey, Jane Siegel, Michelle Macaluso, Jennifer Baker, Stephanie Dickson, Nadine Essary, Anna Jones, Laurie Schilling, Amy Smith

\section{Children's Medical Center Dallas}

Background: Catheter-associated blood stream infection (CA-BSI) is not only financially costly to an institution, but significantly contributes to patient morbidity and mortality during an intensive care unit (ICU) stay. Patients in the pediatric cardiac ICU are at heightened risk for CA-BSI due to complex diagnoses, young age, immunocompromised status, invasive procedures, and frequently accessed central lines. Rates of CA-BSI in our Cardiac ICU were above national average in 2006, 2007, and 2008. We hypothesized that the initiation of multiple interventions designed to heighten awareness and improve nursing and medical processes involving central line insertion and maintenance would decrease CA-BSI rates in the Cardiac ICU. Methods: Intensive effort was placed on decreasing CA-BSI rates in the Cardiac ICU. Current practices were evaluated and high risk areas identified. Our institution joined a NACHRI (National Association of Children's Hospitals and Related Institutions) Collaborative in June 2008 and incorporated CDCrecommended central line insertion and maintenance bundles into practice. Critical Care Services applied principles from the successful Lean Management Method including creating standard "prescriptions" for procedures such as obtaining blood cultures, tubing changes, CVL cap changes, and central line dressing changes, as well as encouraging process improvement by front-line staff. Additional process improvement initiatives included: auditing line insertion and maintenance; asking "Four Questions" about every patient's central lines every day at rounds; conducting multidisciplinary root-cause analysis reviews when a positive blood culture occurs; tracking "Days Since Infection" and informing staff daily via posters and email; initiating a "Scrub the Hub" campaign focusing on a vigourous 15 second alcohol hub-scrub-and-dry prior to every line access, and developing a front-line, unit-based nursing committee focused on spreading BSI initiatives. While all strategies have been effective in decreasing infections, the BSI Nursing Champions Team has had a major impact by dramatically increasing awareness, ownership, and education in the Cardiac ICU and providing leadership, candid feedback, and clinical solutions. Results: CA-BSI rates in the Cardiac ICU were 7.6 per 1000 line days in 2007 and 5.9 per 1000 line days in 2008 , respectively. To date, the 2009 rate is 3.0 per 1000 line days; Cardiac ICU has achieved a Unitrecord 89 consecutive days without infection. According to NACHRI data, estimated study benefits include: 12 prevented infections and over $\$ 406,000$ cost savings. The BSI Initiative is spreading to additional high-risk areas such as cath lab, interventional radiology, and OR through education and reinforcement of nursing and medical staff. Leadership and management fully support the BSI Initiative; staff ownership is the key to supporting excellent clinical practice. Conclusion: Implementation of multiple interventions has been successful in decreasing the incidence of CA-BSI in the cardiac ICU in a large tertiary care pediatric hospital. CA-BSI is a major quality indicator in the CICU. Multiple process improvement measures were implemented to decrease rates of infection. Effectively changing ICU culture is a challenge. Engaging nursing staff in the process has been an impressive strategy, in that the staff has taken ownership of the problem. Awareness and accountability are now high and CA-BSI rates are low.

\section{Ecologic Myocardial Protection: Minicardioplegia}

Brigida Aguerrevere, J. Russo, E. Medina, J. Iribarren, A. Sanchez, A. Alaña, J. Castejon

\section{Fundación De Todo Corazón Richard Gibson}

Background: Myocardial protection has always been a topic to discuss and a subject where changes in paradigms are not seen so often. It is an important issue of the surgical success that is reviewed and updated once in a while, maybe because sometimes we take for granted the nobility and tolerance to ischemia of the pediatric heart. Objectives: To share the experience of a team who believes that when more physiologic the heart is treated protecting it with minicardioplegia, faster and better the spontaneous activity recovery will be. Methods: From 
2008-2009 we evaluated 96 patients undergoing CPB. Mean complexity RACHS score: 2. Mean age was 3.7 years ( 3 month-12 years). The average time of cardiopulmonary bypass (CPB) was $33.7 \mathrm{~min}$ (14 min-78 min), mean xclamp time was $21.2 \mathrm{~min}$ (7 min-64 minutes). Mean nasopharyngeal and rectal temps were 34.5 y $35^{\circ} \mathrm{C}$ respectively. Pump prime consisted of Normosaline $0.9 \%$, Sodium bicarbonate, heparin and packed red blood cells to maintain a postdilutional hematocrit (Htc) of $30 \%$.

Isotermic Miniplegia was given antegrade every 20 minutes to all patients through a $1 / 4$ inch tubing line from the arterial port of the oxygenator membrane to a Roller head and to a manifold were we connected 3 lines:

1) Line pressure. 2) Table line with a bubble trap. 3) Line with a 20cc syringe, containing the arresting agents (Potassium $\mathrm{K}+$ and Magnesium), which were added to the blood cardioplegia circuit by means of an electrical syringe pump (ESP). Flow rate $(\mathrm{ml} / \mathrm{hr}$ ) of the ESP was calculated through a nomogram based on: Total blood volume to be delivered through the roller head $(20 \mathrm{ml} / \mathrm{kg}$ ), Time (minutes to deliver the blood volume) and target $\mathrm{K}+$ concentration $(20 \mathrm{meq} / \mathrm{L}$ for induction of arrest, $10 \mathrm{meq} / \mathrm{L}$ for its maintenance). Results: Spontaneous recovery rhythm was achieved in a mean of 23 seconds for 95 patients after cross clamp removal. Defibrillation was needed only for 1 case, no need of hemoconcentrator in all cases, final mean Hct was $31.74 \%$ and average Potassium blood level post minicardioplegia administration was $4.4 \mathrm{meq} / \mathrm{L}$. Conclusion: Myocardial protection strategy must balance the complexity of: effectiveness, simplicity, cost and evolution.

Administering blood rather than crystalloid solutions into the myocardium provides nutrients to the muscle. Aerobic blood cardioplegia shifts the oxyhemoglobin dissociation curve to the right. It has shown to be cost-effective because its functional simplicity at low cost and has no impact in the decision of the use of fluid removing devices like hemoconcentrators or cell saving devices. Custom-made cardioplegia circuits with a heat exchange integrated can also be used for this purpose.

Minicardioplegia resulted for us: An ecological way of treating the heart.

\section{Impact of Balloon Atrial Septostomy in Neonates with Transposition of Great Arteries}

Gurumurthy Hiremath, Girija Natarajan, Sanjeev Aggarwal

Children's Hospital of Michigan, Wayne State University School of Medicine
Background: Although balloon atrial septostomy (BAS) is commonly employed in neonates with transposition of great arteries (dTGA), there have been recent concerns regarding its efficacy and safety, especially since its presumed association with stroke in the peri-operative period. Prostaglandin E1 (PGE1) infusion is often used to alleviate hypoxemia in infants with dTGA. The introduction of pulse oximetry has resulted in a large increase in number of babies who receive PGE1 after septostomy and recent studies have suggested that PGE1 use has been associated with a longer postoperative hospital stay. Our objectives were a) to determine the immediate efficacy and impact on overall clinical outcomes of BAS in dTGA and b) to identify factors associated with successful BAS, defined as discontinuation of PGE1. Methods: This was a retrospective chart review of consecutive neonates with dTGA admitted to a tertiary care NICU between January 2000 and November 2008. Demographic, clinical, echocardiographic and outcome variables were compared between 3 groups: No BAS, Successful BAS and unsuccessful BAS using ANOVA, chi square and Kruskal Wallis test as appropriate. Results: Neonates $(\mathrm{n}=60)$ with dTGA were identified from the admission database; $42(70 \%)$ were males and the mean (SD) birth weight was $3271(603.2)$ grams. Of the $42(70 \%)$ infants who underwent BAS, $8(19 \%)$ were as "routine" and the remainder for desaturations on PGE1 ( $n=20)$ or restrictive mixing on echocardiogram $(n=14)$. PGE1 was successfully discontinued in $16(38.09 \%)$ infants following BAS at a median duration of 98 hrs. BAS resulted in a significant increase in the minimum $(64.2$ to $76.5 \%)$ and maximum (85 to $90 \%$ ) oxygen saturations and a drop in $\mathrm{FiO} 2$ (62 to $35 \%$ ) requirement and respiratory severity scores (RSS, calculated as mean airway pressure $\mathrm{X} \mathrm{FiO2}$ ) (4.6 to 2.1), 24 hours after the procedure (all $\mathrm{p}<0.05$ ). There was a significant increase in the shunt across PFO as anticipated $(\mathrm{p}<0.05)$. Three $(5 \%)$ infants died and $4(9.5 \%)$ developed strokes; all infants with strokes had undergone BAS. The duration of hospitalization, ventilation and oxygen requirements did not significantly differ between the three groups: a) no BAS, b) successful BAS and c) unsuccessful BAS. Neither did mortality ( 1 in each group) or stroke (1 in the successful BAS and 3 in the unsuccessful BAS group). A significant positive correlation was noted between the total duration of PGE1 and duration of hospitalization, post-operative ventilation and oxygen requirement as well as the maximum post-operative RSS (all $\mathrm{p}<0.03$ ). The only variables significantly associated with successful BAS were the presence of a ventricular septal 
defect (VSD) (50\% vs. $7.7 \%, \mathrm{p}=0.014)$ and male gender $(\mathrm{p}=0.021)$. Conclusion: Our data suggest that BAS is efficacious in relieving hypoxemia and reducing ventilatory support in infants with dTGA, but does not improve overall clinical outcomes. Presence of a ventricular septal defect and male gender are associated with successful discontinuation of PGE1 following BAS. Prolonged duration of PGE1 correlated with adverse post-operative outcomes. We speculate that limiting BAS for stringent clinical indications and aggressive weaning of PGE1 following BAS, especially in infants with a co-existing VSD would improve outcomes in this population.

\section{How Much Fluid do We Need for Immediate Post-operative Management in Tetralogy of Fallot?}

Jose Figueira, Ivan Machado, Iva Tolj, Maria Arape, Nakary Moreno

\section{Fundacion de Todo Corazon Richard Gibson, Hospital Especialidades Pediatricas}

Background: Cardiopulmonary Bypass (CPB) produces a systemic inflammatory response with consequences: endovascular lesion, alterated vascular permeability, with increase of fluid pass from intravascular to interstitial space. This effect increases the total body water volume in the interstitial space. Our aim is to show how in a homogenous group of patients, under CPB and Aortic XClamp (AXC) stress, by monitorizing simple hemodynamic parameters, we establish a fluid-management strategy that leads to a brief ICU stay and avoid inotropes. Methods: From 231 patients submitted to cardiac surgery, we retrospectively selected from January 2007 to August 2009, 34 cases $(14.72 \%)$ diagnosed with Tetralogy of Fallot (TOF). In order to assess intravascular space status and measure inflammatory response, we selected as variables: Blood Pressure (BP), Heart Rate (HR), Central Venous Pressure (CVP), Urinary Output (UO) and Urinary Density (UD). We related them to $\mathrm{CPB}$ and $\mathrm{AXC}$ times, blood glucose (BG) and white-blood count (WBC) to admission and discharge from our Intensive Care Unit (ICU). Results: All 34 cases underwent surgery on normothermia, with minicardioplegia. Infundibular patch (18 cases), transanular patch (10 cases), infundibular and pulmonary patch (6 cases). Management protocol includes: $2000 \mathrm{cc} / \mathrm{m}^{2} \mathrm{BSA}$ base hydration with saline $0.45 \%$ and dextrose $5 \%$, NSAID (Ketoprofen $2 \mathrm{mg} / \mathrm{Kg}$ ). Aim was to obtain normal UD, UO, HR, BP and CVP values. Our population (data expressed: median (min-max). Age: 2.1 (0.9-9) years; Weight: 11.5 (7.6-27.4) Kg; Height: 82.5 (63-132) cm; CPB time: 55.5 (38-109) minutes;
AXC time: 39 (20-80) min; Extubation time: 0 (0-24) hours; ICU stay: 21.5 (14-100) hours. UD (pre CPB): 1020 (1006-1034), UD post CPB: 1021 (1006-1035), UD on ICU admission: 1025 (1010 1036), 6 hours later 1030 (1015-1040). BG on ICU admission: $113(103-238) \mathrm{mg} / \mathrm{dl}$. BG on discharge: $110 \mathrm{mg} / \mathrm{dl}(76-197) \mathrm{mg} / \mathrm{dl}, \mathrm{p}=0.46$. HR: $118(93-$ 135) x'; Systolic BP: 97 (63-127) mmHg; Diastolic: 58 (48-94) $\mathrm{mmHg}$; CVP: $10(7-13) \mathrm{mmHg}$. Total fluids in 6 hours: $1422(776-2379) \mathrm{cc} / \mathrm{m}^{2} \mathrm{BSA}$; Theoretical total fluids calculated per patient: 257 (189-499) $\mathrm{cc} / \mathrm{m}^{2} \mathrm{BSA}$; UO in 6 hours: $2.1(0.4-6.6) \mathrm{cc}$ $\mathrm{kg} / \mathrm{h}$; WBC on ICU admission: 15350 (3600-32500)/ $\mathrm{mm} 3$; WBC on discharge: 12975 (3700-23800)/mm3 $(\mathrm{p}=0.117)$. There is a 5.72 times-increase in the total fluid administration during the first 6 postoperative hours compared to the theoretical-calculated values $($ median $=5.53, \mathrm{p}<0.05)$. One patient died $(1 / 34$; 2.94\%). Conclusion: On selected patients, $\mathrm{CPB}$ and AXC time are no important variables to consider regarding severity of the inflammatory response. It can be better observed through clinical outcome, WBC and BG. With normal renal function, UD is not relevant as an isolated parameter, but together with all the before mentioned variables, it permits to properly assess intravascular space. Adequate values of UD, UO, BP, HR and CVP, carried about a five-fold increase of total fluid administration. Early extubation, a friendly environment with parental presence and adequate analgesia, led to a satisfactory final result: short ICU stay and good clinical outcomes. The amount of fluids given as part of a simplified cardiac surgery postoperative protocol is safe for our patients and does not cause late complications. Reduced ICU stay was possible, obtaining final cost reduction, important aspect to be considered, especially in costconstrained environments.

\section{Hemodynamic Significance of Low Peak Doppler Flow Velocity in the Proximal Sano Conduit Following the Norwood Sano Surgery in Patients with Hypoplastic Left Heart Syndrome}

Shyam Sathanandam, Wei Cui, Andrew Van Bergen, Michel Ilbawi, David Roberson, Dhaval Patel, Alex Javois, Tarek Husayni

\section{The Heart Institute for Children, Oak Lawn, IL}

Background: The Sano modification of the Norwood operation has become a well established first step palliation for hypoplastic left heart syndrome (HLHS). It involves the use of a narrow Gore-Tex conduit $(4-6 \mathrm{~mm})$ from the right ventricle $(\mathrm{RV})$ to the pulmonary artery (PA). Hemodynamically, the 
highest resistance to pulmonary flow should be in the proximal Sano conduit producing high Doppler flow velocity at this point. The objective of this study is to determine the hemodynamic significance of low proximal Sano Doppler flow velocity and its clinical implications. Methods: We measured Doppler derived peak Sano gradients on 53 HLHS survivors following Norwood Sano surgery from April 2004 to April 2009. All these patients underwent cardiac catheterization within 2-4 weeks of the Doppler study before referral for the bidirectional Glenn surgery. We confirmed Doppler measured peak Sano gradients by cardiac catheterization. McGoon ratio using the narrowest PA branch diameter and Nakata index using PA diameter at the level of the hilum of the lungs were calculated in all patients from angiograms. Balloon angioplasty of the Sano and PA branches was performed when necessary. Results: The Sano conduit size ranged from 4-6 mm. Low Doppler peak Sano gradient $(</=30 \mathrm{mmHg})$ was found in 22 of the 53 patients (41.5\%). It correlated with smaller Right PA diameter $(3.2+/-1.2$ vs. $4.5+/$ $-1.8) \mathrm{p}=0.02$ and Left PA diameter $(3.4+/-1.2$ vs. $5.6+/-2.1) \mathrm{p}=0.007$. It also correlated with smaller McGoon ratio $(1.38+/-0.26$ vs. $1.66+/$ $-0.33) \mathrm{p}=0.006$. However, it did not correlate with the Nakata index $(\mathrm{p}=0.086)$. We confirmed Doppler derived Sano gradients by cardiac catheterization in 27 of the 53 patients. Ten of the 53 patients $(19 \%)$ needed either balloon angioplasty of the Sano or branch PA with good immediate results. Only 2 patients needed an additional shunt surgery before bi-directional Glenn. Conclusion: Low peak Doppler flow velocity in the proximal Sano correlates with the presence of either distal Sano stenosis or proximal branch PA stenosis. It is associated with smaller McGoon ratio but not Nakata index. This is possibly because these patients have well developed distal PA branches despite some proximal narrowing. Balloon angioplasty of Sano is associated with good immediate results.

\section{Beyond Borders: Nursing experience in a Cardiac Surgery Satellite Program}

Rita Castro, D. Monerrosa, M. Velásquez, C. von Scanzoni, J. Iribarren, J. Figueira, I. Machado, A. Páez

Fundación De Todo Corazón Richard Gibson, Hospital de Especialidades Pediátricas

Background: Worldwide, there is a lack of qualified nurse professionals, especially in subspecialties as pediatric cardiology and surgery. In 2006, our program: "Proyecto de Todo Corazón" (Caracas, Venezuela) developed a satellite program in the "Hospital de Especialidades Pediátricas", Maracaibo. By then, they just had a Cardiology Unit not able to perform Cardiac Surgery. We had a 25-member qualified multidisciplinary team with a six-year experience: 4.117 evaluated patients and 662 performed surgeries. Funding for the program was obtained from external private sources. Aim of this paper is to evaluate and share the challenge experienced for us as a team, especially for our nurse group, in the development and training of new nurse professionals in this different environment and the impact in the outcome of the program. Methods: Review the general data from February 2006 until May 2009 by assignments, working days and performed surgeries, patient's age and weight, extubation time, Intensive Care Unit (ICU) stay, mortality rate, nursing team activity schedule and questionnaires delivered to them about time in the program, job satisfaction and training quality. Results: Assignments 43; Working days: 91 (mean 2.6 patients); performed surgeries: 241 (on pump: 164 peripherals: 77) Age: 0-18.7 years (mean 4.7 years), weight: $3.2-68.5 \mathrm{Kg}$ (mean $16.3 \mathrm{Kg}$ ). Extubation time: 0-24 hours (mean 0.5 hours) ICU stay 1-100 hours (mean 16.3 hours) Mortality rate: $3 \%$. Trips (one hour flight time) were organized monthly, three to four working days. Patients were previously selected based on type of defect, surgical complexity, and clinical status. Over time we increased number of surgeries and their complexity. Nursing team members: 16. From Maracaibo, 3 candidates were chosen for the roles of scrub nurse, anesthesia and outpatient clinic and program management. One nurse of our team (clinical specialist) is in charge of the program coordination. Our nurses had the opportunity to adopt different roles through the continuum of care (cross-training between these three subspecialties). One nurse left for a one year perfusion training and returned to our perfusion group. Each nurse from the "Maracaibo Team" was paired with one of the "Caracas Team". An average of 6 nurses in the ICU were planned to fill in morning, evening and night shifts. Recently two new nurses from Maracaibo were enrolled. Clinical pathways are applied to analyze procedures and protocols as well as family centered care strategy, in order to warrant a safe and friendly environment for our children.

Reviewing the answered questionnaire, all considered the program as an excellent experience, the subspecialty training important, adequate and updated. Their motivation to belong to it, as a combination of professional development, social commitment and economical satisfaction. Conclusion: For optimal outcome and good surgical results, it is absolutely 
necessary to have appropriately qualified team work, but also be prepared to assume different roles (surgery, anesthesia and Intensive Care). It can be accomplished with pleasure and guarantees optimal continuity and quality care for the patient through his hospital stay. Nursing team considered that the program has also a positive impact in job retention and satisfaction.

\section{Feeding Challenges in Infants following the Norwood Operation}

Karen Uzark, Nancy Rudd, Marsha Elixson, Jennifer Strawn, Jo Ann Nieves, Cathy Smith, Sandra Staveski, Patricia O'Brien, Elizabeth Tong, Yu Wang, Richard Ittenbach

Cincinnati Children's Hospital Medical Center, Children's Hospital of Milwaukee, Egleston Children's Hospital, Children's Hospital and Medical Center, Omaha; Miami Children's Hospital, Arnold Palmer Children's Hospital, Lucile Packard Children's Hospital at Stanford, Children's Hospital Boston, University of California - San Francisco

Background: Feeding difficulties and inadequate nutrition in infants after the Stage I Norwood operation can adversely impact the outcomes of surgery. In a multicenter study, we examined feeding strategies and outcomes in infants following the Norwood operation. Methods: A cohort of 158 infants who survived to discharge after Stage I Norwood were enrolled. There were 4 interstage deaths and 3 exclusions between Stage I discharge and Stage II surgery. Data regarding infant weight and feeding strategies, postoperative feeding difficulties and complications, hospital length of stay, and survival to Stage II were collected from medical records and prospectively from parent logs following Stage I discharge. Results: Mean Stage I weight was $3.2 \pm 0.47 \mathrm{~kg}$, mean age $6.2 \pm 4.3$ days (d) with an RV-PA shunt in $86(57 \%)$ of infants. Mean weight at Stage I discharge was $3.46 \pm 0.56 \mathrm{~kg}$. Discharge feeding regimen was oral $(\mathrm{O})$ only in 79 including 4 breast feeding (52\%), nasogastric or gastrostomy tube (T) only in $22(15 \%)$, and oral + tube supplementation $(\mathrm{O}+\mathrm{T})$ in $51(33 \%)$. There were no significant differences between the feeding groups related to Stage I age, weight, or shunt type. Oral motor dysfunction (OMD) was reported in $17 \%$, swallowing dysfunction (SD) in $15 \%$, vocal cord paralysis (VCP) in $17 \%$, and GE reflux in $54 \%$. Discharge medications included a prokinetic agent in $34 \%$ and an $\mathrm{H} 2$ blocker in $58 \%$. Tube only feeders had a significantly higher incidence of OMD $(\mathrm{p}<0.0001)$, SD $(\mathrm{p}<0.0001)$, $\operatorname{VCP}(\mathrm{p}=0.003)$, and GE reflux $(\mathrm{p}<0.001)$ than $\mathrm{O}$ only infants and a significantly higher incidence of OMD $(\mathrm{p}<0.001)$, SD $(\mathrm{p}<0.001)$, and VCP $(\mathrm{p}<0.02)$ than $\mathrm{O}+\mathrm{T}$ feeders. $\mathrm{T}$ only feeders had a significantly longer Stage I median length of stay (36 d) in comparison to $\mathrm{O}$ only ( $22 \mathrm{~d}$ ) and $\mathrm{O}+\mathrm{T}$ infants $(24 \mathrm{~d}), \mathrm{p}<0.001$, but Stage I discharge weight did not significantly differ between the groups. Conclusion: Feeding difficulties are common following the Norwood I operation and may prolong hospital length of stay. GE reflux was noted in more than half of infants post Norwood. OMD, SD, VCP, and GE reflux are more common in infants discharged with tube feedings. Early systematic evaluation of these problems may facilitate appropriate tailoring of feeding regimens and may contribute to decreased hospital length of stay.

\section{Predictors of Exercise Induced Hypertension in Repaired and Native Coarctation of the Aorta}

Jon Wagner, Mark Gelatt, Richard Sabath, Kelli Teson

\section{Children's Mercy Hospital, Kansas City, Missouri}

Background: Coarctation of the Aorta (CoA) varies in terms of clinical severity and age at presentation. It is unclear whether certain factors may predict resting and exercise-induced hypertension later in childhood. The correlation between time of surgical repair, residual echocardiographic findings, resting systolic blood pressure and maximal exercise systolic blood pressure were assessed. Methods: A retrospective chart review of $26 \mathrm{CoA}$ patients (20M, $6 \mathrm{~F})$ who underwent exercise testing from 19972009 was performed. Clinical records, echocardiograms and exercise test results were included. In patients with multiple exercise studies, the most recent exercise test was used for analysis. Systolic hypertension at rest (HTN-R) and with exercise (HTN-Ex) were defined as $>95 \%$ predicted. Association between these factors was assessed by Chi Square calculations. Results: Comparison was made between 9 patients surgically repaired $<6$ months vs. 13 repaired $>6$ months (11 surgical; 2 angioplasty) and 3 unrepaired. 2/9 repaired $<6$ months had HTN-R vs. $6 / 16$ in those without early surgery $(\mathrm{P}=0.43) . \quad 5 / 9$ patients repaired $<6$ months had HTN-Ex vs. 10/16 in those without early surgery $(P=0.76) .6 / 20$ males and $2 / 6$ female patients had HTN-R $(\mathrm{p}=0.87)$. 12/20 males and $4 / 6$ females had HTN-Ex $(\mathrm{p}=0.76)$. Comparison was made between 16 patients with mild $(<30 \mathrm{mmHg})$ systolic gradient (MILD) in the descending aorta on echocardiogram vs. 10 patients 
with gradient $>30 \mathrm{mmHg}$ (MOD). $3 / 16$ MILD had HTN-R vs. 5/10 MOD (P=0.09). 8/16 MILD had HTN-Ex vs. 8/10 MOD $(\mathrm{P}=0.13) .12 / 18$ patients who were normotensive at rest had HTNEx vs. 4/8 HTN-R developed HTN-Ex $(\mathrm{P}=0.42)$. Conclusion: Although $31 \%$ of our patients had hypertension at rest and 62\% developed hypertension with exercise, no correlation was found between age at time of surgery, resting systolic blood pressure and HTN-Ex. Although it did not reach statistical significance, there is a suggestion that patients with $>30 \mathrm{mmHg}$ peak systolic gradient across the aortic arch are more likely to have both HTN-R and HTN-Ex. This study illustrates the importance of exercise testing in CoA patients.

\section{Cardiac Evaluation in Patients with Turner Syndrome by MRI}

Robert Bishop, Wasim Khan, Chandrakant Patel, John Lane, Philip Smith

\section{Children's Hospital Medical Center of Akron/ NEOUCOM}

Background: It has been known that patients with Turner syndrome have a variety of cardiac defects including coarctation of the aorta and bicuspid aortic valve. However, due to the limitation of transthorasic echocardiogram, especially in older children and young adults, there is a need to have an imaging modality that can overcome these limitations. We hypothesize that with its recent advancements, MRI can provide anatomical detail of the heart superior to transthorasic echocardiogram in patients with Turner syndrome. Methods: Eleven patients underwent cardiac MRI by using $1.5 \mathrm{~T}$ machine manufactured by Philips. Patients were age 10-22 who carried a diagnoses of Turner syndrome based on physical findings and karyotype. These 11 patients were referred from either their primary care pediatrician or from the Endocrine Clinic at Akron Children's Hospital between October 2008-August 2009. Each patient had a complete physical examination prior to the MRI scan. Patient's were interviewed by MRI technicians for the possibility of foreign metal objects, allergies to contrast material and renal abnormalities. Prior to the MRI, the need for conscious sedation was based on patient's age. It was planned not to sedate patients $>10$ years old with no cognitive defects.

A standard protocol was designed to evaluate cardiac defects: Non-ECG gaited 3-plane and interactive localizing SSFP sequence. EKG-gated, breath-hold SSFP cine: Ventricular two-chamber, four-chamber, short axis and candy cane were performed. Also, RVOT and LVOT were performed. Straight axial views were obtained. EKG-phase velocity flow measurement was performed in the aortic root and the main pulmonary artery was performed. 3-D gadolinium enhanced MRA of the chest was performed. Each study was analyzed and reviewed at a 3-D work station by a MRI trained cardiologist. Results: Eleven patients with Turner syndrome were evaluated by cardiac MRI. Three patients were found with previously undiagnosed PAPVR (27\%). Three new cases of mild coarctation of the aorta were diagnosed $(27 \%)$. Other cardiac lesions found during cardiac MRI and not seen on prior ECHOs include RV dilatation (18\%), RA dilatation (9\%), LVH (9\%) dilated aortic root $(18 \%)$, biscuspid aortic valve $(18 \%)$ and dilated proximal descending aortic arch (9\%). 1 had no cardiac defects (12.5\%). Conclusion: It has been reported that the prevalence of PAPVR in patients with Turner syndrome ranges from $13-25 \%$. Our study shows a slightly higher incidence of PAPVR $(27 \%)$ and there was also a $27 \%$ incidence of mild coarctation that was unknown prior to cardiac MRI. Our study proves that with the recent advancements in the field of cardiac MRI, it is a superior imaging modality in the evaluation of cardiac defect in patients with Turner syndrome.

\section{Use of Basic Stimulation Concept in Nursing Care in Pediatric Cardiac Intensive Care Unit}

Miroslava Hargasova, Kovacikova Lubica

Pediatric Cardiac Centre, National Heart Centre, Slovakia

Background: The Basic stimulation concept (BS) has been implemented into the care of seriously physically and intellectually handicapped children by German Professor Andreas Fruhlich, special educator teacher. The method is focused on support of sensory communication in those with perceptive difficulties. Later, the concept was adapted to nursing practice by a nurse, Christel Bienstien. The aim of our work is to report initial experience with the use of Basic stimulation concept in Pediatric cardiac intensive care unit. Methods: During a period of 3 years various methods of Basic stimulation were used in children after cardiac surgery. Results: "Initial touch" as a mean of tactile stimulation was used in every patient. Before any manipulation a child was touched (usually on the forehead, shoulder, or arm) and spoken to in an attempt to establish a fearless relationship. Patients requiring long-term analgesia and sedation due to cardiovascular and respiratory instability are prone 
to withdrawal symptoms. We adapted positioning techniques which tied in with prenatal experiences "nesting, or mummy". Soft pillows, blankets and toys were used for the positioning. Somatic stimulation such as whole body soothing massages were intended to calm the patient. Massage stimulating breathing was introduced to patients who were weaned from ventilator support. Contact breathing in combination with vibrating techniques during exhalation were used in children with atelectasis. Neurophysiological stimulation such as stimulating massages found its' place in patients after thromboembolic episodes and paresis. Neonates after reconstructive surgeries on aortic arch have significant risk of swallowing difficulties, and intolerance of feeding due to recurrent laryngeal nerve dysfunction. Oral stimulation as a mean of preservation of sucking reflex was introduced to them as well as to newborns fed via nasogastric tube immediately after their birth. Mothers were taught the techniques of oral stimulation as well. For olfactoric stimulation techniques we used the mother's scent as stimulus familiar to the children to calm them. Vestibular and vibrating stimulation was mainly used in long-term patients. It was performed by rocking baby's chair or a vibrating chair. The ideal option was placing the child into mothers' arms. Initially we applied the method only to spontaneously breathing patients without chest-drains, and then gradually to children on non-invasive ventilation. Nowadays, even ventilated children with invasive access are cuddled by their moms. In auditive stimulation we encouraged parents to read, sing and talk to the child. Healthcare professionals introduced relaxing music, calm voice, lullabies, sounds of nature to all children as tolerated. Visual stimulation was carried out by placing pictures, familiar objects or stimulating lights into patient's bed. Conclusion: Three years of experience has shown that methods of Basic stimulation are highly applicable in Pediatric cardiac intensive care unit. We think that Basic stimulation concept is about organizing usual care in a way when health care professionals are empathic and focused on psychosomatic needs of patients and their families.

\section{The Genesis of a Pediatric Cardiac Intensive Care Nurse Practitioner Program}

Lauren Westgate, Meena LaRonde, Kathryn Barrett, Joylyn Headings, Whitney McCormick, Kim Reiser, Kathy Whelan, Jeanne Ahern

\section{Children's Hospital Boston}

Background: Our institution has had long-standing success with nurse practitioners (NP), specifically within the cardiovascular program (CVP). Pediatric intensive care units have historically utilized nurse practitioners as integral members of both critical care medical and surgical teams. Due to the expansion of critical care beds, an increase in patient acuity, and in the setting of restrictions in resident work hours, NPs were integrated into our cardiac intensive care unit (CICU). Methods: A literature review was performed to examine the history of advance practice nursing in pediatric critical care. Personal interviews were conducted to gain a perspective on the early experience of nurse practitioners within the CVP. The role of the NP specific to the CICU was further defined using established scope of practice, documentation of practice privileges and a review of non-clinical responsibilities that include both unit-based and institution wide committee participation, continuing education and research contribution. Results: The NP program was piloted in 2006 by an experienced staff nurse who recently transitioned to a NP role. Once the NPs were integrated as permanent members of the cardiac critical care team, a scope of practice and job description were formally established.

Additional NP positions were created to support the ever-changing needs of the unit. Our current structure includes six NPs with a variety of educational and clinical backgrounds. NPs currently staff the unit up to eighteen hours a day, seven days a week. The expansion of the NP program has promoted continuity of patient care and has allowed for consistent involvement in unit-based and institutional wide committees and research. Most importantly, integrating NPs into the CICU has helped bridge the gap between nursing and medicine and has promoted collaboration within the multidisciplinary team. Conclusion: Since the inception of the CICU NP program, changes in structure and role have been made to meet the dynamic needs of the CICU. Future endeavors for this program include assessing and evaluating the effects of the program on patient care, collaboration and contributions to the CVP.

\section{Gastrostomy Tube Following Neonatal Cardiac Surgery: Incidence and Risk Factors}

Michael Walsh, Scott M. Bradley, Myla Ebeling, Thomas C. Hulsey, Andrew M. Atz

\section{Medical University of South Carolina}

Background: Feeding difficulty is a common source of morbidity in neonates undergoing cardiac surgery. Its implications are far-reaching - failure to thrive, increased length of hospital stay, the need 
for additional non-cardiac surgery, and even mortality. Gastrostomy tube (G-tube) placement is a common end-point for patients with aspiration, gastrointestinal tract malformations, and inability to achieve adequate caloric intake. We reviewed our institutional experience with neonatal cardiac surgery to delineate the incidence of and risk factors for G- tube placement in this population. Methods: The electronic medical records of all patients who underwent cardiac surgery in the neonatal period (first 30 days of life) from August 2004 to May 2007 were reviewed. A total of 141 patients were identified, after excluding patients with only the ligation of an arterial duct. The primary endpoint was placement of a G-tube during initial hospitalization. Multiple pieces of data were gathered for each patient, including patient data (age at surgery, birth weight, gestational age), surgical data (use and length of cardiopulmonary bypass, single ventricle vs. biventricular repair, need for extensive aortic arch reconstruction), and postoperative data (length of mechanical ventilation, ICU and hospital stay). Results of modified barium swallowing studies, fiberoptic laryngoscopy, and upper gastrointestinal series were also recorded. Results: Of 141 patients undergoing cardiac surgery in the neonatal period, $31(22.0 \%)$ received a G-tube before hospital discharge. G-tube placement was not statistically different between those with single ventricle procedures $(17 / 61,27.9 \%)$ and those with biventricular repairs (14/80, 17.5\%, $\mathrm{p}=0.14)$. Patients with a confirmed diagnosis of a genetic syndrome were 3.4 times more likely to receive a G-tube $(52.2 \%$ vs. $15.4 \%, \mathrm{p}<0.01)$. Of particular interest, five out of nine patients with deletion of chromosome 22q11 received a G-tube. Surgeries involving extensive reconstruction of the aortic arch were associated with an increased risk for G-tube $(30.9 \%$ vs. $16.3 \%, \mathrm{p}=0.04)$, as was a modified barium swallow test positive for aspiration $(52.9 \%$ vs. $17.7 \%, \mathrm{p}=0.01)$. Additionally, neonates with left vocal cord paralysis seen on fiberoptic laryngoscopy were 1.7 times more likely to require a gastrostomy than those without a positive test $(35.7 \%$ vs. $20.5 \%, \mathrm{p}=0.19)$. Duration of post-operative ventilation was longer in patients that eventually received a G-tube (median 4 days vs. 2.5 days, $\mathrm{p}=0.02$ ). Total hospital stay was also prolonged in the G-tube population (median 50 days vs. 20.5 days, $\mathrm{p}<0.01$ ). Age at the time of cardiac surgery, birth weight, and gestational age were not associated with need for G-tube tube placement. Conclusion: Gastrostomy tube placement is pursued for a number of reasons in the population of neonatal cardiac surgery patients. This data suggests that patients with a genetic syndrome, especially the 22q11 deletion, are significantly more likely to receive a G-tube. We also noted that the common triad of extensive aortic arch reconstruction, left vocal cord paralysis, and aspiration is strongly associated with G-tube placement. Given the prolonged hospital stay in this population, an early referral for $\mathrm{G}$-tube in these higher risk patients may be warranted to reduce total length of hospital stay and overall cost of hospitalization.

\section{Everyone on the Same Page: A Daily Plan of Care Sheet Revisited}

J.-S. Rim, Sharon Barton, Ashley Elford, Erin Forster, Marlene Mahle, Mary Stuart, Michelle Ulrich, Christina Walden

\section{The Cardiac Center, The Children's Hospital of Philadelphia}

Background: Nurses have a unique role in facilitating information sharing between patients, families and the interdisciplinary patient care team. With that in mind, our bedside nursing staff chose family/nurse communication as a top priority for investigation. Admission of a child to the hospital could be the most stressful time of a parent's life, and we believed improving communication and providing the family with a venue to have their questions answered in a timely manner could ease the burden. Additionally, lapses in communication have been associated with hospital errors. Clear communication between nurses and families can improve patient safety, increase adherence to the plan of care, decrease patient length of stay, and increase family satisfaction. Plan of care sheets have been devised for use in our stepdown and cardiac intensive care units but usage was noted to be $90 \%$ in our ICU and $10 \%$ in our step down unit. With bedside nurses having the unique opportunity of spending the most time with the patient and family, nursing looked to revise the plan of care sheet to address all documented elements of effective communication. Methods: Using the IOWA model of evidence-based practice, the research committee began the process of searching, reviewing, and grading existing evidence for best practices in family-nurse communication about the plan of care. Summary tools developed by Johns Hopkins served as our framework for summarizing and grading research and non-research evidence. With the findings from the literature review, our present plan of care sheets for our cardiac step-down and intensive care units were reviewed and modified. Results: Literature emphasized the importance of a daily goals sheet using a shared decision making 
model, the benefit of continuity of caregivers, and surmised that by asking parents for their questions and opinions while giving frequent updates on the patient's plan of care decreased parents' stress level.

More specifically, some of the aspects we included on our revised plan of care sheets include detailing who will update the family, asking team members to perform a "read back" of the plan to ensure accuracy, and having a spot for families to write their specific questions for the day on sheet to relay to the medical team. Conclusion: Upon final review by other Cardiac Center nursing stakeholders, our nursing research committee plans implementation of the sheet while measuring aspects of improvement. As we forge into the future more aware of ways to build positive relationships between the nurse and family, our ultimate goals remain to improve patient safety, foster healthy relationships, lessen parental stress, and affect patient outcomes positively.

\section{Morbidity and Mortality after Hospital Discharge for Neonatal Congenital Heart Surgery is Associated with Race and Ethnicity}

\section{Javier Lasa, Meryl Cohen, Gil Wernovsky, Nelangi Pinto \\ The Cardiac Center, The Children's Hospital of Philadelphia}

Background: Previous studies have explored the association of in-hospital mortality with race and ethnicity in neonates undergoing congenital heart surgery (CHS). However, little is known regarding the impact of race and ethnicity on morbidity and mortality after hospital discharge for this population. Methods: A retrospective chart review was performed of neonates $(<30$ days of age) who underwent CHS between January 2005 and June 2006. Exclusion criteria included death prior to discharge, isolated PDA ligation or vascular ring repair, and primary residence outside the United States. The association of race and ethnicity with mortality after hospital discharge was assessed in all patients. In addition, a cross-sectional telephone survey of families of surviving children was conducted to examine the association of race, ethnicity as well as social factors (primary language, education and income) with morbidity (unplanned hospital admissions or cardiac re-interventions). Data was analyzed using Fisher's exact, rank sum and multiple logistic regression. Results: Among 217 subjects who met inclusion criteria, overall mortality after discharge was $6.4 \%$. No statistically significant difference in mortality was observed between white and non-white cohorts $(9.3 \%$ vs. $3.7 \%$, $p=0.09)$. However, in patients with less severe forms of heart disease, race was independently associated with with late mortality and morbidity as assessed by the survey. The survey completion rate was $53 \%$ with significantly more whites completing the survey compared to non-whites ( $59 \%$ vs. $36 \%$; p $=0.01$ ). No differences were seen in outcomes with regard to other social factors. Lastly, surviving non-whites were more likely to express dissatisfaction with access to care. Conclusion: In addition to the known effects on early mortality, racial and ethnic disparities continue to influence morbidity and mortality following hospital discharge for CHS in neonates. Further investigation is needed to determine the causative factors and to begin efforts to eliminate such differences.

\section{Standardized Handoff Communication: Improving Patient Safety and Staff Satisfaction Every Time}

Andrea Torzone, Jodie Lantz, James Allard, Jana Newman, Karen Norton, Jennifer Lagrone, Stephanie Dickson, Frank Kern, Michael Spann, Jean Storey

\section{Children's Medical Center Dallas}

Background: Patient handoff is a critical period, but especially so for complex, critically ill patients in the pediatric cardiac intensive care unit (CICU). Evidence supports that errors occur when multiple disciplines and locations are involved in transfer; such handoffs are frequent in CICU. A Joint Commission National Patient Safety Goal is to improve the effectiveness of communication among caregivers, specifically hand-off communication. Nursing recognized the high-risk practice of nonstandardized communication between anesthesia and nursing during postoperative admission and identified an opportunity for process improvement. We hypothesized that a standardized OR to ICU Handoff Tool would improve communication, resulting in a safer patient environment postoperatively, and would also improve staff satisfaction with the transfer process. Methods: A dedicated nursing team envisioned a standardized communication tool for OR to CICU handoffs. Existing process was observed; gaps were identified. A checklist was drafted; initial review by nursing staff prompted discussion about the entire patient transfer process and necessary information for nurses to provide optimal care for these complex patients. The Handoff Tool was evaluated and revised by Heart Center Leadership, Nursing, Anesthesia, and Unit Council. Bedside nurses served as champions for 
this project and provided education to peers on the new OR handoff process. Emphasis was placed on improving critical thinking during the post-op admission process as well as utilizing the Tool for teaching new nurses. This collaborative effort raised multi-disciplinary awareness of the critical nature of patient handoffs from OR to ICU and encouraged medical and nursing ownership of their contributive part in the process. Results: A standard of practice now exists when transferring patients from cardiac OR to ICU. The standardized handoff tool has improved nursing preparedness for a child's postoperative arrival, identified critical information to be passed among teams, and decreased communication breaks between anesthesia, nursing, and medical staff. The OR to ICU Handoff Tool has been accepted and implemented by nurses, as an organizational and teaching tool. Nurses took ownership of this process improvement and are now actively involved in the continuum of patient care; we have moved from "reactive" to "proactive." The success of this initiative prompted plans to spread standardized handoff communication to all Heart Center patient transfers, including catheterization lab, Cardiology Ward, and Clinic. We are gathering metrics focused on patient safety and nurse satisfaction surrounding implementation of the tool. We hypothesize that initiation time of critical medications including sedation and anticoagulation has decreased, as has overall "settle in" period after patient arrival from the Operating Room. Anecdotally, nurses are thrilled about receiving more information about their patients and the ability to anticipate postop course. Conclusion: Our OR to CICU Handoff Communication tool has dramatically improved a high-risk patient process. Ensuring that relevant, current patient data is transferred to the receiving team has helped ensure patient safety by allowing adequate preparation time for patient arrival and immediate initiation of hemodynamic monitoring during this critical period. The nurse-driven creation and implementation of our tool has raised multi-disciplinary awareness of the importance of standard communication during patient handoff and heightened nurses' critical thinking regarding these complex patients' physiology and expected post-operative course.

\section{Variation in Models of Care Delivery for Patients Undergoing Congenital Heart Surgery: A National Survey}

Danielle Burstein, Anthony F. Rossi, Jeffrey Phillip Jacobs, Paul A. Checchia, Gil Wernovsky, Jennifer S. Li, Sara K. Pasquali

Duke University Medical Center, Miami Children's Hospital, All Children's Hospital/The Congenital
Heart Institute of Florida (CHIF), St. Louis Child Hosp, The Children's Hospital of Philadelphia

Background: There are limited data regarding contemporary models of post-operative care utilized for patients undergoing congenital heart surgery in the US. Defining current practice patterns is a necessary first step in evaluating outcomes associated with different models of care, and in developing subsequent quality improvement initiatives. The purpose of this study was to describe contemporary models of care delivery utilized by US centers for patients undergoing congenital heart surgery. Methods: A cross-sectional evaluation of US centers caring for patients undergoing congenital heart surgery was performed using an internet-based survey. Data regarding center characteristics, and the setting and personnel involved in post-operative care were collected and described overall, and compared between centers providing care in a general pediatric intensive care unit (PICU) vs. dedicated pediatric cardiac intensive care unit (CICU). Results: A total of 94 (77\%) of the estimated 122 US centers caring for patients undergoing congenital heart surgery participated in the survey. The majority (79\%) of centers were affiliated with a university. Approximately half were located in a free standing children's hospital, and half in a children's hospital in a hospital. Fifty-five percent provided care in a PICU vs. $45 \%$ in a CICU. Physicians responsible for postoperative care included a combination of cardiology and/or critical care $(47 \%)$, critical care only without involvement of cardiology (39\%), cardiothoracic surgery $(32 \%)$, physicians with dual training in cardiology/critical care (15\%), and cardiology only without involvement of critical care $(9 \%)$. In the majority of cases, more than one of these physician groups was involved in postoperative care. Regarding surgical care, the majority of centers $(72 \%)$ reported that their pediatric cardiovascular surgeons also operate on adults with congenital heart disease. Trainee involvement in care most often included critical care fellows (53\%), pediatric residents $(53 \%)$, and cardiology fellows (47\%). Most centers (76\%) also utilized physician extenders. In centers with a CICU, there was greater involvement in post-operative care of cardiologists and physicians with dual training (cardiology and critical care), fellows vs. residents, and physician extenders. Conclusion: Substantial variation exists in current models of care delivery utilized for patients undergoing congenital heart surgery in the US. Further study is necessary to evaluate the implications of this variability on patient outcomes. 
Improving Care and Changing Culture through Nursing Developmental Rounds

Amy Jo Lisanti, Jean Marie Carroll, Barbara Medoff-Cooper, Jessica Patrell, J.-S. Rim, Donna Rust

\section{The Cardiac Center, The Children's Hospital of Philadelphia}

Background: Individualized, family-centered, developmental care (IFDC) is an established care model for medically fragile infants in neonatal intensive care units across the globe. The literature is replete with evidence about the benefits of IFDC practices. No literature exists on the use of IFDC in infants with congenital heart disease (CHD). However, these infants can benefit from IFDC interventions such as containment positioning, cue-based care, and procedural sucrose. The Cardiac Center at The Children's Hospital of Philadelphia provides state of the art care to medically fragile infants with congenital heart disease (CHD). Although IFDC practices are included in the hospital's nursing standards, they were not an established standard of care in CHD patients and were not being routinely used by the nursing staff. Cardiac Center nurses took the first steps towards changing the culture of care for infants with CHD by improving the awareness and practice of IFDC at the bedside. They started a Developmental Care Work Group (DCWG) and implemented weekly nursing developmental rounds. Methods: DCWG members established a monthly meeting and journal club in February, 2009. They reviewed current literature related to IFDC practices. IFDC is described as a philosophy and culture of care. Its central attributes include individualization of care, promotion of social and physical interaction between infant and parent, and allowance for flexibility as the process of care evolves throughout time. IFDC aims to empower families to participate in care to promote bonding and parental-infant attachment. Based on these essential attributes, the DCWG created a Developmental Rounding Tool (DRT) to promote individualized care of infants in the Cardiac Center and sensitize caregivers to assess and respond to infants' behavioral cues. The DCWG narrowed the scope of rounds to address pain management for infants less than six months of age. Results: Weekly rounds were successfully established in the Cardiac Center. Each Monday, three to five patients are identified within the Cardiac Center and DCWG members perform bedside rounds for approximately ninety minutes. During rounds, the infant's parents, when present, and the bedside nurse engage in an informal discussion with DCWG members about the infant's status and developmental issues. The DRT is completed by the bedside nurse or parent and recommendations are given to address the unique needs of each infant. The DRT is posted at the infant's bedside so that all caregivers can view, follow, and adjust recommendations as the neonate's needs change.

Patients are identified for child life, lactation, physical, occupational, and/or speech consults. Midline, flexed, and containment positioning are frequently reinforced. Non-pharmacologic comfort measures are encouraged and posted on the DRT. Parents have been eager to participate and use the rounds to share their experiences and concerns. After bedside rounds, the group debriefs to discuss the process of rounds, reflect on issues, and address trends. Conclusion: Many of the infants in the cardiac center now have a DRT at their bedsides and IFDC practices are being integrated into infants' care. The first steps have been taken towards the ultimate goal of fostering an interprofessional, collaborative culture that supports IFDC.

\section{Key Components of a Successful Interstage Home Monitoring Program for Infants Following Modified Norwood Operation}

Kaberi Mozumder, Jennifer Bond, Dannielle Toner, Carley Goldberg, Tara Drake, Laura Montafi, Jana Norris, Guadalupe Tovar, Frank Hanley, V. Mohan Reddy, Gail Wright

\section{Lucile Packard Children's Hospital at Stanford}

Background: Infants undergoing Norwood palliation historically had $7-15 \%$ interstage mortality. From 2002 to 2005, interstage mortality after the modified Norwood operation was $7 \%$ in our program. Since initiation of an interstage home monitoring program (HMP) in 2005, all Norwood patients have survived the interstage period. Our HMP team reviewed our results and lessons learned during program initiation and maintenance, which have contributed to successful outcomes. Key components of our HMP may aid in implementation of similar programs at other centers. Methods: Our HMP multidisciplinary team consists of pediatric nurse practitioners (PNPs), physician assistants, a nurse case manager, and a supporting cardiologist. We retrospectively reviewed HMP cases from the past 4 years to assess interstage survival, interventions, and admissions, as well as care coordination, communication, barriers to care, equipment issues, and program staffing. Additionally, we reviewed adjustments made to the HMP and evaluated how these changes contributed to the success of the program. All infants who survived to hospital discharge following Norwood 
operation were enrolled in the interstage HMP, irrespective of primary language, outpatient cardiologist, or proximity to our hospital. Interstage was defined as the time between Norwood hospital discharge and admission for Glenn operation. Results: From October 2005 to October 2009, 38 Norwood patients have been discharged on the HMP. There has been $100 \%$ enrollment and $100 \%$ interstage survival. The HMP altered clinical management in $40 \%$ of patients, resulting in admission and timely intervention. NonEnglish speaking families and those who lived more than an hour away were identified as the most at risk for gaps in care and readmission. The program has been received well by referring cardiologists and families. Essential components of our HMP were identified: 1) family teaching and equipment ordering initiated early in the intensive care unit, 2) families given a binder of resources, including warning signs, emergency procedures, and standardized sheets for documentation, 3) communication initiated between the HMP and the primary cardiologist and pediatrician, and outpatient appointments scheduled prior to discharge, 4) catheterization and Glenn surgery dates scheduled prior to discharge, 5) digital scales and pulse oximeters provided, and oxygen saturation, heart rate, weight, and feedings documented daily at home by families, 6) primary PNP designated for each family, 7) Spanish speaking PNPs assigned to Spanish speaking families and written instructions provided in Spanish, 8) families called at least weekly by PNPs to evaluate trends in monitored parameters, and families encouraged to call the team with any concerns, 9) patients with any concerns called more frequently, scheduled for a clinic appointment with their cardiologist, or admitted, 10) readmissions directed to the PNP service, 11) HMP supervised by a single cardiologist, 12) frequent communication maintained with community cardiologists. Conclusion: Implementation of an interstage HMP reduces interstage mortality in Norwood patients. We identified key components of our HMP. PNPs can play a leading role in comprehensive care coordination and continuity of care, which are important to HMP success. Further research and collaboration may facilitate implementation of similar programs at other institutions.

\section{The Impact of an Intensivist-Directed Multidisciplinary Pediatric Cardiac Critical Care Model on Outcomes Following Cardiac Surgery}

Jennifer L. Helman, Erin D. Mayo, Alexandre T. Rotta

\author{
Pediatric Cardiac Critical Care Program, Riley Hospital \\ for Children, Indiana University
}

Background: Several studies support an association between Intensive Care Unit organizational or staffing models and outcomes in critically ill adult patients. However, little is known about whether or not outcomes can be affected by the structure of a team caring for children and young adults following surgery to repair or palliate congenital heart defects. We hypothesized that an intensivist-directed cooperative model with a dedicated multidisciplinary Pediatric Cardiac Critical Care team would be associated with improved outcomes following cardiac surgery. Methods: On January 1, 2009, care at our institution transitioned from a surgeon-led team with physician extenders and on-demand, but not necessarily in loco, multispecialty support to an intensivist-directed cooperative dedicated multidisciplinary Pediatric Cardiac Critical Care Team. This multidisciplinary team includes pediatric nurse practitioners with extensive cardiovascular experience, pediatric intensivists, cardiologists, cardiology and critical care fellows, bedside nurses, respiratory therapist, pharmacist, nutritionist and social worker. Consultants from all relevant specialties are available as needed. The new model includes structured teaching and work rounds twice daily, organized and open communication between the critical care team and the surgeons, as well as the continued presence of an intensivist and pediatric nurse practitioners in the unit. We retrospectively reviewed clinical data from every patient who required critical care following cardiac surgery in 2008 (reflective of the previous care model) and for the first 8 months of 2009 (reflective of the new care model). Outcomes derived from continuous data were analyzed with the t-test or the Mann-Whitney Rank Sum test, while categorical data were analyzed in contingency tables to determine Risk Ratios (RR) and confidence intervals (CI). Values are reported as means \pm standard deviations, unless otherwise noted. Results: In 2008, 334 procedures were performed in 317 patients subsequently admitted to the Intensive Care Unit, averaging 27.8 cases per month. In the first 8 months of 2009, 212 procedures were performed in 204 patients averaging 26.5 cases per month. These numbers exclude patients that did not require admission to the PICU after surgery. The new care model was associated with a trend in shorter duration of mechanical ventilation $(4[2-15]$ vs. $5[2-11]$ days, $\mathrm{p}=0.09$, values are medians and 25-75th\%ile), a trend in reduced ICU length of stay $(5.65 \pm 9.36$ days vs. $7.32 \pm 15.67, \mathrm{p}=0.54)$ and a significantly lower length of stay index (actual/expected hospital length of stay) $(0.88 \pm 0.72$ vs. $1.01 \pm 0.78$, $\mathrm{p}=0.005)$ compared to the previous care model. The new care model was also associated with strong 
trends in decreased 30 day mortality $(1.42 \%$ vs. $3.15 \%$, RR 0.47 , 95\%CI 0.12-1.58) and hospital mortality $(1.42 \%$ vs. $3.89 \%$, RR $0.26,95 \% \mathrm{CI}$ 0.1-1.26) compared to the previous care model, despite a significantly higher severity of illness (3.3 \pm 0.79 vs. $3.1 \pm 0.91, p=0.022)$, respectively. Conclusion: An intensivist-directed cooperative model with a dedicated multidisciplinary Pediatric Cardiac Critical Care team appears to be associated with improved outcomes in children and young adults who require critical care following cardiac surgery.

\section{Adherence to AHA guidelines for Cardiovascular Risk Reduction in High Risk Pediatric Patients: Review of Cardiology and Endocrinology Practices}

Corey Stiver, Jennifer Dyer, Bethany Hashiguchi, Rebecca Cohen, Rebecca Anderson, Kerry Rosen

\section{Nationwide Children's Hospital}

Background: In 2006, the American Heart Association (AHA) published guidelines, endorsed by the American Academy of Pediatrics (AAP), to address the reduction of early coronary artery disease in high risk pediatric patients. Awareness of these recommendations may be limited for the general practitioner and sub-specialist. The purpose of this study was to review documentation of cardiology and endocrinology sub-specialty provider's recommendations to obtain fasting lipid panel and glucose, and diet and exercise prescriptions in patients considered to have increased risk of early coronary artery disease. Methods: Retrospective outpatient chart review of cardiology and endocrinology patients, ages 2-18 years, with diagnoses considered to have increased risk of coronary artery disease at a single institution from January to December 2007. Cardiology patients included Kawasaki disease, left heart obstructive lesions, hypertrophic cardiomyopathy, heart transplant recipients and patients with coronary manipulation, e.g. transposition of the great arteries arterial switch patients. Endocrine patients included patients with type I and II diabetes mellitus. Documentation of provider's recommendations to obtain fasting lipid panel, fasting glucose, $\mathrm{HgA1C}$ for diabetic patients, and diet and exercise prescriptions was assessed. Patients with multiple visits in one year were reviewed once. Logistic regression analysis was performed to assess age as a predictor of recommending lipid panel. Results: 407 cardiology patient encounters and 995 diabetic patient encounters, totaling 1402 eligible patients were reviewed. Mean age was 11.2 years $(+/-4.58)$. $45 \%$ were female. $86 \%$ of diabetic patients were type I. Fasting lipid panel was recommended in $31 \%$ of the diabetic patients and $0 \%$ of the cardiology patients. Fasting glucose was recommended in $0 \%$ of cardiology patients. $\mathrm{HgAlC}$ measurement was recommended in $99 \%$ of diabetic patients. Frequency of documented lifestyle modification recommendations in endocrine/diabetic patients: diet changes discussed $91 \%$, nutrition referral $86 \%$, exercise instructions $49 \%$; documented recommendations in cardiology patients: diet $5 \%$, nutrition referral $1 \%$, exercise $5 \%$. In the endocrine cohort logistic regression analysis revealed the likelihood of recommendation of lipid panel increased $30 \%$ per year of age, odds ratio: 1.29, $\mathrm{p}<0.05$. Conclusion: Increased risk of early coronary artery disease is recognized in certain pediatric patients including those with diabetes mellitus and some forms of congenital or acquired heart disease. Awareness of AHA and AAP endorsed guidelines to assess lipid panel/fasting glucose and lifestyle modification recommendations is lacking. Diabetic patients were more likely to have lipid panel and lifestyle modification recommendations made compared to cardiology patients. Likelihood of lipid screening recommendations in diabetic patients increased $30 \%$ per each year of life. Increased awareness and adherence to the guidelines may decrease the development of early coronary artery disease in high risk pediatric patients.

\section{Share Our World: A Unique Opportunity for Cardiac Nurses to Share Their Work and Experiences with Family Members and Loved Ones}

Amanda Seelhorst, Linda Goss, Kevin McNally, Tara Pritchett, Michele Romaniello, Marilyn Blaustein, Donna Rust, Meredith McDonnough, Elizabeth Steinmiller, Kim Delaney, Amy Lisanti

\section{The Cardiac Center, The Children's Hospital of Philadelphia}

Background: Historically, nurses in the Cardiac Center reported frustration with their inability to describe their work to their family and loved ones. Nurses stated, "My family doesn't understand why I am not able to leave on time", "My Mom thinks that I come to work and watch babies sleep all night and wonders how that could be stressful." Nurses stated they would like to show their family members what they do so they would understand and better support their work. Knowing that work life balance and personal support are key factors in staff retention and satisfaction, a team of leadership and staff nurses came together to create such an 
opportunity. Methods: The planning team decided to hold two events to give staff nurses the opportunity to bring family members to the hospital to get an inside view of the experiences of working in pediatric cardiac care. These events were named Share Our World.

The goals of Share Our World were:

1. Provide education to loved ones of nurses regarding the work and mission of Cardiac Center Nurses

2. Express appreciation to Cardiac Center nurses for their hard work, dedication, and compassionate care

3. Allow the opportunity for staff nurses to thank their family members for their support

The two events were held in Spring 2009. The entire nursing leadership team attended each event in order to meet and personally thank each nurse and their family members. The agenda included presentations from senior medical and nursing leadership in the Cardiac Center. An adolescent patient and parent also discussed their experiences in the Cardiac Center and what nurses have meant to them. The Nurse Manager recognized each nurse individually and presented personalized statements of appreciation from their direct supervisor and colleagues. The nurses then gave their family members a personalized thank you letter. Attendees enjoyed a light meal and concluded with a tour of the inpatient units. Results: All guests completed surveys at the end of each event. The staffs' loved ones expressed their gratitude for the opportunity to share in the experiences of their Cardiac Center Nurses. They also suggested that they would love the opportunity to attend again to learn more about how to support their nurses. One week following each event, the nurses who had attended completed an online survey. The results showed that nurses felt their family members had a better understanding for their work. They stated that their loved ones had been more understanding regarding the sometimes stressful nature of working as a pediatric cardiac nurse. Conclusion: The work of cardiac center nurses can be as stressful as it is rewarding. As we strive to provide family centered care for our patients, we must recognize the importance of loved ones as key to the success and support of nurses. If nurses feel supported, they will continue to provide excellent care.

\section{Prostaglandin Exposure and Operative Outcomes in Transposition of the Great Arteries with Intact Ventricular Septum}

Ryan Butts, Alexander Ellis, Scott Bradley, Thomas Hulsey, Andrew Atz

Medical University of South Carolina
Background: Neonates with D-Transposition of the Great Arteries with intact ventricular septum (TGA-IVS) often require infusion of prostaglandin (PGE) in order to maintain adequate systemic oxygen delivery prior to surgical correction. However, concern exists regarding the multiple side effects of PGE including apnea, fever, and increased risk of sepsis, as well as the possible detrimental effects on operative and postoperative outcomes. Methods: We performed a retrospective review of fifty-nine consecutive neonates with TGA-IVS undergoing arterial switch operation from 1995 to 2008 at our institution by a single surgeon. We sought to identify associations between perioperative outcomes and cumulative preoperative PGE exposure in hours. Primary outcomes included highest postoperative inotrope score, postoperative ventilator time, and length of postoperative ICU and hospital stay. Secondary outcomes measured included cardiopulmonary and cross-clamp times, minimum seventy-two hour postoperative blood $\mathrm{pH}$, and net postoperative fluid balance over the first 120 hours. Results: Our study population of 59 patients consisted of 40 males, 41 underwent preoperative balloon atrial septostomy. The median preoperative exposure to PGE was 32 hours, range zero to 176. At time of surgery, 29 patients had PGE infusing within the previous 12 hours. Eight patients were mechanically ventilated at time of surgery, all with persistent PGE infusion. There was no association between preoperative PGE hours and cardiopulmonary bypass time $(\mathrm{p}=0.91)$ and crossclamp time $(\mathrm{p}=0.78)$. Increasing cumulative preoperative PGE hours was associated with longer postoperative ventilator time $(\mathrm{p}=0.02)$ and lower minimum postoperative minimum $\mathrm{pH}(\mathrm{p}=0.02)$; however this did not result in longer postoperative ICU stay or postoperative total hospital days. There was a trend towards longer cumulative PGE exposure being associated with lower postoperative inotrope scores $(\mathrm{p}=0.08)$. Longer cumulative PGE exposure was associated with larger net negative fluid balance in the postoperative period ( $\mathrm{p}=0.02)$. Conclusion: Prolonged preoperative PGE exposure is not associated with intraoperative parameters of cardiopulmonary bypass time and cross-clamp time, nor postoperative length of ICU or hospital stay. Longer cumulative preoperative PGE exposure preoperative is associated with a longer postoperative ventilatory time and greater postoperative net negative fluid balance; likely a consequence of preoperative fluid retention from PGE. Prolonged preoperative PGE exposure may result in lower postoperative inotrope scores suggesting a potential benefit from ductal patency in maintaining preoperative left ventricle at systemic pressures. 
Pediatric Oral Health Care and Cardiac Surgery: Providing and Improving Assessment and Access

Martha Willis, Janene Allen, Lauren King, Peter Manning

\section{Cincinnati Children's Hospital Medical Center}

Background: Dental caries are the most common chronic childhood disease with a higher prevalence than asthma, hay fever and chronic bronchitis. Evidence has shown that children with congenital heart disease (CHD) have higher levels of untreated dental caries and are not receiving adequate preventative dental care. Cyanotic children have more actively decaying teeth and lower levels of treatment when compared with healthy controls. Chronic dental disease processes lead to an elevated systemic inflammatory host response, bacteremia, and endocarditis. Dental plaque provides a microhabitat for organisms that can translocate to and colonize other parts of the body, specifically the heart. Over the past few months 6 surgeries have been canceled at a Midwest children's hospital the day before surgery due to poor dentition and, thus, risk for potential complications following cardiac surgery. This has resulted in emotional, time, and financial expenditure for the patients, their families, and hospital staff. Lost work time, disruption to daily life, and the frustration of cancelled surgery along with the need to reschedule a surgery are extremely stressful situations for the patients and their families. As a result of cancelling surgery at such a late time and a literature review, we have implemented a dental care assessment and education program. The goal is to deliver comprehensive preoperative care. Methods: Upon realizing that children with CHD had surgery cancelled due to late diagnosis of dental decay and abcesses, we implemented a family and staff education program. The program provides comprehensive, high quality patient care through interdisciplinary team collaboration. Cooperation among the health care team and family is imperative to ensure preventative and restorative dental care and cardiac surgery occurs. The program began with development of a hand out for patients and their families that included information about oral care, dentists, and access. We then educated nursing staff in the cardiology clinic about the importance of dental care and added dental assessment to the history intake form. The cardiologists have also made it regular practice to address oral health during their visit. The patients and their families receive phone calls from the nurse practitioner two to four weeks prior to surgery to review dental care and the need for intervention. Results: Incorporating dental assessment and education in cardiology visits increases knowledge and awareness of the importance of dental care and cardiac defects. When educating families about oral health, patients can visit the dentist prior to cardiac surgery, care for their teeth, and avoid life threatening infections and cancellations or delays with cardiac surgery. Conclusion: Cardiac surgical delays due to dental decay are time, cost, and emotionally consuming. Regular and necessary dental care can facilitate high quality and prompt cardiac surgical care. Including dental care evaluation and encouraging oral health improves outcomes for children with cardiac defects.

\section{A Collaborative Effort To Prevent Skin Breakdown In Cardiothoracic Surgical Patients}

Sandy Conn, Bev Deem, Susan Putnam, Laurie Mustin

\section{Cincinnati Children's Hospital Medical Center}

Background: In January 2007, our organization began participating in a pressure ulcer prevalence study, and the results indicated that our rates of pressure ulcers as an organization were significantly higher than similar pediatric hospitals that we benchmarked against. Based on this knowledge, a task force within our organization developed an educational program that was designed to educate staff on in-depth skin assessments, identification of patients at risk, and appropriate interventions to decrease the risk of pressure ulcer development. Throughout the development of the educational program and subsequent pressure ulcer prevalence studies that occur quarterly it became clear that each unit would need a unit champion. The role of the unit champion is to reinforce education and to provide staff with additional bedside resources. Methods: Because of the education, increased awareness of staff, and the role of the unit champion, the bedside nurses started to notice that there were a significant number of patients that arrived to the CICU from the OR with reddened areas across the scapula, as well as various other areas of pressure points. The staff communicated their concerns and findings to the surgical team. With the help of the unit champion, a collaborative team was formed, which included nurses from the CICU and OR, a surgeon, APN, anesthesiologist, perfusionist, WOCN, legal consultant, and a representative from clinical and bioengineering. Each member 
contributed information related to their knowledge of the procedures necessary to perform care for the patient. An extensive review of literature was done by the WOCN, APN, and CICU nurses. Based on the work of the collaborative team, various practice changes in the OR were recommended with the hypothesis that no patients will develop pressure ulcers or skin breakdown in the OR. Results: Based on the recommendations from the team, the OR staff made immediate changes with patient positioning, and new OR mattresses were ordered. Another practice change included placing foam dressings on the patients back and sacrum to prevent pressure and breakdown to the skin. To better assess and evaluate the patient, the OR staff began rolling the patient to evaluate the skin upon transferring the patient from the OR table, and this information is now passed onto the CICU staff during safe hand off of care. With effective communication and commitment from each discipline to focus on patient well being and safety, there have been no further patients with skin breakdown or pressure ulcers on patients that were provided interventions prior to starting surgery. Conclusion: The care of our patients in the CICU does not occur in isolation of other departments, such as the cath lab and OR, etc., and we must work together if we want to continue to make a difference for our patients and families. We were excited to be part of a collaborative team that worked together, and continue to work together to make the changes that are necessary to prevent skin breakdown and pressure ulcers in our patients who undergo operative procedures in the OR.

\section{Validation of Continuous Cardiac Output Monitoring Based on Arterial Pressure Waveform in Pediatric Cardiac Transplant and Pulmonary Hypertension Patients: Preliminary Observations}

Sarena Teng, Jon Kaufman, Pan Zhaoxing, Angela Czaja, Heather Shockley, Eduardo daCruz

\section{The Children's Hospital, Denver; University of Colorado School of Medicine}

Background: Recently, a minimally invasive continuous cardiac output monitor based on arterial pressure waveform (APCO) was introduced (Flotrac/ Vigileo TM) and FDA approved for use in adults. The benefits of this device are 1) it does not require proprietary catheters- the sensor can be attached to any standard radial or femoral arterial catheter and 2) it does not require external calibration, rather it uses patient demographics and a proprietary algorithm to calculate cardiac output. There is no published literature validating this technology in pediatric patients. This study was designed to compare the APCO method with intermittent thermodilution via pulmonary artery catheter. Methods: Thirty-two patients scheduled for diagnostic cardiac catheterization, either for cardiac transplant follow up or for pulmonary hypertension evaluation were enrolled after IRB approval. Patients had pulmonary artery catheters (PAC) and arterial catheters placed as part of their routine care. Cardiac output (CO) measurements were obtained by ice cold bolus thermodilution, also as part of the patient's routine evaluation. Simultaneous CO measurements as determined by the Flotrac/Vigileo device were recorded. The Bland Altman method was used for statistical analysis and a percentage error of $30 \%$ or less was established as the criterion for method interchangeability. Results: A total of 32 pairs of $\mathrm{CO}$ measurements were obtained. Bland Altman plots for the means of all subjects with a body surface area (BSA) $\geqslant 1$ showed limits of agreement of -2.7 to 8.0 with no trend in variability dependent on the means of two measures. To examine whether the extent of agreement depends on BSA linear regression analysis was performed using percent change as a function of BSA. The linear regression analysis indicated that the accuracy increased with the BSA. To assess the precision of each measuring method, we analyzed the 1) coefficient of variance/ percent error and 2) the within-subjects variance. The coefficient of variance and percent error for the PAC were $37.6 \%$ and $75.37 \%$, respectively. The coefficient of variance and percent error for the Flotrac were $53 \%$ and $106 \%$, respectively. The intra-class correlation of the PAC was 0.929. The intra-class correlation of the Flotrac was 0.992. Conclusion: In this preliminary study, there was poor agreement between the Flotrac arterial pulse wave analysis and the PAC in determination of cardiac output in pediatric cardiac transplant and pulmonary hypertension patients. Although there is a growing body of literature validating the use of this technology in the adult surgical and medical patient, we did not observe the extent of agreement that has been reported in adults. There did seem to be a trend towards more agreement between these techniques with increasing BSA, and the intra-class correlation was better with the Flotrac. This may suggest that there was less variability in the sets of measurements obtained within a particular patient, and therefore more precision with the Flotrac method. The applicability of arterial contour pulse analysis in the pediatric cardiac population requires further investigation. 
Right Nurse for the Right Patient: Creation of the Cardiac Preparation and Recovery Unit

Donna Calfin, Lisa Brogan, Ashley Lebo, Lauren Stahl, Mary Stuart

\section{The Cardiac Center, The Children's Hospital of Philadelphia}

Background: Before the creation of the Cardiac Preparation and Recovery Unit (CPRU), all nonintensive care (ICU) post cardiac catheterization patients returned to the Cardiac Care Unit (CCU) following their procedure, while recovering from anesthesia or sedation. These patients included children with congenital or acquired heart defects, arrhythmias, pulmonary hypertension, and postacute heart transplant recipients. Patient care issues identified in the CCU included nurses trying to recover patients with additional patients in their assignments as well as trying to keep a large staff aware of sedation and recovery standards as policies changed. All care providers agreed these patients needed a specialized unit and staff to recover them in a safe and consistent manner. Arrangements were soon made to have non-ICU patients recover in an improvised "recovery room" located in a separate space, staffed by nurses from the CCU. This was the beginning of the CPRU. Methods: The CPRU began to follow the American Society of PeriAnesthesia Nurses (ASPAN) guidelines, which specify nurse to patient ratios. The guidelines are based on the anesthesia recovery system classifying patients into Phase I or II of recovery, depending on their level of consciousness. A competency based orientation process, ranging from four to six weeks, was developed to train experienced nurses in advanced airway management and post-anesthesia care of pediatric cardiac patients. The regimen includes working with a cardiac anesthesiologist, a respiratory therapist, and a general operating room (OR) Post-Anesthesia Care Unit (PACU) nurse for 2 days each, followed by a few weeks of orientation giving direct patient care in the CPRU under the supervision of a dedicated preceptor.

Currently, a core team of cardiac nurses trained in post-anesthesia care provide patients and families consistent caregivers, offering familiarity with flow through the cardiac center. All non-ICU patients who receive cardiac anesthesia/sedation or require monitoring by cardiac nurses for procedures, now recover in the CPRU for both cardiac and noncardiac procedures. Results: A cardiac pediatric recovery unit offers specialized care to a very specific and fragile population, who typically require frequent procedures with anesthetics or sedation. The unit also affords children and their families consistency of care from an interdisciplinary team of pediatric cardiac specialists. This consistency was reflected in recent CPRU and Press Ganey survey results, which showed positive results for patient/ family satisfaction. The CPRU received high scores in areas of patients receiving special attention; promptness to calls; information being relayed in a clear manner; nursing skill sets; sensitivity to pain; and family preparation for discharge. Conclusion: The CPRU has evolved into a dedicated 13 bed unit located near the Cardiac ORs and Cardiac Catheterization Labs. For the fiscal year 2008, the CPRU provided care to approximately 2300 patients including 900 cardiac catheterizations. The projected number of cases for the fiscal year 2010 is expected to reach approximately 3000. The CPRU now serves not only cardiac catheterization patients, but also cardiac patients who are undergoing other cardiac and non-cardiac procedures. The CPRU is clearly providing the right nurse for the right patient.

\section{Improving Patient Care in the PCICU by Increasing Nursing Participation Into Rounds: The Critical Steps Necessary for Success}

Christine Agee, Kyle Rehder, Jerome Chen, Jon Meliones

\section{Duke Children's Hospital and Health Center}

Background: Patient (Pt) care rounds provide a unique opportunity for the entire team to contribute to the development of a shared mental model for Pt care. The role of the nurses (RN) on daily rounds varies across institutions and can significantly impact the delivery of care as well as RN satisfaction. Little data exists on the optimal method to integrate RNs input into rounds that provides a seamless and satisfying presentation for all team members. In the past, RNs have expressed feelings of being disconnected from rounds and not having a clear understanding of the plan for the day. We hypothesized that in the Pediatric Cardiac Intensive Care Unit (PCICU), including RNs as an integral part of the presentation during rounds would increase RN satisfaction, increase knowledge of orders, and implementation of $\mathrm{Pt}$ goals. Methods: After observing interactions on daily rounds and conducting individual discussions with RN staff, several opportunities for improvement were identified. Common themes obstructing RN input during rounds were: being busy with Pt care, not feeling their concerns were valued, and not 
knowing what to say or what was expected. Based on these common themes daily $\mathrm{Pt}$ rounds were adjusted to include an $\mathrm{RN}$ input section. The RNs were then given clear expectations of what to review during this time. For example, RNs felt invasive lines/tubes were often misrepresented on rounds; this provided an opportunity for the RNs to be accountable for leading this discussion. Another roadblock to participation expressed by the RNs was not knowing when to provide their input into the presentation. The rounding sheets were therefore modified to include a prompt to the presenting clinician to ask the Pt's $\mathrm{RN}$ for their input at a consistent time in every presentation. Goals for the day and the orders that were entered were also repeated at the end of each presentation and confirmed by the RN. Results: Nursing input significantly increased after training $(44 \%$ to $100 \%, \mathrm{p}<0.05)$ as well as satisfaction with daily rounds $(33 \%$ to $94 \%, \mathrm{p}<0.05)$. Creating a shared mental model through reviewing orders and plans for the day was found to be critical in $100 \% \mathrm{RNs}$ and after training occurred in $94 \%$ of rounds. These efforts contributed to a $70 \%$ reduction in $\mathrm{RN}$ terminations from $24.7 \%$ in FY06 to $8 \%$ in FY09. As a byproduct of these interventions, patient flow has increased by $21 \%$. In addition, $96 \%$ of RNs would welcome increased participation in rounds. Conclusion: Including nursing input on rounds significantly increases their satisfaction and has a positive impact on the delivery of care. Formalization of this process should include; clearly defining the RN's role during the presentation, educating the RNs on structured communication methods, and educating the entire team on the value of developing a shared mental model.

\section{Iron Deficiency not Associated with Collateral formation in Single Ventricle Patients}

Jamie Penk, Konstantin Averin, Christine Sullivan, Dave Wax

\section{Children's Memorial Hospital}

Introduction: Single ventricle patients frequently form systemic to pulmonary collaterals that complicate the clinical course and surgical procedures of these patients. Collaterals may be formed in response to inadequate oxygen delivery to the tissues. Several studies suggest that mediators such as vascular endothelial growth factor, released in response to hypoxia, have a role in this process. Patients with single ventricles have hypoxia with poor oxygen delivery that would be further compromised by iron deficiency and relative anemia.
We hypothesized that single ventricle patients with relative iron deficiency anemia would have an increased incidence of systemic to pulmonary collaterals. Materials and Methods: A retrospective chart review was performed. A catheterization lab database was searched for patients who had coils placed. From this search, forty-seven single ventricle patients under the age of six years old were identified. Most were undergoing catheterization prior to a Glenn or Fontan procedure. A cardiovascular surgery database was searched and sixty-five single ventricle patients under the age of six years old who underwent a Glenn or Fontan procedure, but did not have collaterals occluded in the catheterization lab or during surgery, were identified. Data collected included age, diagnosis, pulse oximetry, hemoglobin, mean corpuscular volume (MCV), mean corpuscular hemoglobin concentration (MCHC), red cell distribution width (RDW) and number of previous sternotomies. Since iron studies were not routinely obtained, MCV, MCHC, RDW and hemoglobin were used as markers for relative iron deficiency. Multiple logistic regression modeling was used to determine if markers consistent with iron deficiency were associated with a higher incidence of collaterals. Univariate logistic regression modeling was used to examine other variables associated with collateral formation. Results: There was no significant difference found between groups for MCV, MCHC, RDW or hemoglobin while controlling for pulse ox, age and number of sternotomies. Collateral formation was significantly associated with higher oxygen saturation $(\mathrm{p}=0.0002)$, increased number of sternotomies $(\mathrm{p}=0.0003)$ and age $(\mathrm{p}=0.014)$. Conclusion: There was no association found between iron deficiency and systemic to pulmonary collateral formation in young, single ventricle, patients. There is an association between collateral formation and age, higher oxygen saturation and also with number of sternotomies.

\section{Self Reported Health Status and Quality of Life in Men Operated for Tetralogy of Fallot Between 1971 and 1991}

Elisabeth Ellingsen, Lia Mendes Pedersen, Vibeke Elisabeth Hjortdal

\section{Aarbus University Hospital, Skejby, Aarbus, Denmark}

Background: Tetralogy of Fallot (TOF) has been treated successfully by surgery for decades. Gradually, age at surgery has decreased and long-term outcomes after surgery have become better. Today, long-term survival rates are over $95 \%$, and children operated for TOF has a quality of life similar to 
others at their own age. We have previously demonstrated that in women operated for TOF in the period 1972-1992, fertility is normal, pregnancy well tolerated and neonatal outcome excellent and that the women have a good health status. The recurrence risk of congenital cardiac disease, most often TOF, is high. The question that remains, and that we now address, is whether men with TOF are functioning at an equally high level. Methods: 66 men operated for TOF in Aarhus University Hospital in the period from 1971 through 1991 received a questionnaire containing the SF-36 health survey and questions about children, education and work status. Mean age Oct. 12009 was 34.2 (range 18-59) years. Thirty three healthy, age matched males will be used as control subjects. Results: Out of the 66 patients, eight $(12.1 \%)$ were operated at an age of $<2$ years, $29(43.9 \%)$ between two and four years, 26 (39.4\%) between five and 15 years, and three $(4.6 \%)$ after the age of 15 . Results from 48 patients, show that patients score excellent and similar to healthy, age matched control subjects, when they evaluate their level of bodily pain, vitality, social functioning, mental health and emotional status. Patients score 9.3\% ( $\mathrm{p}=0.002)$ lower than controls in physical functioning and $12.1 \%(\mathrm{p}=0.03)$ lower in role-physical functioning. There is also a tendency towards a lower general health score $(12.9 \%$ lower than controls $(\mathrm{p}=0.06))$. The general health score reveals how the responders look at their over-all health status, and their expectations concerning their health in the future. Sixteen $(33.3 \%)$ of these 48 men have received altogether 34 children. One child has a congenital heart disease. Thirty men $(62.5 \%)$ are currently working, while six $(12.5 \%)$ are studying. Ten $(20.8 \%)$ are either retired, receiving social security or in rehabilitation, while two $(4.2 \%)$ are unemployed. Seventeen $(35.4 \%)$ have completed a higher education and, $13(27.1 \%)$ have a craft certificate. Conclusion: The majority of men operated for tetralogy of Fallot in Aarhus, Denmark in the seventies and the eighties are generally doing well in terms of education, job, family and children. They consider their overall quality of life fine except for physical functioning and role-physical functioning. They also seem to have more concerns about their future health than normal men.

\section{Decreasing Morbidity in the PCICU Through Clinician Led Six Sigma Initiatives}

Christine Agee, Kyle Rehder, Jerome Chen, Jon Meliones

Duke Children's Hospital and Health Center
Background: Six Sigma's performance improvement (PI) methodology has been successful in healthcare areas with increased variability and lack of standardized care. Despite a decrease in mortality for complex congenital heart disease (CHD), patients in the Pediatric Cardiac Intensive Care Unit (PCICU) have high variability in their care leading to an increased length of stay (LOS). Increased LOS has been associated with increased morbidity related to prolonged mechanical ventilation, increased risk for nosocomial infection, etc. Our approach in indentifying practice areas with increased variability with a significant impact on LOS was to utilize the Six Sigma DMAIC methodology. Clinicians including attendings, nurse practitioners, fellows, and bedside care providers are important in identifying the potential root cause of the problem being addressed as well as implementing the changes. Methods: We prospectively collected data on 400 cardiac surgical patients for $2008(n=214)$ and $2009(n=186)$ utilizing the CardioAccess database in addition to a customized beside measurement tool that bedside nurses used to collect key postoperative data. Statistical analysis was conducted utilizing JMP software (SAS, Inc). Our 2008 analysis identified several opportunities with high variability such as; catheter associated blood stream infections (CA-BSI) specifically with variability in practice with maintenance bundles, variability in inotrope usage within similar diagnostic categories, and length of ventilation (LOV). Several initiatives were implemented including: a CA-BSI six sigma project, standardization of inotrope usage, respiratory management guidelines, as well emphasis on standardizing daily patient care rounds to develop a consensus for goals for the day. Results: Despite the fact no differences were identified in complexity of the patient population evidenced by the comprehensive Aristotle scores from 2009 and 2008 (mean 9.8 vs. 10.0, $\mathrm{p}=\mathrm{ns})$ a significant reduction in postoperative LOS was achieved in 2009 vs. 2008 (mean: 7.6 vs. 10.2, $\mathrm{p}<0.05$ ). Six Sigma efforts resulted in several significant changes in practice with a reduction in the use of dopamine and epinephrine in 2009 (2\% vs. $61 \%$ and 32 vs. $53 \% \mathrm{p}<0.05$, respectively), reduction in CA-BSI (5.8 to $1.1, \mathrm{p}<0.05$ ), and reduction in LOV (mean 4.7 vs. 7.7, p $<0.05$ ). Conclusion: Significant improvement in mortality have been achieved in complex CHD patients, the focus in care is now on reducing morbidity. Methodologies such as Six Sigma which focus on reducing variability and standardized care can lead to successful outcomes in reducing morbidity for these patients. Clinicians are ideally suited to lead these projects due to their insight and expertise in 
the variability in patient care practices and their ability to drive data driven initiatives leading to best practices in patient care.

\section{High Shear Congenital Cardiac Defects are associated with Platelet Function Defects in Children}

David K. Bailly, Vanessa J.C. Rogers, Julie Martchenke, Stephen M. Langley, Lynn Boshkov

\section{Oregon Health and Science University}

Background: Platelet function defects have been found to be associated with aortic valve pathology in adults due to increased shearing forces. High Molecular Weight von Willebrand Multimers necessary for the adherence of platelets are thought to be consumed at an increased rate in the presence of high shear forces resulting in Acquired Type IIb von Willebrand Disease. Abnormal shear forces are characteristic of many congenital cardiac lesions. The aim of the current study was to determine whether high shear congenital cardiac lesions are associated with an abnormal Platelet Function Analysis (PFA) in children. Methods: The PFA-100 test was used to preoperatively evaluate 250 consecutive patients between the ages of 1 month and 18 years who underwent cardiac surgery at our institution between February 2005 and April 2007. Patients with abnormal renal or liver function, hematocrit $<28$ or platelets $<100$ were excluded as were patients who had, or may have consumed aspirin or NSAIDs within 7 days of surgery. Cardiac defects were classified as high or low shear lesions. Right or Left outflow tract obstruction, Coarctation, Ventricular Septal Defect, Tetralogy of Fallot and patients awaiting Glenn procedures were classified as high shear lesions. Low shear lesions were considered to be Atrial Septal Defects, Pulmonary Regurgitation and those undergoing Fontan procedures without a concomitant high shear lesion. Other lesions, including AV canal defects, were considered too complex to be classified as involving high or low shear forces. Results: Of the 250 patients, 52 were excluded due to the absence of a preoperative PFA value. 37 patients were excluded due to aforementioned criteria. Data were analyzed for 161 patients, 105 (65.23\%) had abnormal PFAs, 56 $(34.78 \%)$ had normal PFAs. 103 were classified as high shear lesions, 34 as low and 24 others. Of the high shear lesions $78.64 \%$ (81) had abnormal PFA values as opposed to $35.29 \%$ (12) of patients with low shear lesions. The 24 patients with 'other' lesions were equally split between abnormal and normal PFA values. Conclusions: Platelet Function Defects are prevalent in patients with congenital cardiac defects.
A greater proportion of patients with high shear cardiac lesions have abnormal PFA values compared to those with low shear lesions. Further studies are needed to determine the clinical significance of these findings.

\section{Prepregnancy BMI and Congenital Heart Defects: A Population-based Study}

Nicolas L. Madsen, Stephen M. Schwartz, Mark B. Lewin, Beth A. Mueller

\section{Seattle Children's Hospital, University of Washington School of Public health}

Background: Most cases of congenital heart defects (CHD) do not have an appreciable genetic etiology. Therefore, certain environmental exposures have been postulated as responsible. We aimed to describe the association of infant CHD and maternal prepregnancy weight. Methods: We conducted a population-based case-control study of CHD using linked birth-hospital discharge records for Washington State during 1992-2007. All infants with CHD were identified by screening ICD codes of hospital records $(\mathrm{N}=11,263)$. For comparison, controls $(\mathrm{N}=140,470)$ were randomly selected from all live singleton births occurring during the same years. Maternal body mass index (BMI, $\mathrm{Kg} / \mathrm{m}^{2}$ ) was calculated from maternal prepregnancy data found in birth certificates, or via linkage to drivers' license records. Infants with chromosomal anomalies, premature infants with isolated Patent Ductus Arteriosus, and maternal established diabetics were excluded. Mantel-Haenszel stratified analysis was used to calculate adjusted odds ratios (OR) and 95\% confidence intervals (CI) for the association of CHD relative to maternal obesity $(\mathrm{BMI}>30)$ or overweight (BMI 25.0-29.9). Results: Infants with CHD were more likely to have an obese (OR: 1.22; 95\% CI: 1.15-1.30) mother (adjusted for gestational diabetes). The association with obesity was greatest for defects of Left or Right Ventricular Outflow Tract Obstructions (OR: 1.27; 95\% CI: 1.02-1.59 \& OR: 1.43; 95\% CI 1.20-1.69, respectively). Hypoplastic Left Heart Syndrome (HLHS) and Shone's type defects were particularly strongly associated (OR: 1.86; 95\% CI: 1.13-3.05 \& OR: 3.17; 95\% CI 0.96-10.48, respectively). There was no association measured when considering Conotruncal defects (OR: 1.04; 95\% CI: $0.82-1.33$ ) or Septal Defects (OR: 1.06; 95\% CI: 0.96-1.16). Conclusions: Our study confirmed the previously described association between CHD and maternal obesity. In addition, secondary to the large number of cases included, our study was able to convincingly demonstrate that selected subtypes of 
CHD, such as HLHS, appear to be most strongly associated. A greater risk of CHD among offspring is yet another adverse outcome associated with maternal obesity, and emphasizes a need for efforts to decrease levels of obesity among women.

\section{Outcomes of Infants $\leqslant 3 \mathrm{~kg}$ Requiring ECMO Support Following Cardiac Surgery}

Priya Bhat, Jennifer Hirsch, Sarah Gelehrter, Elaine Cooley, Janet Donohue, Karen King, James Gurney, Robert Gajarski

\section{University of Michigan Health System}

Background/Objective: Extracorporeal membrane oxygenation (ECMO) is the most frequently used form of mechanical cardiopulmonary support in pediatric patients following cardiac surgery. Cited survival for post-cardiotomy neonates has been $\sim 40 \%$. However, a recent review found that survival among neonates $<3 \mathrm{~kg}$ with hypoplastic left heart syndrome following Norwood palliation was $0 \%$ prompting this study to evaluate survival of post-cardiotomy infants weighing $\leqslant 3 \mathrm{~kg}$ requiring ECMO following cardiac surgery and to identify risk factors associated with poor outcome. Methods: A retrospective review (1/1/99-12/31/08) of our instititutional database was performed in infants $\leqslant 3 \mathrm{~kg}$ who required post-operative ECMO support following cardiac surgery. The primary outcome was survival to hospital discharge. Additional data included: weight, age, gestational age, age at ECMO initiation, cardiac diagnoses and RACHS-1 category, single or biventricular repair, cardiopulmonary bypass and circulatory arrest times, total ECMO support time, inotrope score and serum lactate level prior to and during ECMO support, and need for hemofiltration. Results: We identified 62 patients $\leqslant 3 \mathrm{~kg}$ requiring post-operative cardiac ECMO. The median age at ECMO initiation was 7 days (range: $1-77$ days), median weight was $2.6 \mathrm{~kg}$ (range: $1.3-3.0 \mathrm{~kg}$ ) and median gestational age was 37 weeks (range: 28-41 weeks). The median ECMO support time was 168 hours (range: 19-684 hours). Overall survival to hospital discharge was $27.4 \%(17 / 62)$ with median support times in survivors vs. non-survivors of 106 and 212 hours, respectively $(\mathrm{p}=0.04)$. The need for hemofiltration during ECMO was associated with poor survival (12\% survival among those requiring hemofiltration vs. $46 \%$ for those who did not; $\mathrm{p}=0.002$ ). Post-ECMO decannulation, survival was higher in patients with vs. those without renal failure requiring dialysis $(70 \%$ vs. $27.3 \% ; \mathrm{p}=0.03)$. Comparatively, inotrope scores (IS) were lower in survivors vs. non-survivors of 12.0 vs. 17.0 $(\mathrm{p}=0.06)$ and 11.5 vs. $16.7(\mathrm{p}=0.04)$ at 48 and
72 hours, respectively. The change in IS from the preECMO baseline among survivors vs. non-survivors was $-40 \%$ vs. $+10 \%(\mathrm{p}=0.01)$ and $-40 \%$ vs. $+12 \%$ $(\mathrm{p}=0.03)$ at 48 and 72 hours, respectively. Similarly, serum lactate levels were lower in survivors vs. nonsurvivors of 6.3 vs. $9.0 \mathrm{mmol} / \mathrm{L}(\mathrm{p}=0.04)$ and 4.3 vs. $8.7 \mathrm{mmol} / \mathrm{L}(\mathrm{p}=0.02)$ pre-ECMO and at 6 hours post-ECMO. Survival in neonates weighing $<2.5 \mathrm{~kg}$ was lower than those $2.5-3 \mathrm{~kg}(17 \%$ vs. $36 \%$; $\mathrm{p}=0.09$ ). Factors not associated with increased mortality included RACHS-1 score, single versus biventricular anatomy, cardiopulmonary bypass times, gestational age $<35$ weeks and appropriateness of weight for gestational age. Conclusions: Survival following cardiac ECMO for infants weighing $\leqslant 3 \mathrm{~kg}$ is poor. Factors associated with poor prognosis include a need for hemofiltration or post-ECMO dialysis, inability to wean inotrope support within 48 hours of ECMO initiation, longer duration on ECMO and higher serum lactate before and up to 6 hours on ECMO. These findings should help guide medical decision making for this high-risk, unique group of ECMO patients.

\section{Reducing Child and Parental Procedural Stress: Enhancement of a Pre Cardiac Catheterization Program}

Holly Pfriem, Janene Allen, Melissa Henson, Jamie Piening, Nancy Pulsar

\section{Cincinnati Children's Hospital Medical Center}

Background: Cardiac catheterization is often the first invasive procedure where the child is developmentally aware given advances in surgery and interventional catheterization during infancy. Assumptions are often made that a child and parent have a comprehensive understanding of the procedure due to their prior experiences. In reality, the cardiac catheterization can be emotionally challenging for the child and parents. It is well documented that procedural anxiety and stress can elicit negative behaviors and impair a child's ability to cope and recover. The greater the child's anxiety ultimately reflects the degree of stress experienced by the parents. As a result of evaluating our current practice and a literature search we implemented a developmentally and educationally appropriate Pre Cardiac Catheterization Program emphasizing a comprehensive individualized approach of the catheterizaton experience. Our goal was to decrease the level of anxiety and stress experienced by the child and the parents by providing a thorough individualized educational program thus promoting a positive hospital experience. Methods: Our Pre Cardiac Catheterization Program utilizes a multidisciplinary approach including a Nurse Practitioner (NP) and Child 
Life Specialist (CLS). With this approach we provide comprehensive medical and psychological preparation of the cardiac catheterization process involving both the child and parents. The program is adjusted to accommodate the developmental stage of the child and the identified learning needs and preferences of the child and parents. The role of the NP includes performing a history and physical and education about diagnosis, procedure including hands on device demonstration, hospital stay and recovery. The NP provides continuity to the child and parents by providing care at the pretesting visit, day of procedure and through discharge. The role of the CLS is to engage the child in age appropriate medical play, review a specifically designed Cath Lab photo album and Cath Lab Activity Book. The Cath Lab Activity Book allows the child to personalize their experience through coloring, drawing and journal writing. Tours include the Cath Lab, Recovery Room, hospital unit and child's bedroom. Cath Lab tours are offered bimonthly and upon request. An assessment of the child's developmental needs is completed by the NP and CLS. These observations are shared with the team to plan for therapeutic interventions that may be necessary throughout the catheterization process. Results: Enhancement of our Pre Cardiac Catheterization Program has allowed us to implement a comprehensive individualized program resulting in high quality patient and family centered education and care. Parents report that the individualized time spent with their child greatly decreased both their child's and their anxiety and led to a less threatening and stressful procedure. Parents and children reported overwhelming satisfaction during their catheterization experience. Conclusion: Incorporating the child's developmental stage and varied learning styles, our Pre Cardiac Catheterization Program provides a comprehensive, individualized educational preparation for children and their parents. The decrease in procedural stress and anxiety promotes a positive hospital experience and increases patient and parent satisfaction.

\section{Cost Containment Versus Quality of Care - Maintaining Standards and Efficiency}

Vanessa Garside, Elisabeth Smith, Teresa Johnson, Cho $\mathrm{Ng}$

Great Ormond Street Hospital for Children, London, United Kingdom

Background: There is an ongoing worldwide aim to continuously improve the efficiency of health care. Within the, UK there is an additional drive to improve efficiency while maximizing standards of care, provided in a government report by Lord
Darzi (2008). The role of the Nurse Practitioner in our institution is to place a positive impact on service improvements, cost containment, continuity/quality of care and advancing nursing practice. Methods: Since January 2006, we have successfully fast tracked 290 cardiac surgical patients. In 2008, out of 73 patients who were fast tracked, $72 \%$ were extubated in less than 1 hour and $80 \%$ of patients were discharged within 3 days, thereby reducing associated risk of Ventilator associated pneumonia (VAP), line sepsis and other nosocomial infections. Our aim was to bring about a potential change in practice by reducing the use of:

Post chest drain removal X-rays

Routine blood screens performed on day 2 .

12 lead ECG monitoring prior to pacing wire removal

A retrospective review was carried out of the last 40 patients with regards to these interventions with an aim to see if any abnormalities noted in these variables changed the course of clinical treatment or increased patient length of stay. Current evidence based practice in this area is limited, particularly in the paediatric arena. Results: Of this cohort, only 2 patients required a change in clinical treatment and extended hospital stay related to their chest X-ray. $60 \%$ of the group had no abnormalities associated with any of these variables and the remaining 35\% showed minor changes but these did not affect patient length of stay or result in a change to clinical treatment. Conclusion: We postulate, based on this data and the current pool of evidence based information, that these three procedures could be eliminated from the routine fast track care. These results suggest that standards of care and safety can be maintained with the support of thorough and appropriate use of excellent clinical assessment. Cost savings and efficiency are maintained by reducing invasive and costly procedures. Challenges to changes in practice remain between multiprofessional teams. We aim to address these concerns through the use of multiprofessional evidence based anticipated recovery pathways for cardiac fast track surgery, providing standardized care for this group of children, where the need for these types of investigations can be assessed and evaluated by ongoing audit under variance tracking.

\section{Global Perfusion Differences Exist Between Patients with Single Ventricle Anatomy Status Post Fontan Procedure When Compared to Normal Controls}

Jennifer E. Dixon, Rohit Rao, Michael Danduran

Children's Hospital of Wisconsin and Medical College of Wisconsin 
Background: Cardiopulmonary exercise testing (CPET) provides assessment of the integrative responses which are not adequately evaluated during resting conditions. Patients who have undergone Fontan modification secondary to single ventricle anatomy have exercise limitations. Near-infrared spectroscopy (NIRS) provides a non-invasive, continuous method to monitor regional tissue oxygenation $(\mathrm{rSO} 2)$ and provides a window into regional oxygen supply-demand relationships. Hypothesis: Multi-site NIRS monitoring will reveal unique desaturation patterns during CPET in patients with Fontan anatomy when compared to normal patients demonstrating differences in systemic oxygen consumption to flow-coupling dynamics observed during exercise. Methods: 50 normal and 36 Fontan individuals underwent maximal CPET with continuous NIRS monitoring (Somanetics Corporation, Troy MI) at the following sites: $\{\mathrm{rSO} 2 \mathrm{C}$ (Brain), $\mathrm{rSO} 2 \mathrm{R}$ (Kidney), rSO2 L (Leg) and rSO2 A (Arm)\}. rSO2 was recorded at rest, exercise, and through a five minute recovery period. Results: Group data is presented below with $(*=\mathrm{p}<0.01)$.

\begin{tabular}{lcc}
\hline & Normal & Fontan \\
\hline $\mathrm{N}$ & $50(25 \mathrm{~F}, 25 \mathrm{M})$ & $36(11 \mathrm{~F}, 25 \mathrm{M})$ \\
Age (years) & $15.4(7-21)$ & $11.6(5-23)$ \\
Peak HR (beats/min) & $194(164-210)$ & $166(117-200)^{*}$ \\
Endurance Time & $13: 09(9: 01-19: 15)$ & $9: 04(6: 00-12: 50)^{*}$ \\
VO2 (L/min) & $2.64(1.00-4.64)$ & $1.30(0.66-2.60)^{*}$ \\
VO2 \% Predicted & $102(56-140)$ & $67(47-110)^{*}$ \\
\hline
\end{tabular}

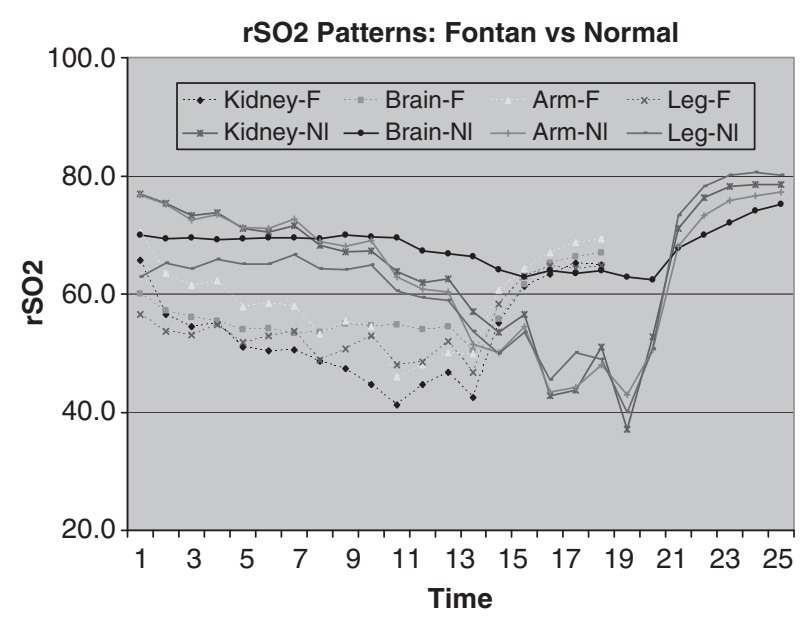

Graphical representation of NIRS data shows significant differences in Resting $\mathrm{rSO} 2-\mathrm{C}$, and $\mathrm{rSO} 2-\mathrm{R}$ at baseline. Differential saturation patterns are consistent throughout vascular beds with early desaturation noted in Fontan patients when compared to normal. Fontan patients have a steep initial slope of desaturation in cerebral circulation, while the post AT slope is lower than for normals with a larger rebound after exercise in the cerebral bed. DISCUSSION: Reduced exercise capacity in Fontan patients may be secondary to impaired cerebral auto regulation secondary to low systemic venous compliance.

\section{Sildenafil is Well Tolerated in Children and Young Adults Late After the Fontan Operation}

Nicole Mirarchi, David J. Goldberg, Andrew C. Glatz, Bradley S. Marino, Allison M. Paisley, Stephen M. Paridon, Michael G. McBride, Brian D. Hanna, Gil Wernovsky, Jack Rychik

\section{The Cardiac Center, The Children's Hospital of Pbiladelphia}

Background: Sildenafil may have an important role in the treatment of children and young adults with single ventricle physiology after the Fontan operation. In adults the most common side effects include: headache (16\%), flushing (10\%), and dyspepsia $(7 \%)$. There have also been isolated reports of sudden loss of vision and hearing. However, there are no data on the side effect profile of Sildenafil in the single ventricle population. Methods: We performed a trial evaluating the effect of Sildenafil compared to placebo on ventricular performance and exercise tolerance in children and young adults $11.3 \pm 3.8$ years after the Fontan operation. Subjects were randomly assigned to both Sildenafil (20 mg tid) and placebo for 6 weeks each with a six-week washout period between study phases. Subjects were contacted at minimum every 10 days and asked to report new and ongoing adverse events. Events were documented on deidentified report forms and graded as mild, moderate or severe based on institutional IRB guidelines. Causality of events was determined by the study investigators and reviewed by the study's medical safety officer. Fisher's exact tests were used to compare the number of subjects with events on Sildenafil as compared to placebo and to perform subgroup analyses stratified by gender. Results: A total of 119 adverse events were reported by 25 patients (89\%). 42 (41 mild, 1 moderate) were thought to have a possible or probable relationship to study drug. Of those, $29(69 \%)$ occurred on Sildenafil while $13(31 \%)$ occurred on placebo $(p=0.01)$. One serious adverse event occurred during the study but was not related to study drug. There were no reports of loss of vision, loss of hearing, or priapism. Headache and flushing were the most commonly reported side effects (see Table 1). No subjects withdrew from the study as a result of an adverse event. 
Table 1: Summary of Side Effects

\begin{tabular}{|c|c|c|c|c|c|}
\hline & \multicolumn{2}{|c|}{ Sildenafil } & \multicolumn{2}{|l|}{ Placebo } & \multirow[b]{2}{*}{ p-value } \\
\hline & $\mathbf{N}$ & $\%$ & $\mathbf{N}$ & $\%$ & \\
\hline Headache & 9 & 33.3 & 5 & 17.9 & NS \\
\hline Male & 2 of 18 & 11.1 & 2 of 18 & 11.1 & NS \\
\hline Female & 7 of 9 & 77.8 & 3 of 10 & 30.0 & $* 0.07$ \\
\hline Flushing & 5 & 18.5 & 0 & 0.0 & $* 0.023$ \\
\hline Male & 0 of 18 & 0.0 & 0 & 0.0 & $\mathrm{~N} / \mathrm{A}$ \\
\hline Female & 5 of 9 & 55.6 & 0 & 0.0 & $* 0.011$ \\
\hline Dizziness & 2 & 7.4 & 2 & 7.1 & NS \\
\hline Nausea/Vomiting & 2 & 7.4 & 0 & 0.0 & NS \\
\hline Abdominal Pain & 1 & 3.7 & 0 & 0.0 & NS \\
\hline Kidney Stone & 1 & 3.7 & 0 & 0.0 & NS \\
\hline Photosensitivity & 1 & 3.7 & 0 & 0.0 & NS \\
\hline Rash & 1 & 3.7 & 0 & 0.0 & NS \\
\hline Diarrhea & 0 & 0.0 & 1 & 3.6 & NS \\
\hline Hypotension & 0 & 0.0 & 1 & 3.6 & NS \\
\hline Muscle Pain & 0 & 0.0 & 1 & 3.6 & NS \\
\hline Tinnitus & 0 & 0.0 & 1 & 3.6 & NS \\
\hline Tremulous & 0 & 0.0 & 1 & 3.6 & NS \\
\hline
\end{tabular}

Conclusion: Significant side effects did not occur with Sildenafil. Mild symptoms of headache and flushing were more common on Sildenafil than placebo, a difference seen primarily among females. Other side effects were reported with equal frequency on Sildenafil and placebo. Sildenafil appears to be well tolerated over a six-week period in children and young adults late after the Fontan Operation.

\section{The Severity of Congenital Heart Defects affects the Development of Social and Communication Skills in Children: a Follow-up Study from Birth to 18 Months}

Ragnhild Eek Brandlistuen, Kim Stene-Larsen, Henrik Holmstrøm, Markus A. Landolt, Leif T. Eskedal, Margarete E. Vollrath

Norwegian Institute of Public Health, Department of Pediatrics, Rikshospitalet University Hospital and University of Oslo, Oslo, Norway and Department of Pediatrics, Sørlandet Hospital HF, Kristiansand, Norway and University Children's Hospital, Zurich, Switzerland

Background: To study the social and communication development from 6 to 18 months of age in children with different types of CHD compared to controls. Methods: We linked prospective data from the Norwegian Mother and Child Cohort Study, conducted by the Norwegian Institute of Public Health with a nationwide medical CHD registry, and identified 198 toddlers with CHD in a cohort of 47,692 toddlers. Three groups of CHD were distinguished: $\operatorname{mild} /$ moderate $(\mathrm{n}=122)$, severe $(\mathrm{n}=$ 54), and CHD with comorbidity $(\mathrm{n}=22)$. At child age 6 and 18 months, the children's mothers reported on social and communication development by the Mother and Child questionnaire. Results: After adjusting for confounders (i.e., birth weight) and social/communication impairment at 6 months of age the CHD groups were significantly different from the controls on both communication $(\mathrm{F}=8.72, \mathrm{p}=<0.0001)$ and social development $(\mathrm{F}=7.84, \mathrm{p}=<0.0001)$ at 18 months of age. The adjusted group mean differences in fractions of standard deviations (SD) between the group of CHD with comorbidity and the controls was large on both communication $(\mathrm{SD}=0.74, \mathrm{p}=0.001)$ and social impairment ( $\mathrm{SD}=0.95, \mathrm{p}=<0.001)$. Severe CHD was also different from the controls with higher scores on both social $(\mathrm{SD}=0.31, \mathrm{p}=0.03)$ and communication $(\mathrm{SD}=0.36, \mathrm{p}=0.01)$ impairment. The mild/ moderate group showed only a small difference from the control group on communication development $(\mathrm{SD}=0.24, \mathrm{p}=0.009)$. Conclusion: Children with CHD show more social and communication impairment compared to controls at the age of 18 months depending on the severity of the CHD. It is necessary to broaden the scope of inquiry from neurodevelopmental domains to social and communication domains especially for children with severe CHD and CHD with comorbidity.

\section{Feeding Intolerance Following Norwood (Stage 1) Surgical Palliation for Hypoplastic Left Heart Syndrome}

Beesan Agha, Catherine Hough-Telford, Charlotte Woods-Hill, Mark Ayers, Patricia Mullens, Jeffrey Phillip Jacobs, Paul Jubeong Chai, James Anthony Quintessenza, David Cooper, Gul Dadlani

\section{All Children's Hospital, The Congenital Heart Institute of Florida (CHIF), and University of South Florida, Saint Petersburg, Florida}

Background: Feeding intolerance commonly affects infants with complex congenital heart disease. The causes for feeding problems have been attributed to the following: gastroesophageal reflux, congenital brain malformations, hypotonia, prolonged intubation, vocal cord dysfunction, delayed maternal bonding, and increased metabolic demands secondary to congestive heart failure. Hypoplastic left heart syndrome (HLHS) is a severe form of congenital heart disease that has been associated with feeding intolerance. Survival at our institution has previously been reported as follows (in patients without significant noncardiac abnormality or syndrome or 
prematurity less than 35 weeks or mechanical circulatory support prior to Stage 1): $86 \%$ at 30 days after stage 1 Norwood; $80 \%$ at hospital discharge; and $69 \%$ at one year after stage 1 Norwood procedure (Jacobs et al. Ann Thorac Surg 2008;85:1691-7). Inter-stage mortality and morbidity may be significantly affected by feeding intolerance. As part of a larger feeding initiative at our institution, we performed a retrospective review of feeding problems in patients with hypoplastic left heart syndrome who survived surgical palliation with a Norwood procedure. Methods: Retrospective review of HLHS patients surviving surgical Norwood (Stage 1) palliation between 2004 and 2008 was performed. The CardioAccess database was utilized for surgical data collection. Retrospective review of the electronic medical record was used for review of oral pharyngeal motility studies (OPMS), timing and necessity of postoperative gastrostomy tube placement, and feeding status at the time of discharge. All data were analyzed with percentages. Results: Twenty-four patients met inclusion criteria. $13 / 24(54 \%)$ patients had an OPMS prior to discharge: $1 / 13(7.5 \%)$ normal; $11 / 13(85 \%)$ partial failure (aspiration of some but not all consistencies); and $1 / 13(7.5 \%)$ complete failure (aspiration of all consistencies). Gastrostomy (G-tube) or gastrostromy-jejunal tube placement (G-J tube) was required in $17 / 24(71 \%)$ patients. Number of post-operative days from the Norwood procedure to G-tube placement ranged from the following: $2 / 17(12 \%)$ patients had G-tube placement $<30$ days after the Norwood; $12 / 17(70 \%)$ at 30-60 days; $2 / 17(12 \%)$ at $61-90$ days; $1 / 17(6 \%)$ at $>90$ days. At the time of discharge $7 / 24$ (29\%) patients were only orally fed; $12 / 24$ (50\%) were fed only via G-tube; and 5/24 (21\%) had a combination of G-tube and oral feeds. Hospital duration was also assessed based on the type of discharge feeding modality: infants discharged on oral feeds only had an average length of stay of 36.2 days (range 14-52 days); G-tube fed patients had an average length of stay of 86.8 days (range 44-188 days); combination feeders with G-tube and oral feeds had an average length of stay of 80.8 days (range 46-140 days). Conclusions: Feeding intolerance is common after a Norwood (Stage 1) procedure for hypoplastic left heart syndrome with $<30 \%$ of our patient population tolerating full oral feeds at the time of discharge. Many patients will need feeding assistance after a Norwood (Stage 1) procedure. In our program, we do not use home nasogastric feedings and instead place a gastrostomy tube when feeding assistance is necessary. Feeding intolerance increases the hospital length of stay in patients with hypoplastic left heart syndrome. Comprehensive pre- and post-operative feeding programs utilizing physicians, nurses, speech therapists, and nutritionists may significantly improve feeding intolerance and outcomes in patients with complex congenital heart disease in the future.

\section{Hearing Screening Following Norwood (Stage 1) Surgical Palliation for Hypoplastic Left Heart Syndrome}

Charlotte Woods-Hill, Catherine Hough-Telford, Beesan Agha, Emilio Quezada, Jeff Jeffrey Phillip Jacobs, Paul Jubeong Chai, James Anthony Quintessenza, David Cooper, Gul Dadlani

\section{All Children's Hospital, The Congenital Heart Institute of Florida (CHIF), and University of South Florida, Saint Petersburg, Florida}

Background: Auditory dysfunction can occur in infants with complex congenital heart disease. In these infants, auditory dysfunction can be attributed to a congenital defect in the auditory conduction system, associated neurocognitive deficits, injury to the central nervous system, extracorporeal membrane oxygenation (ECMO), cardiopulmonary bypass, or medications (such as diuretic therapy). Hypoplastic left heart syndrome (HLHS) is a severe form of congenital heart disease that has been associated with auditory dysfunction. At our institution, hearing exams are preformed in all infants prior to discharge. We performed a retrospective review of auditory problems in patients with hypoplastic left heart syndrome who survived surgical palliation with a Norwood (Stage 1) procedure. Methods: Retrospective review of patients surviving surgical Norwood (Stage 1) palliation for hypoplastic left heart syndrome between 2004 and 2008 were reviewed. The CardioAccess database was utilized for surgical data collection. Retrospective review of the electronic medical record was utilized for review of the hearing exams. All data was analyzed with percentages. Results: Twenty four patients met the inclusion criteria. Hearing exams demonstrated: normal exams in $21 / 24$ patients (88\%), unilateral hearing loss in $0 / 24$ patients and bilateral hearing loss in 3/24 (12\%) patients. Associated co-morbidities were also reviewed in those patients with normal hearing exams versus abnormal hearing exams. The use of ECMO occurred in 1/3 (33\%) abnormal hearing patients compared to $4 / 21$ (19\%) of the normal hearing patients. Seizures occurred in $0 / 3$ abnormal hearing patients and 5/21 (24\%) with normal hearing. Cerebral vascular accidents were noted in $1 / 3$ abnormal hearing patients and 4/21 $(19 \%)$ of the normal hearing patients. Abnormal 
chromosomes or associated congenital defects were noted in $1 / 3$ abnormal hearing patients and $2 / 21$ $(10 \%)$ of the normal hearing patients. The length of stay from Norwood (Stage 1) procedure to discharge was on average 92 days (range 33-138 days) for the abnormal hearing group compared to an average of 67.6 days (range 13-187 days) for the normal hearing group. Conclusions: Auditory dysfunction may occur after a stage 1 Norwood (Stage 1) procedure for hypoplastic left heart syndrome. Auditory dysfunction may cause significant life-long morbidity in this patient population and confound the diagnosis of learning disabilities and developmental delay in later life. Auditory dysfunction may occur in the more critically ill patients with hypoplastic left heart syndrome as identified by the increased length of stay. The impact of early detection and intervention in this patient population remains uncertain.

\section{Donor Troponin I Levels and Graft Survival in Pediatric Heart Transplantation}

Kimberly Lin, Patrick Sullivan, Abdul Salam, Beth Kaufman, Stephen Paridon, Brian D. Hanna, Thomas L. Spray, Janice Weber, Robert E. Shaddy

The Cardiac Center, The Children's Hospital of Philadelphia

Background: Troponin I (TnI), a biomarker that reflects myocardial damage in patients with acute coronary syndromes, is often obtained during evaluation of a potential transplant donor heart. It is unclear whether any elevation in donor TnI levels can predict an adverse outcome and should thus preclude acceptance of a donor heart. Methods: Deidentified data on heart transplants performed in recipients $<21$ years old between 4/07-4/09 was provided by the Organ Procurement and Transplantation Network. Donor TnI level and recipient survival without retransplantation (graft survival) were examined for statistical correlation. Other donor and recipient variables were examined as possible predictors of graft failure. Results: Overall graft survival in the 846 heart transplants performed was $81.1 \%$ at 2 years. 662 donors had at least one TnI level recorded prior to transplant, with values ranging from $0-50 \mathrm{ng} / \mathrm{ml}$ (median 0.1). Using the last donor TnI level reported prior to graft harvest, there was no correlation between TnI level and graft failure $(\mathrm{p}=0.635)$. An ROC curve suggested no association between $\mathrm{TnI}$ and graft status (AUC $=0.52 ; \mathrm{p}=0.59)$, and there was no difference in graft survival $(\mathrm{p}=0.519)$ among quartiles of of $\mathrm{TnI}$ $(<0.04, \quad 0.04-0.1, \quad 0.1-0.35, \quad>0.35 \mathrm{ng} / \mathrm{ml}) . \quad 72$ transplanted donors had a $\mathrm{TnI}>1$; graft survival was not associated with $\mathrm{TnI}>1(78 \%)$ vs. TnI $<1(82 \%)$ at 2 years $(\mathrm{p}=0.961)$. TnI values were also not associated with post-transplant hospital length of stay $(r=-0.035 ; p=0.372)$. Conclusion: In donor hearts accepted for pediatric heart transplantation, pre-harvest TnI elevations are not associated with increased graft failure. Further prospective studies are needed to help determine whether a threshold value for elevated troponin in heart transplantation exists.

This work was supported in part by Health Resources and Services Administration contract 2342005-370011C. The content is the responsibility of the authors alone and does not necessarily reflect the views or policies of the Department of Health and Human Services, nor does mention of trade names, commercial products, or organizations imply endorsement by the U.S. Government. 